\title{
A Priority Rate-Based Routing Protocol For Wireless Multimedia Sensor Networks
}

Loini Nakadhilu Methimbo Tshiningayamwe

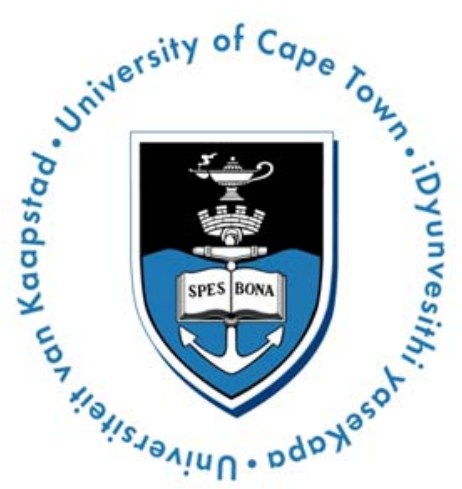

This thesis is submitted in complete fulfillment of the academic requirements for the degree of

Master of Science in Electrical Engineering in the Faculty of Engineering and The Built Environment

University of Cape Town 
The copyright of this thesis vests in the author. No quotation from it or information derived from it is to be published without full acknowledgement of the source. The thesis is to be used for private study or noncommercial research purposes only.

Published by the University of Cape Town (UCT) in terms of the non-exclusive license granted to UCT by the author. 
As the candidate's supervisor, I have approved this dissertation for submission.

Name: Dr. Mqhele E. Dlodlo, Associate Professor

Signed: Signed by candidate

Date: 2017-06-19 


\section{Declaration}

I declare that this thesis is my own work. Where collaboration with other people has taken place, or material generated by other researchers is included, the parties and/or materials are indicated in the acknowledgements or are explicitly stated with references as appropriate.

This work is being submitted for the Master of Science in Electrical Engineering at the University of Cape Town. It has not been submitted to any other university for any other degree or examination.

25 August 2016

Loini Tshiningayamwe

Date 


\section{Dedication}

To God Almighty.

To my parents Jacobina and Tomas Tshiningayamwe. 


\section{Abstract}

The development of affordable hardware has made it possible to transmit multimedia data over a wireless medium using sensor devices. Deployed sensors span larger geographical areas, generating different kinds of traffic that need to be communicated either in real-time or non-realtime mode to the sink. The tiny sized design of sensor nodes has made them even more attractive in various environments as they can be left unattended for longer periods.

Since sensor nodes are equipped with limited resources, newer energy-efficient protocols and architectures are required in order to meet requirements within their limited capabilities when dealing with multimedia data. This is because multimedia applications are characterized by strict quality of service requirements that distinctively differentiate them from other data types during transmission. However, the large volume of data produced by the sensor nodes can easily cause traffic congestion making it difficult to meet these requirements. Congestion has negative impacts on the data transmitted as well as the sensor network at large. Failure to control congestion will affect the quality of multimedia data received at the sink and further shorten the system lifetime.

Next generation wireless sensor networks are predicted to deploy a different model where service is allocated to multimedia while bearing congestion in mind. Applying traditional wireless sensor routing algorithms to wireless multimedia sensor networks may lead to high delay and poor visual quality for multimedia applications.

In this research, a Priority Rate-Based Routing Protocol (PRRP) that assigns priorities to traffic depending on their service requirements is proposed. PRRP detects congestion by using adaptive random early detection (A-RED) and a priority rate-based adjustment technique to control congestion.

We study the performance of our proposed multi-path routing algorithm for real-time traffic when mixed with three non real-time traffic each with a different priority: high, medium or low. Simulation results show that the proposed algorithm performs better when compared to two existing algorithms, PCCP and PBRC-SD, in terms of queueing delay, packet loss and throughput. 


\section{Acknowledgements}

I would like to express my sincere gratitude to God Almighty for His endless love and guidance throughout my studies. Lord, may your kind grace continue holding me close to you always.

To my supervisor Prof Mqhele Dlodlo and my co-supervisor Dr Guy Alain Lusilao-Zodi, words are not enough to express how grateful I am for having you as my supervisors. Your words of encouragement and academic support are truly appreciated; thank you very much for always pushing me and having faith in me.

To my MED Communication Research group, your support is very much appreciated.

Special thanks go to my wonderful husband David SI Iiyambo. Your unconditional love and understanding during my studies are highly appreciated. You will forever be truly cherished.

My dearest mom Jacobina Tshiningayamwe and dad Tomas Tshiningayamwe, may God's light continue shining on you for having been the wonderful parents you are and for always keeping me in your prayers. Lastly, to all my very dear siblings, I cannot wish for any better siblings, you guys are simply the best. Your continuous support and encouragement will never cease to amaze me. Please continue being the beautiful and wonderful pillars of strength that you are. 


\section{Table of Contents}

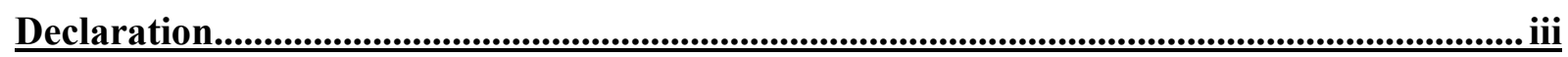

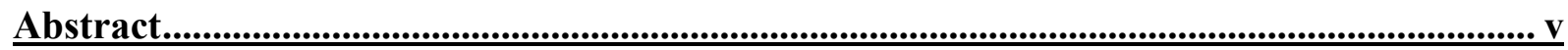

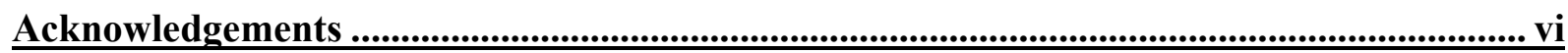

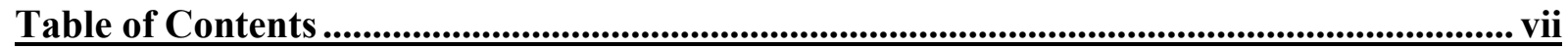

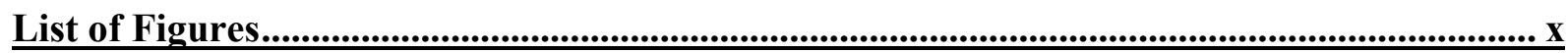

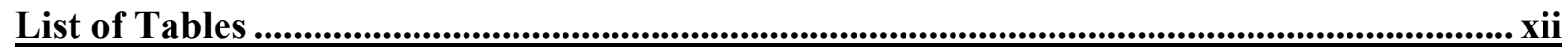

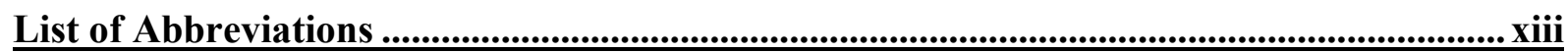

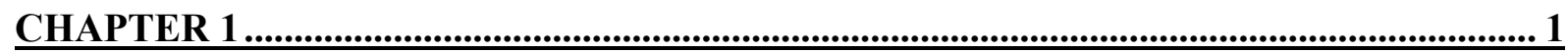

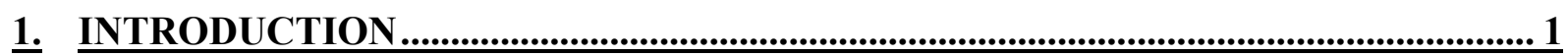

1.1 Overview of Wireless Multimedia Sensor Networks .............................................................. 1

1.1.1 Overview of WMSNs requirements..............................................................................................

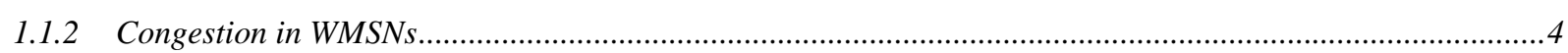

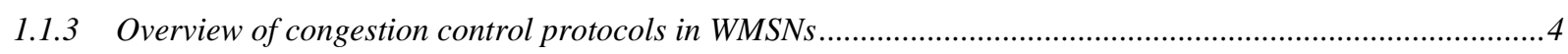

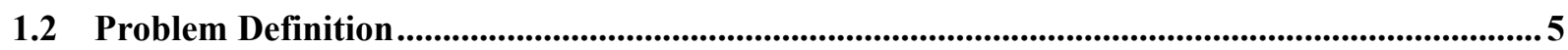

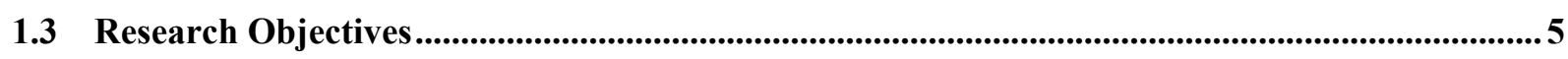

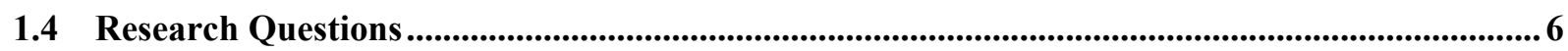

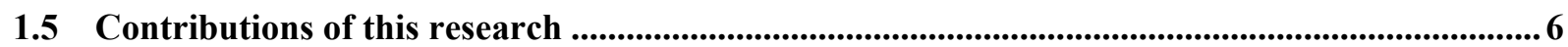

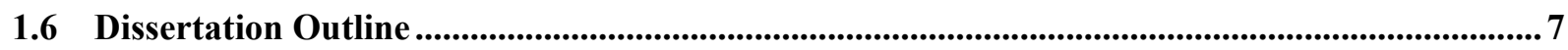

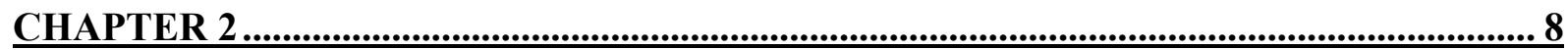

2. CONGESTION CONTROL IN WIRELESS MULTIMEDIA SENSOR NETWORKS 8

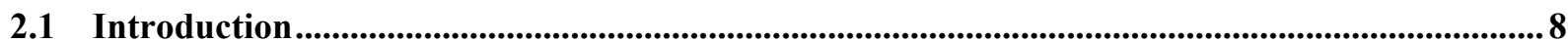

2.2 Requirements and Challenges of WMSNs.................................................................................... 8

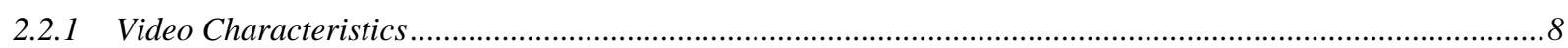

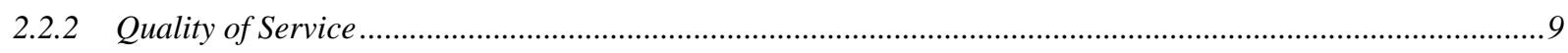

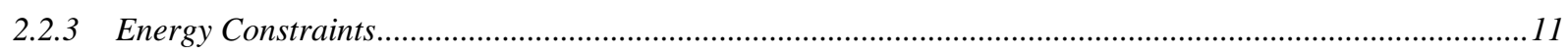

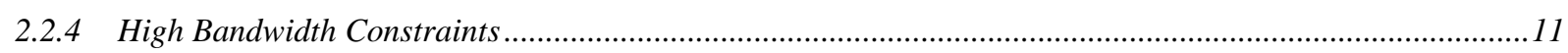

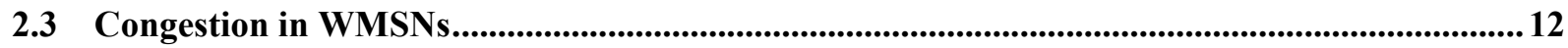

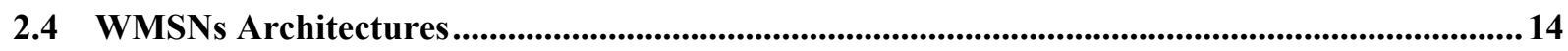

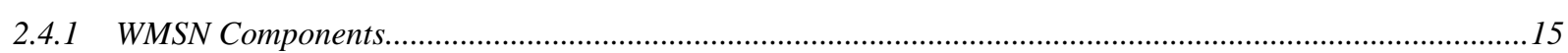

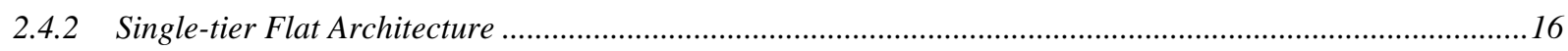




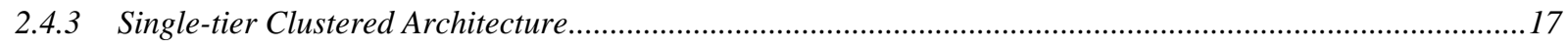

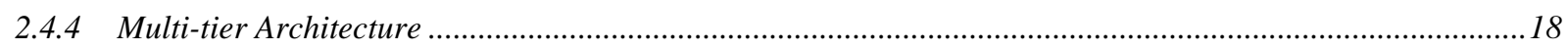

2.5 Congestion control protocols in WMSNs ..............................................................................20

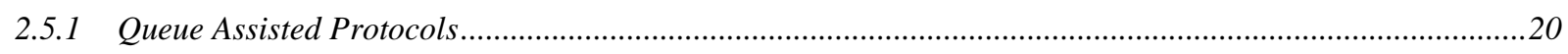

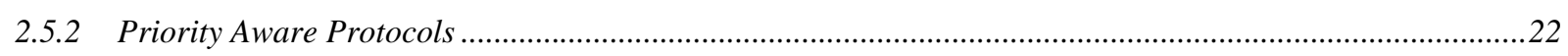

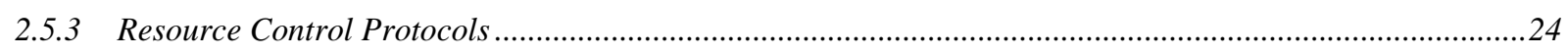

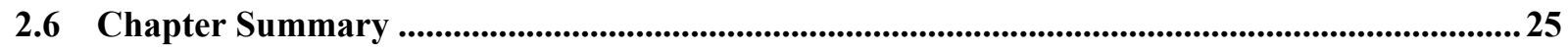

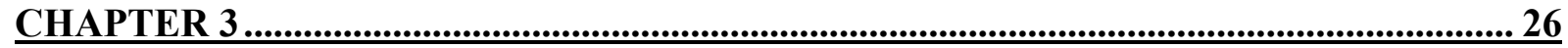

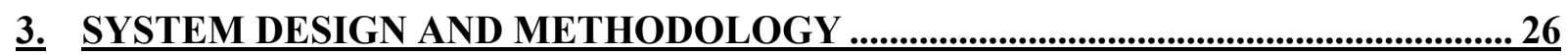

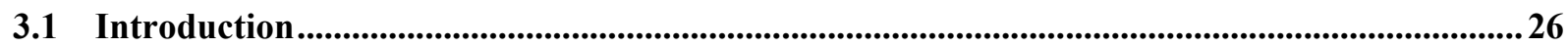

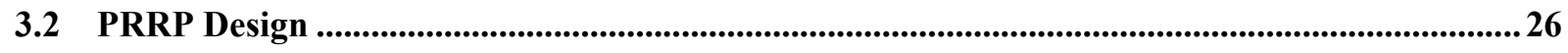

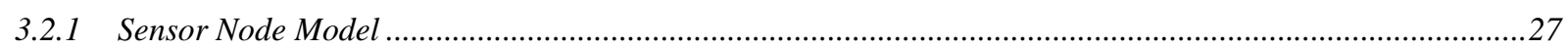

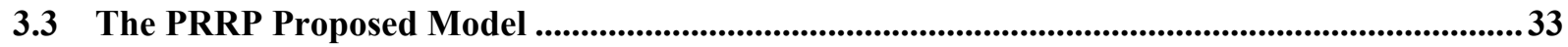

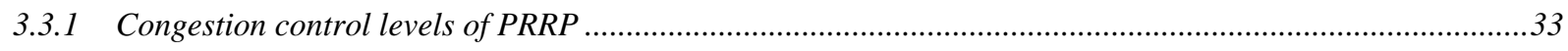

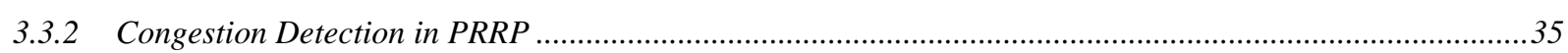

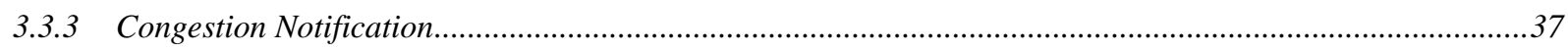

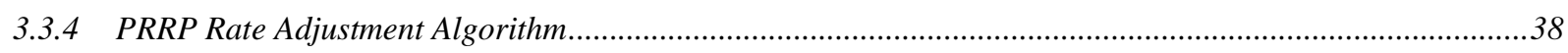

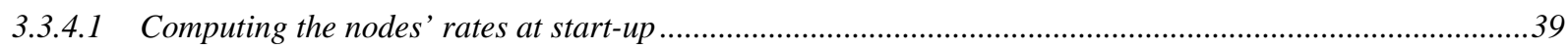

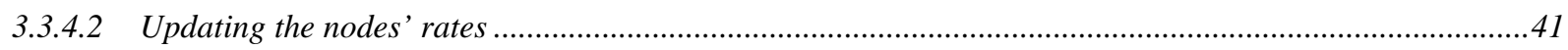

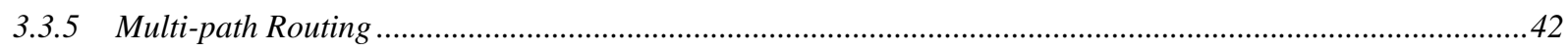

3.4 Chapter Summary ................................................................................................................44

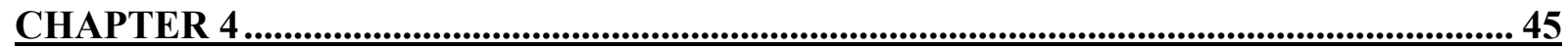

4. SIMULATION APPROACH, RESULTS AND DISCUSSION................................45

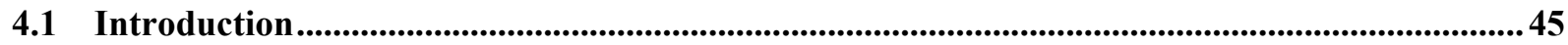

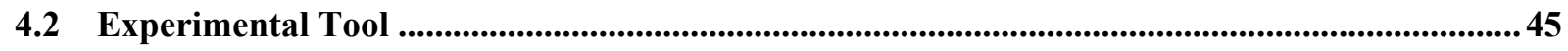

4.3 Performance Evaluation Metrics................................................................................46

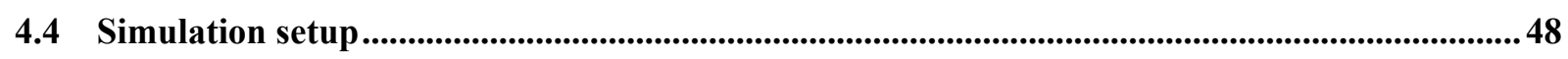

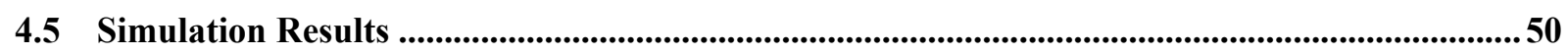

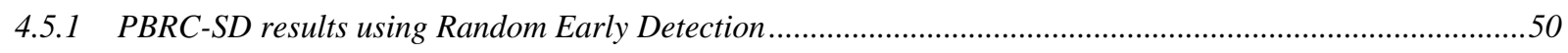

4.5.2 PRRP results using Adaptive Random Early Detection .................................................................................61

4.5.3 Performance of the Service Differentiation unit..................................................................................66

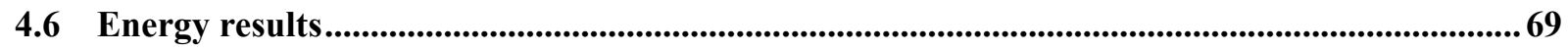

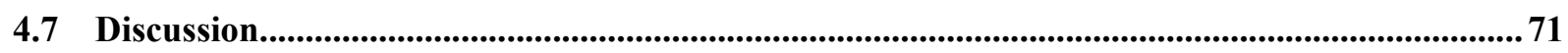

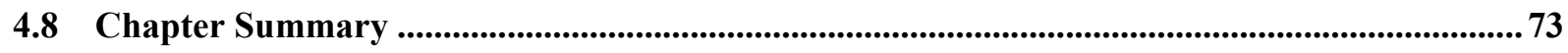




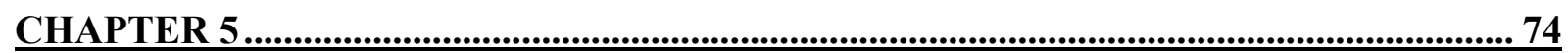

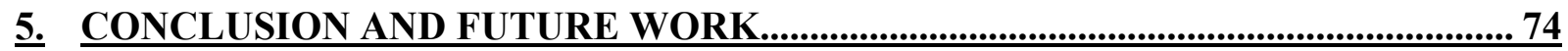

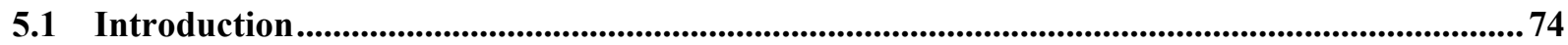

5.2 Summary of this research............................................................................................................. 74

5.3 Recommendations for Future work ..................................................................................................... 75

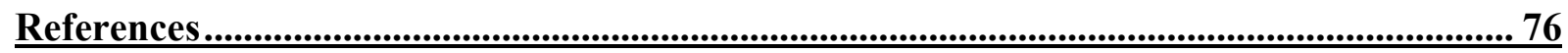

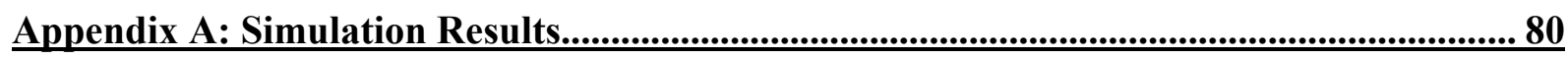

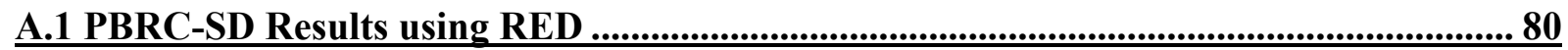

Constant Bit Rate traffic and Fixed service time ...............................................................................

Exponential traffic and Random service time .................................................................................................8 80

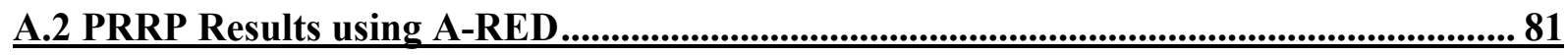

Exponential traffic and Random Service time results .................................................................................... 81 


\section{List of Figures}

Figure 1.1: Components of a wireless sensor node....................................................... 1

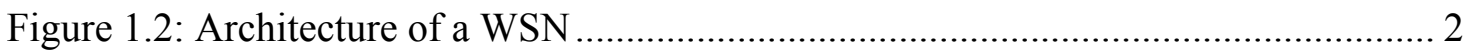

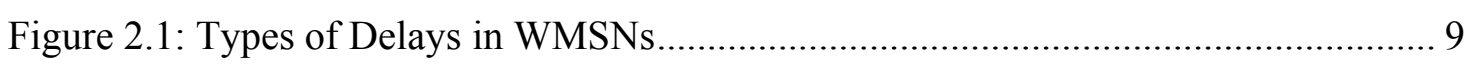

Figure 2.2: Link level and node level congestion .................................................... 13

Figure 2.3: WMSN Architecture .............................................................................. 14

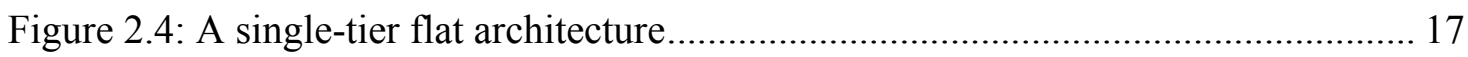

Figure 2.5: Single-tier clustered Architecture........................................................ 18

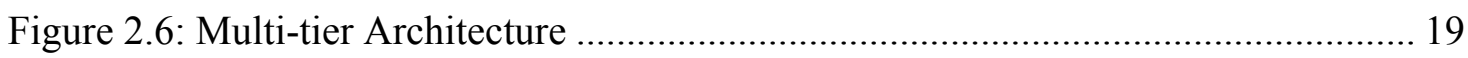

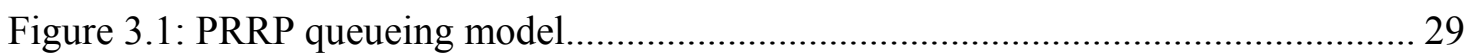

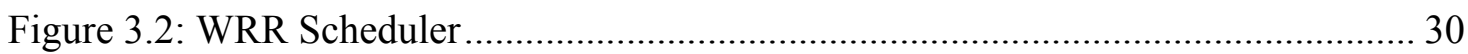

Figure 3.3: Single-path Routing with extra parent node as considered in PRRP ............. 31

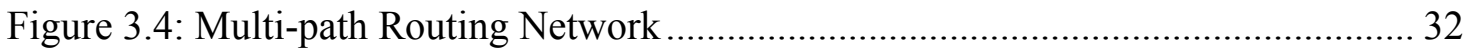

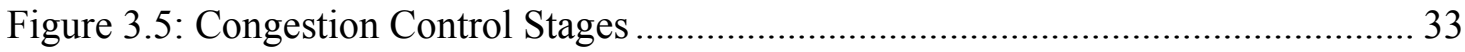

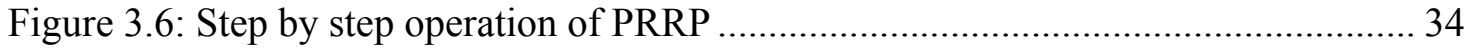

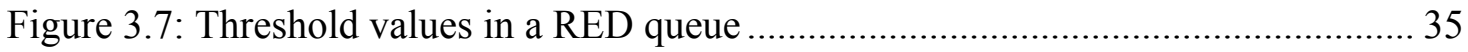

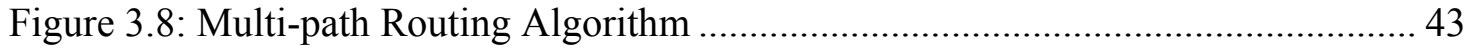

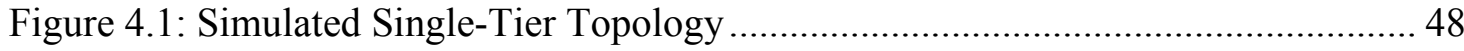

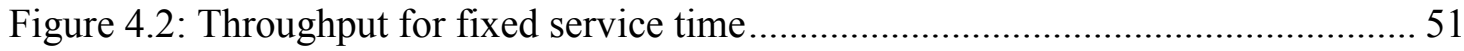

Figure 4.3: Loss probability and normalized throughput for random service time .......... 53

Figure 4.4: Normalized throughput and loss probability for fixed service time............... 55

Figure 4.5: Normalized throughput and loss probability for random service time.......... 57

Figure 4.6: Normalized throughput for fixed service time ........................................ 59

Figure 4.7: Normalized throughput and loss probability for random service time........... 60 
Figure 4.8: Normalized throughput and loss probability of random service time 62

Figure 4.9: Normalized throughput and loss probability for random service time. 64

Figure 4.10: Results for random service time 65

Figure 4.11: Class based results for PBRC-SD 67

Figure 4.12 Normalized throughput and delay (s) per class for PCCP and PRRP 69

Figure 4.13 Energy consumption 70

Figure 4.14 Residual Energy. 71 


\section{List of Tables}

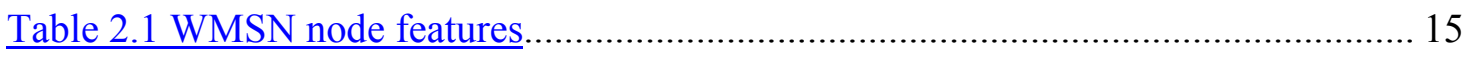

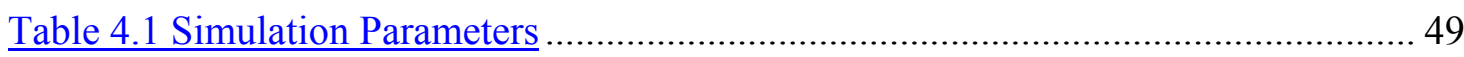

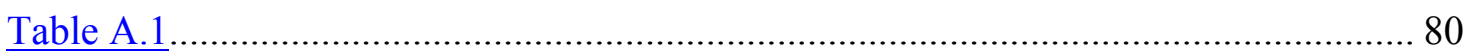

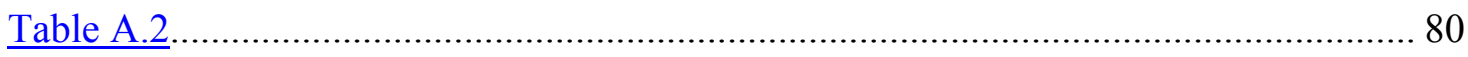

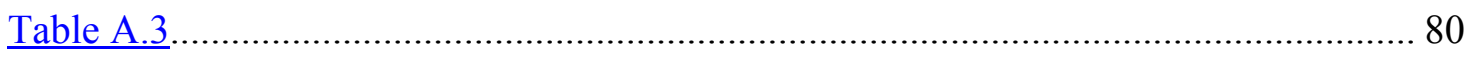

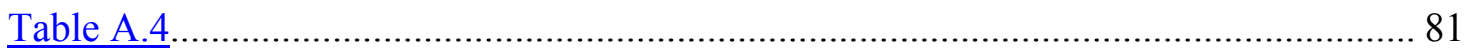

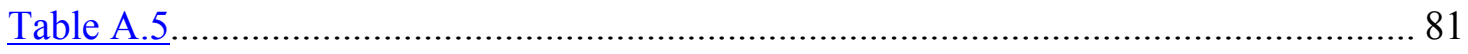

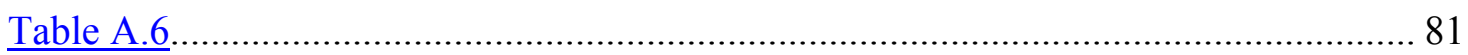

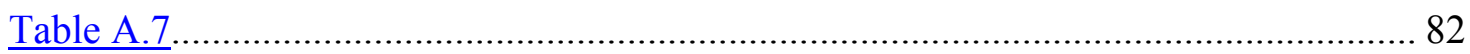

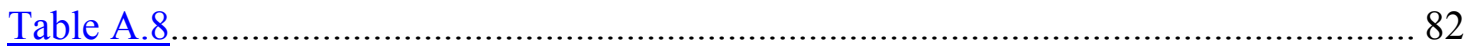




\section{List of Abbreviations}

$\begin{array}{ll}\text { AIMD } & \text { Additive Increase Multiplicative Decrease } \\ \text { A-RED } & \text { Adaptive Random Early Detection } \\ \text { BER } & \text { Bit Error Rates } \\ \text { CBR } & \text { Constant Bit Rate } \\ \text { CCF } & \text { Congestion Control and Fairness } \\ \text { CMOS } & \text { Complementary metal oxide } \\ \text { CODA } & \text { Congestion Detection and Avoidance } \\ \text { DCT } & \text { Discrete Cosine Transform } \\ \text { DPCM } & \text { Differential Pulse-Code Modulation } \\ \text { ETM } & \text { Exponential Traffic Model } \\ \text { EWMA } & \text { Exponential Weighted Moving Average } \\ \text { EXPO } & \text { Exponential } \\ \text { FIFO } & \text { First in first out } \\ \text { GB } & \text { Gigabytes } \\ \text { HP-NRT } & \text { High Priority Non real-time traffic } \\ \text { HP-RT } & \text { High Priority Real-Time traffic } \\ \text { HTAP } & \text { Hierarchical Tree Alternative Path } \\ \text { ISM } & \text { Industrial, Scientific, and Medical } \\ \text { JPEG } & \text { Joint Photographic Experts Group } \\ \text { KAlobits per second }\end{array}$




\begin{tabular}{|c|c|}
\hline MAX & Maximum \\
\hline Mbps & Megabits per second \\
\hline MP-NRT & Medium Priority Non real-time traffic \\
\hline MEM & Micro Electro Mechanical system \\
\hline MIN & Minimum \\
\hline MPEG & Moving Picture Experts Group \\
\hline $\mathrm{MHz}$ & Megahertz \\
\hline NED & Network Description \\
\hline NS & Network Simulator Version \\
\hline OMnet & Objective Modular Network Testbed \\
\hline OPNET & Optimized Network Engineering Tools \\
\hline OTcl & Objected-Oriented Tool Command Language \\
\hline PBRC-SD & Priority-Based Rate Control for Service Differentiation \\
\hline PCCP & Priority-based congestion control protocol \\
\hline PRRP & Priority-Rate Based Routing Protocol \\
\hline QoS & Quality of Service \\
\hline RAM & Random Access Memory \\
\hline RCCP & Receiver Congestion Control Protocol \\
\hline RED & Random Early Detection \\
\hline ROM & Read-Only Memory \\
\hline SCAP & Source Congestion Avoidance Protocol \\
\hline SNR & Signal-to-Noise Ratio \\
\hline ТCP & Transmission Control Protocol \\
\hline UDP & User Datagram Protocol \\
\hline
\end{tabular}


UWB Ultra-Wide Band

WRR Weighted Round Robin

WCCP Wireless Multimedia Sensor Network Congestion Control Protocol

WMSN Wireless Multimedia Sensor Network

WSN Wireless Sensor Network 


\section{CHAPTER 1}

\section{INTRODUCTION \\ 1.1 Overview of Wireless Multimedia Sensor Networks}

Recent advances in Micro Electro Mechanical Systems (MEMs), wireless technologies, complementary metal oxide (CMOS) cameras, microphones and sensors have led to the development of Wireless Sensor Networks (WSNs) and Wireless Multimedia Sensor Networks (WMSNs) [1] [2].

Sensors found in WSNs and WMSNs are tiny devices used to collect data such as light, pressure, vibrations and temperature from the physical environment and convert this data into meaningful information [3]. Sensors have seen many people anticipating a revolution in our day-to-day living as they are expected to be laid under roads, walls, soil and machines that bridge between the physical world and the virtual world. Normally, sensors are interfaced with co-operative devices called nodes that are equipped with computing capabilities, thus the name sensor nodes. These sensor nodes are low-cost devices, equipped with limited resources such as power, processing, communication and storing capabilities. A sensor device consists of five key components: a sensor, memory, processor, power and transceiver. Optional components such as the geographical positioning system and the mobiliser are added depending on the application of the sensor node. Figure 1.1 shows how the different components of a sensor node are connected.

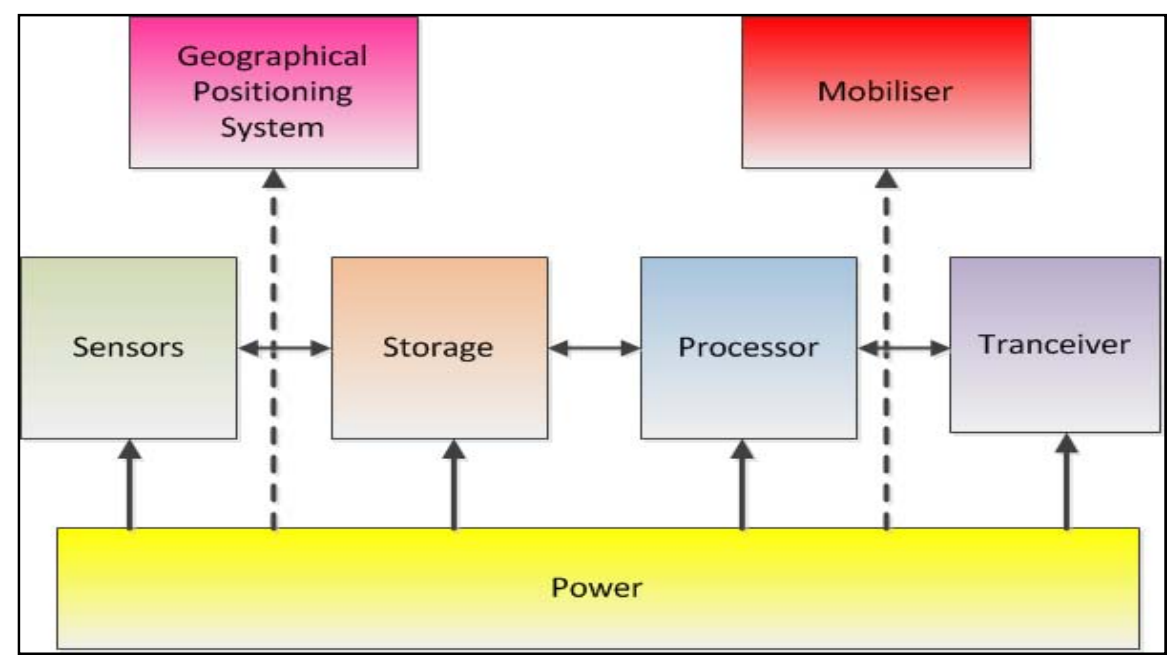

Figure 1.1: Components of a wireless sensor node 
Normally, sensors are specifically designed to carry out application specific tasks therefore they range from simple sensors to more advanced sensors. Common examples of sensors are: chemical sensors, mechanical sensors, thermal sensors, biological sensors, optical sensors and magnetic sensors [1]. Data collected by these sensors are saved in the memory unit, which consists of Random Access Memory (RAM) and Read Only Memory (ROM). Since many different sensors can be used to gather data, they can easily generate copies of the same physical parameters. Therefore, the processor applies data compression and aggregation techniques to remove redundancies in the data sent to the sink. Sensors use transceivers to communicate the data to their directly connected nodes. The frequency ranges supported by sensor devices are $400 \mathrm{MHz}, 800-900 \mathrm{MHz}$, and $2.4 \mathrm{GHz}$ - the Industrial, Scientific, and Medical (ISM) respectively. The sensors can use AA batteries which store $2.2-2.5$ Ah at 1.5 $\mathrm{V}$ to provide them with power that is required by the sensor device, memory unit, processor and transceiver [4].

A collection of sensor nodes is connected through wireless links to form a Wireless Sensor Network (WSN). A WSN often consists of thousands of interconnected sensors which generate scalar data, process it and communicate the data to a base station, which is also known as the sink. Figure 1.2 depicts a WSN.

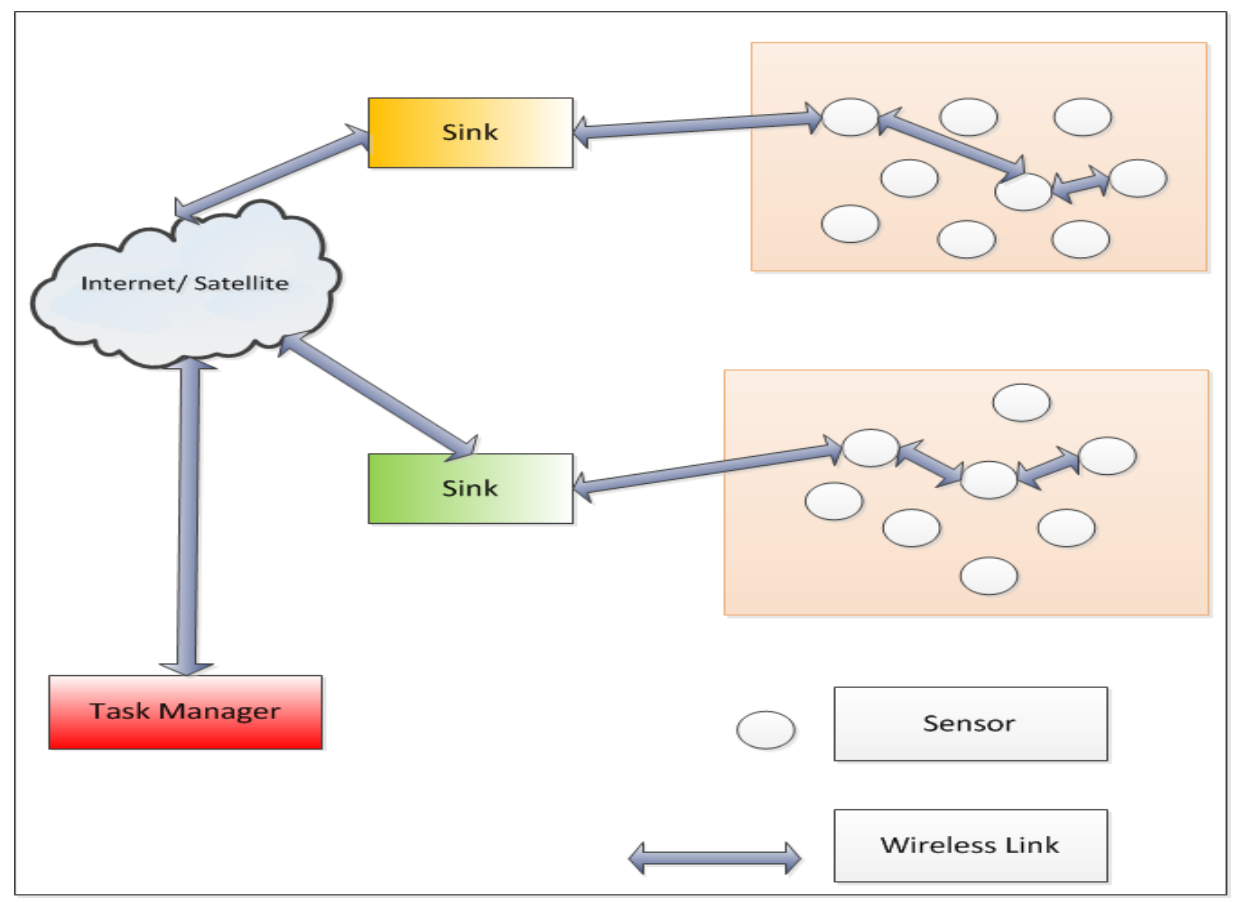

Figure 1.2: Architecture of a WSN 
The need to collect delay-sensitive data has led WSNs to expand into another technology called Wireless Multimedia Sensor Networks (WMSNs) that transfer videos, texts, graphics, audio and images over wireless communication media [5]. To achieve this, sensors are equipped with cameras and microphones which enable them to generate multimedia data and scalar data and send it to a base station. The traffic generated might be of different levels of importance, therefore WMSNs are required to support different traffic classes to satisfy the different service requirements thereof [6]. The network is therefore required to ensure that it provides more resources to the most important traffic as opposed to the least important traffic.

WSNs and WMSNs have diverse applications ranging from military, industries, home, hospitals, transportation, agriculture, crime surveillance and road traffic management. These networks are usually deployed in a self-organising or deterministic manner depending on the application. When compared with traditional networks, WSN provides a number of benefits such as low cost, scalability, reliability, accuracy and flexibility.

Ensuring efficiency in WMSNs remains a challenge as the network paradigm needs to be constantly redesigned to obtain new efforts that can meet the requirements of WMSNs. The majority of such efforts are concerned with techniques that can meet the requirements of WMSNs as discussed in [7] [8] [5].

\subsubsection{Overview of WMSNs requirements}

WMSNs applications deliver large traffic volumes to the sink either in real-time or nonreal-time mode. These large traffic volumes consist of different characteristics making it difficult for the resource-constrained sensors to meet the WMSNs' application requirements. Furthermore, data redundancies occur as sensor nodes also collect traffic from their neighbouring sensors' range thus contributing to the high traffic volume. Nodes may constantly enter and leave the network due to various reasons and this may impact the routing paths used to transmit data.

The characteristics discussed above introduce unique requirements and challenges for WMSNs, such as high bandwidth demand, limited energy, application specific Quality of Service (QoS) requirements [3], limited processing and storage capabilities and short communication range [4].

These requirements are difficult to meet due to the presence of congestion caused by the high traffic volumes produced by real-time and non real-time data. 


\subsubsection{Congestion in WMSNs}

In communication networks, congestion is defined in several ways. Tanenbaum [9] defines congestion as the presence of too many packets in the network causing the network's performance to slow down due to insufficient network capacity. Another definition provided by Leon-Garcia and Widjaja [10] states that congestion is the build-up of packets at a buffer that occur when the output rate is greater than the input rate. This causes the buffer to reject all the incoming packets due to the lack of buffer space. Both definitions are acceptable in defining congestion in WMSNs.

Due to the upstream manner in which heterogeneous traffic is sent, congestion can easily occur depending on the delivery model used and the resources available at the nodes. Congestion has negative impacts on the audio and video streams, including high delay, high jitter, packet loss and low throughput [11] [12]. It is therefore important to design and implement efficient congestion control mechanisms that can reduce the effect of congestion on multimedia data over a WSN.

Motivated by the effects of congestion on WMSNs, the purpose of this research is to implement a congestion control protocol for WMSNs that consider the priority of the multimedia traffic when allocating transmission rates to the nodes. Certain WMSN applications need to send urgent data to the sink which needs to reach the sink without any delays, packet loss or degradation in the visual and audio quality of the received images, audio and video streams, thus the importance of the priority rate-based congestion control protocol.

\subsubsection{Overview of congestion control protocols in WMSNs}

Several congestion control protocols $[13][14][15][16]$ have been proposed in the last decade which reduce the effects of congestion in WMSNs. They can be classified as traffic control protocols, resource control protocols and a hybrid of both protocols. Traffic control protocols operate by monitoring the number of packets injected in the network. Too many packets use up the limited bandwidth capacity available at the nodes, putting the applications' quality of service (QoS) at stake. Traffic control protocols therefore alleviate congestion by reducing or increasing the amount of traffic in the network depending on the network status. On the other hand, resource control protocols are concerned with adding more resources to the network during congestion. This mechanism is expensive to implement and can waste resources. The hybrid congestion control mechanism is a combination of traffic control and 
resource control techniques. The choice on which protocol to opt for depends on the requirements of the application.

\subsection{Problem Definition}

Wireless multimedia sensor networks deal with different applications with varying levels of service requirements. For real-time traffic over WMSNs, it is important when routing the traffic to integrate as parameters their stringent packet delay, jitter and high bandwidth requirements to meet their application services requirements.

Moreover, due to high bandwidth demand of multimedia traffic, congestion may easily occur in low-resource bandwidth networks such as a wireless multimedia sensor networks. In wireless multimedia sensor networks, congestion can waste scarce resources such as the energy of nodes, and affect application specific QoS requirements, which can easily result in poor visual and sound quality of the transmitted images, audio and video.

When the nodes are congested in WMSNs, proper rate adjustment can be performed to reduce congestion and to guarantee efficient link utilization. Rate adjustment techniques change the exact rates at which sensor nodes should transmit data by looking at either the service rate, the scheduling rates or the source output rates.

Therefore, considering the requirements of multimedia applications, the limitations of the WSNs and the effects of congestion on the performance of wireless multimedia sensor networks, this research aims to address the issues mentioned above by implementing an energy efficient priority rate-based congestion control protocol which assigns priority-dependent rates to traffic.

\subsection{Research Objectives}

The preceding sections clearly motivate the need of congestion control in WMSNs. Therefore, the main objective of this research is to design a priority-rate based routing protocol for service differentiation in wireless multimedia sensor networks. Some of the characteristics of the proposed protocol are the improvement of the lifetime of the sensor network by considering the energy of upstream nodes, and the provision of services to multimedia traffic based on the application requirements.

Therefore, the proposed priority rate-based congestion control protocol is aimed at: 
1. Organising multimedia traffic and scalar data into different classes based on their service requirements and allocating a priority based rate to traffic classes.

2. Prioritising real-time traffic while being fair to non real-time traffic.

3. Supporting multi-path routing by considering the node energy levels when transmitting traffic into the network.

\subsection{Research Questions}

The main research question posed is formulated as follows:

"How should the wireless multimedia sensor network allocate transmission rates to traffic with different service requirements so as to meet the needs of each traffic class and the wireless sensor network's energy constraints?"

The main research question can thus further be unpacked into the following three subquestions:

1. How should a wireless sensor network organise traffic and allocate transmission rates to meet the strict service requirements of multimedia traffic?

2. How should the wireless sensor network prioritise multimedia traffic while remaining fair to other competing traffic?

3. How can the adaptive rate protocol better preserve the energy of nodes in a multi-path WMSN?

\subsection{Contributions of this research}

Based on the objectives described above, this dissertation makes the following contributions in the field of research of congestion control in Wireless Multimedia Sensor Networks.

1. Congestion detection using Adaptive Random Early Detection (A-RED) threshold values: Various strategies in literature have been introduced to detect the presence of congestion in WMSNs. Among these strategies, Random Early Detection (RED) is the most utilised method. However, unlike RED which uses static values for determining the threshold values, A-RED uses dynamic values as threshold. Congestion control using A-RED values in WMSNs has never been done before.

2. Energy efficient congestion control protocol: The proposed congestion control protocol considers the energy levels of the upstream nodes before it can send traffic to them. This consideration is made due to the energy limitations in wireless sensor nodes. 
Checking the available energy of the sensor nodes before transmitting traffic to it will guarantee reliable delivery of real-time data at the sink and will further extend the lifetime of the sensor network.

3. Multi-path routing: A multi-path routing strategy is considered in the proposed protocol whereby more than one path exists between selected nodes in the WMSN. If a node wishes to send traffic to the sink, it will choose to send the traffic through the less congested nodes so as to avoid congestion.

These contributions have been accepted and published in Proceedings of the Springer Conference NABIC2015. The reference is:

1. L. Tshiningayamwe, G. Zodi and M. Dlodlo, "A Priority Rate-Based Routing Protocol for Wireless Multimedia Sensor Networks", in Nature and Biologically Inspired Computing, Pietermaritzburg, 2015, pp. pp 347-358.

\subsection{Dissertation Outline}

The rest of this study is organised as follows:

CHAPTER 2: CONGESTION CONTROL IN WMSNs: This chapter provides in-depth discussion on WMSNs' requirements and the causes and effects of congestion in WMSNs. Existing congestion control techniques, as well as existing WMSNs architectures, are further discussed

CHAPTER 3: SYSTEM DESIGN AND METHODOLOGY: The approach and methodology followed throughout the research process is presented. The design and implementation of the priority-based rate controller is discussed.

CHAPTER 4: SIMULATION APPROACH, RESULTS AND DISCUSSION: The simulation environment used to conduct the research is presented. Discussions include the performance metrics considered for the network analysis. The results obtained while evaluating the performance of the priority-based rate controller are discussed and compared with existing and well accepted protocols, in terms of throughput, delay and loss.

CHAPTER 5: CONCLUSION AND FUTURE WORK: The salient points of the research work are highlighted and conclusions drawn. In this chapter, some suggestions for future work are discussed. 


\section{CHAPTER 2}

\section{CONGESTION CONTROL IN WIRELESS MULTIMEDIA SENSOR NETWORKS}

\subsection{Introduction}

This chapter provides an in-depth discussion of the requirements of WMSNs which must be taken into consideration during the design and implementation of WMSN protocols and architectures. It further discusses congestion and its effects on multimedia traffic and the network resources. In order to reduce congestion in WMSNs, a number of congestion control protocols have been proposed. These protocols are also presented in this chapter.

\subsection{Requirements and Challenges of WMSNs}

As mentioned in Chapter 1, WMSNs consist of a mixture of multimedia data that need to reach the base station either in real-time or non real-time. Real-time data have strict requirements such as low delay, low jitter, low packet loss and high bandwidth demand [17]. The high bandwidth demand and low delay requirement pose further challenges to sensor nodes in terms of processing and energy capabilities required, for the application to function effectively. Meeting these requirements in low power and low processing networks such as wireless sensor networks is a challenging task.

Over the past few years, various approaches on how to provide services to multimedia content over WSN have been proposed in the literature [18][19][20]. The various solutions range from novel compression techniques for video, to new approaches of designing the network solution, to redefining the architecture of the WSN. However, meeting these solutions require an understanding of the challenges and requirements of WMSNs. Before diving into an in-depth discussion of the challenges of WMSN, it is important to discuss the characteristics of video and audio, which constitute multimedia data.

\subsubsection{Video Characteristics}

Multimedia data consist of snapshots and streaming multimedia content which is produced by heterogeneous sensors [6]. Streaming multimedia content takes several hours to 
transmit, while snapshots take shorter periods to transmit. This data produce bursty and high traffic volumes which impact the resources of the sensor nodes.

Despite the vast potential of multimedia communication in WSN, there exist numerous challenges which if not properly addressed will hinder the realization of such a revolutionary technology. These challenges are discussed in detail below.

\subsubsection{Quality of Service}

In computer networks, Quality of Service (QoS) is the capability of the network to provide data flow with resource assurance and appropriate service to meet its requirements. This means that every application in a network has certain expectations on the quality of service it should receive from the network. For real-time traffic, QoS is defined in terms of packet delay, loss and jitter.

a) Packet Delay: Real-time data have stringent delay requirements and traffic is expected to reach the sink within a specified time after it has been generated. Delay is the difference between the time a packet is sent from a source and the time it is received at the destination. Packet delay may occur as a result of propagation, queuing, processing and transmission of packets [21]. Figure 2.1 below illustrates the four types of delays experienced by traffic in WMSNs.

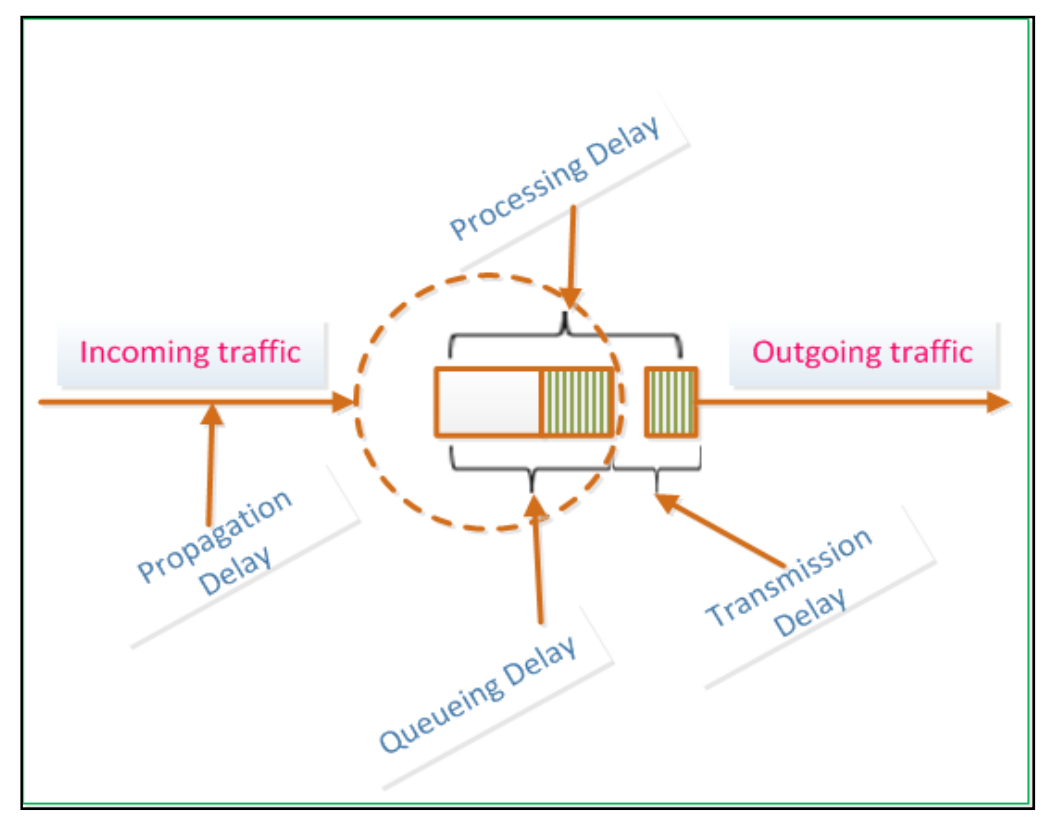

Figure 2.1: Types of Delays in WMSNs 
Propagation delay refers to the time it takes a bit to propagate through the transmission medium, which is determined by the speed of the link that attaches the source to the destination. Transmission delay is the time it takes to transmit a packet at a node. It depends on the length of the packet and the transmission rate of the link. The higher the transmission rate, the less time it takes to send the packet into the link. During transmission of packets, packets pass through a finite buffer at every intermediate node for processing. While in the queue, packets may experience queuing delay, especially when there were already packets queued prior to the arrival of the packet. The processing delay refers to the time it takes to determine the next route of the packet. Upon arrival of packets at each node, the nodes, especially if it is a parent node, may need to do data aggregation, which may cause some additional delays. It is important to control multimedia traffic because a small variation in the arrival of real-time packets at the destination will render information of poor quality, wasting the sensor nodes' resources.

b) Packet loss: In WMSNs, a packet is the smallest unit of measurement of the data acquired by sensors. The packet contains data of varying degrees of importance measured from different sensors in the network. Packet loss can occur as a result of congestion and changes in network topology [22]. In the event a packet is dropped, retransmitting such a packet can deplete the energy at the nodes thus making packet loss undesirable in WMSNs. Some real-time applications tolerate a small amount of packet loss, usually between $1 \%$ to $10 \%$ of the total traffic. Similar to packet delay, packet loss will affect the overall quality of the multimedia data and will waste the WMSN resources.

c) Jitter: Jitter refers to the variation in delay between consecutive packets. For example, for a streaming session over a WSN, a video clip may take up an average delay of 20 seconds for packets to be delivered at the destination. If there is variation between two consecutive packets, with the first packet arriving after 20 seconds and the other after 30 seconds, this means that the jitter is equal to 10 seconds. High jitter may affect the visual quality of the video clip or interrupt a live conversation. 


\subsubsection{Energy Constraints}

Sensor nodes are usually battery-operated and randomly deployed in inaccessible areas. Their batteries are not easily replaceable and they can be inconvenient to recharge. Since the nodes are continuously sensing, processing, and transmitting multimedia traffic, their energy can be easily depleted.

In addition to this, large traffic volumes also require extensive processing that is not suitable for the battery-operated network. Examples of processing methods used in WMSNs are data aggregation and compression. According to Znati et al [3], data aggregation refers to a set of operators such as MIN, MAX, AVERAGE, COUNT etc. which can be used to combine data into meaningful information. On the other hand, data compression in sensor networks is concerned with reducing the number of bits to be transmitted while maintaining the meaning of information. As an example, source coding techniques used for data compression in WMSNs are: MPEG-4 [23], DPCM/DCT [19], H.263 [17] and JPEG [24].

Even though energy can be obtained through thermal conversion, seismic and photovoltaic means, it may not always be the case in most environments [3]. Failure of the nodes to maintain their energy will cause the nodes to die, affecting the application and the network topology. However, energy can be saved by shutting down the nodes, switching into sleep mode or halting traffic generation.

\subsubsection{High Bandwidth Constraints}

WMSNs are usually deployed either on a small scale with homogeneous sensors or on larger scales consisting of heterogeneous sensor nodes. Multimedia sensor networks carry a high traffic volume because in addition to static data such as pressure and temperature, they carry video streams, still images and audio traffic. Generally, nodes that are located closer to the sink suffer the most, as they experience high traffic load because they carry combined traffic coming from other sensors. All these necessitate high data rates at the upstream links to meet the high bandwidth demand of the traffic. In WMSNs, video transmission rates may range from $100 \mathrm{kbps}$ for low quality video to $3 \mathrm{Mbps}$ for high quality video. When comparing multimedia demands for video, audio and images, the video will have the highest bandwidth requirement. This is because a $1 \mathrm{~GB}$ low quality video of one hour's duration needs at least a $2.5 \mathrm{Mbps}$ link for transmission. 
Meeting the multimedia data requirements is vital in ensuring that high quality video, images and audio are received at the sink. However, the high traffic volumes produced by multimedia data make it difficult to meet these requirements because they cause congestion, especially at the nodes located closer to the sink node. Controlling congestion in WMSNs is critical in preserving the visual quality of multimedia data, as well as prolonging the system lifetime. Congestion control is the main focus of this research.

\subsection{Congestion in WMSNs}

Congestion in WMSNs can be a complex issue because determining its cause and locating it in the network can be a difficult task. The manner and the conditions in which traffic is sent from the sensor nodes to the sink may also contribute to the complexity of congestion. This section describes the factors that contribute to congestion in WMSNs.

a) Delivery Models: In WMSNs, traffic can be delivered using one of the following models: continuous-based, query-driven, event-based or a hybrid model [25]. In the continuous-based model, traffic is transmitted at specified intervals to the base station. In query-driven mode, traffic is only transmitted upon a query by the base station. In the event-based delivery mode, traffic is transmitted upon occurrence of a certain event. The hybrid model is a combination of any of these three delivery models. In all these models, depending on the event, high multimedia traffic volume consisting of video streams and snapshots are sent in an upstream fashion, causing congestion, especially in sensor nodes which are closer to the sink. Nodes are normally deployed in large quantities spanning larger geographical areas making congestion even more likely to occur [26].

b) Link Level and Node Level Congestion: Congestion in WMSNs occurs either at the transmission media or at the individual node as indicated in Figure 2.2. At the transmission medium, congestion occurs as a result of nodes trying to access the link simultaneously; this is called link level congestion. Node level congestion occurs due to buffer overflow at the nodes. Buffers are temporary storage areas at the nodes where all the incoming and outgoing packets are stored. Data overflow happen because packets are transmitted at a faster rate than what they can be served at the buffers; therefore packets get backlogged 
and new packets can no longer be accepted at the nodes. This research focus on node level congestion.

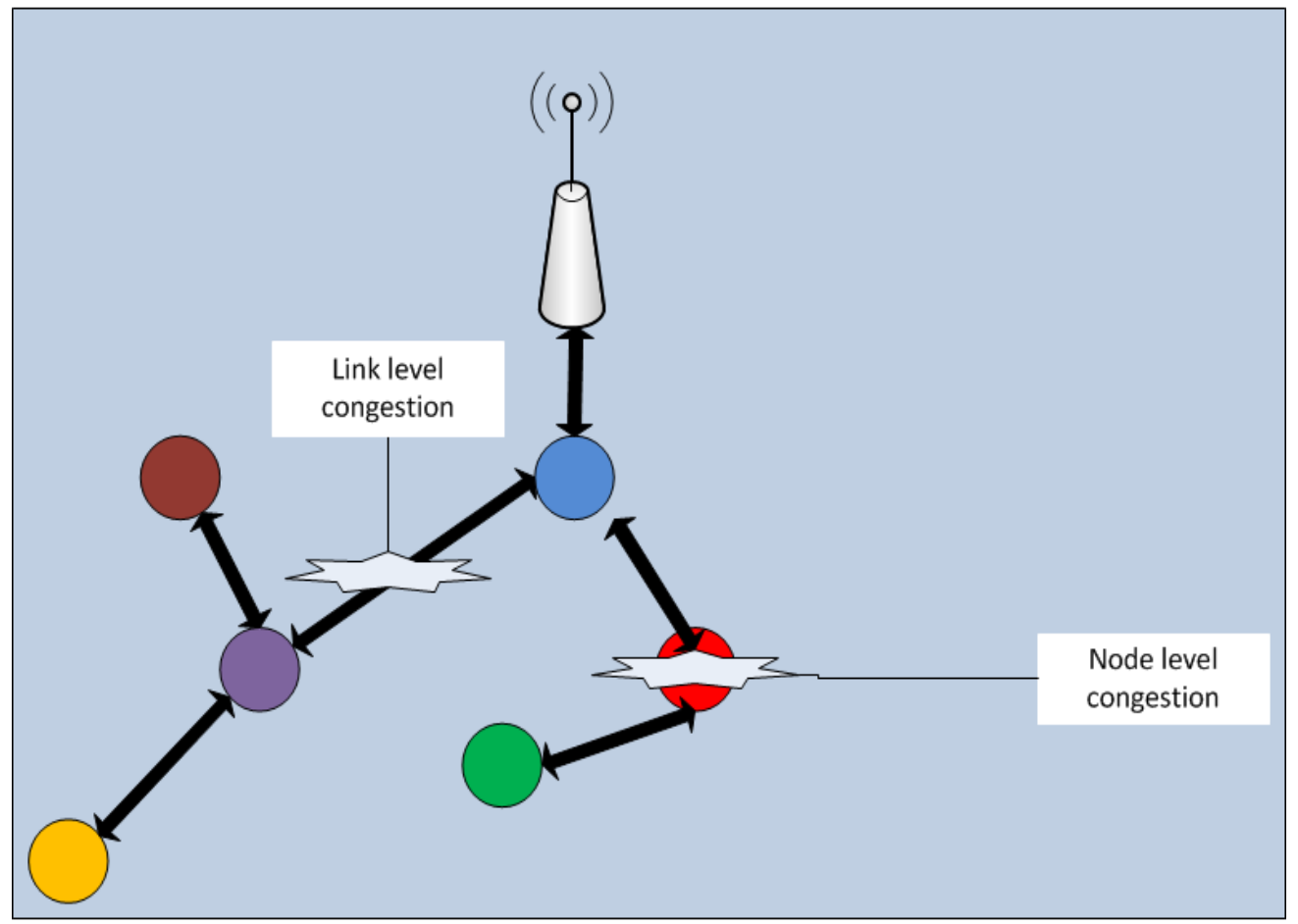

Figure 2.2: Link level and node level congestion

c) Other causes of congestion: Another factor that leads to congestion is the change in network topology. Nodes continue to die due to high energy consumptions causing the nodes that are left behind to take extra traffic loads, leading to congestion. Sensor nodes are also fitted with small storage and processing capacities which are insufficient for the high traffic volumes of multimedia data, resulting in further congestion.

As seen above, congestion in WMSNs is caused by several factors. Once congestion occurs, the multimedia application may experience packet delays, increased jitter and packet loss, while the network may suffer depletion of energy and decreased reliability [11]. In addition to this, link level congestion is also claimed to increase packet service time, and decrease both link utilization and throughput of packets [27]. These consequences may affect the traffic flow in the network and could cause the network to collapse. With this mind, it is very important to detect and control congestion in WMSNs so as to meet the QoS requirements for multimedia applications. 


\subsection{WMSNs Architectures}

One way to address congestion in WMSN is by re-structuring the architecture of the network to ensure fairness among resources. Over the past few years, a number of WMSNs architectures were proposed in the literature [28] [29] [30]. The architectures are expected to meet the resource-constrained nature of sensor nodes, but still accommodating the specific requirements of WMSNs.

Normally, a WMSN is composed of multimedia capable sensor nodes, wireless links, and a base station. Figure 2.3 depicts a general WMSN architecture. Each component's function is described below.

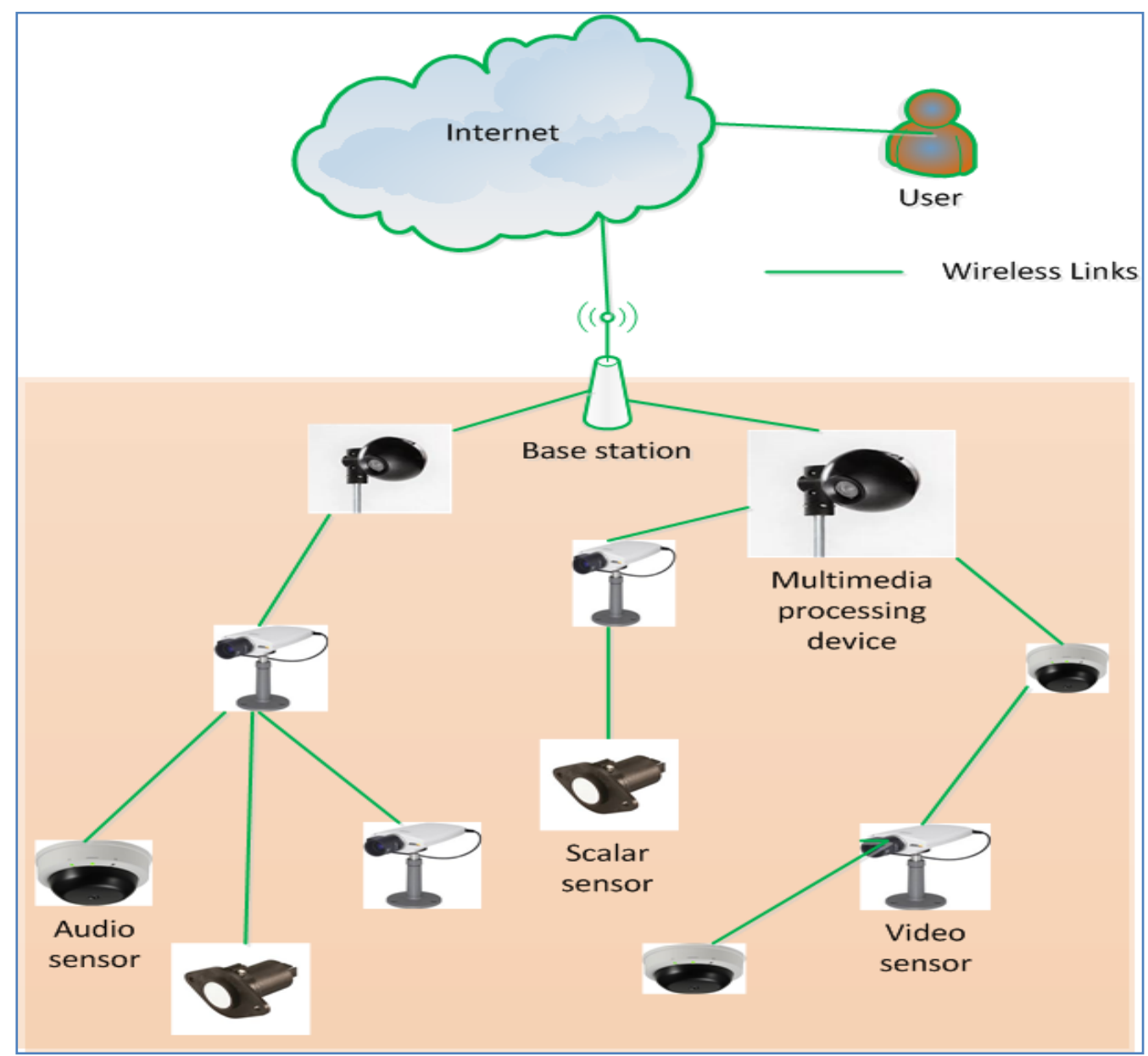

Figure 2.3: WMSN Architecture 


\subsubsection{WMSN Components}

a) Sensor nodes: These are the central components of the WMSN. Sensors are equipped with cameras and microphones to enable them to retrieve multimedia and scalar content from the environment. The nodes are each fitted with different resources as shown in Table 2.1. A sensor node can be either a child node or a parent node. Child nodes transmit their traffic to an attached node. The node that receives traffic from a child node is called a parent node. For example, in the diagram above, the scalar sensor is a child node and the video sensors to which they are attached are called parent nodes. Child nodes usually transmit their traffic through parent nodes, which may be fitted with more powerful resources than child nodes.

The nodes transmit two types of traffic, namely transit traffic and local traffic. Transit traffic is the traffic a node receives from directly connected nodes while local traffic is the traffic a sensor node generates by itself. The nodes can deal with different types of traffic with varying priorities depending on the type of information collected and the geographical position of the nodes.

Table 2.1 WMSN node features

\begin{tabular}{|l|r|r|r|r|l|}
\hline \multicolumn{1}{|c|}{ Feature } & Imote & Mica2 & MicaZ & Telos & Imote $^{\mathbf{2}}$ \\
\hline CPU type @[MHz] & $\begin{array}{r}32 \mathrm{bit} \text { ARM } \\
012\end{array}$ & 8 bit Atmel @8 & 8bit Atmel @8 & 16bit TI @8 & $\begin{array}{c}32 \mathrm{~b} \text { XS @ } 13 \\
(104)\end{array}$ \\
\hline SRAM [kB] & 64 & 4 & 4 & 10 & $256 / 32,000$ \\
\hline FLASH [kB] & 512 & $128+512$ & $128+512$ & $48 \mathrm{~KB} / 1024 \mathrm{~KB}$ & 32,000 \\
\hline Radio & $2.4 \mathrm{GHz}$ & $300-900 \mathrm{MHz}$ & $2.4 \mathrm{GHz}$ & $2.4 \mathrm{GHz}$ & $2.4 \mathrm{GHz}$ \\
\hline Data Rate [kb/s] & 720 & 15 & 250 & 250 & $250(720 / 11,000)$ \\
\hline Power sleep [mA] & $1-250$ & 19 & 27 & 6 & $1-100$ \\
\hline OS support & TinyOS & TinyOS & TinyOS & TinyOS & TinyOS \\
\hline
\end{tabular}


b) Base station/ Sink: A base station node or a sink node acts as an interface between the network controller and the sensor network. After a sensor node has collected data, it sends the data to a base station where it is further analysed by the network controller. A network controller may also query specific information related to the environment being monitored, such as the humidity or temperature at a certain point during a certain period. The sensor nodes will then perform data aggregation or compression to send the required data to the controller.

c) Radio transmission channel: This refers to the interface that transmits raw bit streams of the sensed data over the physical communication medium. The wireless technologies supported by WSNs are: Bluetooth (IEEE 802.15.1), Ultra-Wideband (IEEE 802.15.3), ZigBee (IEEE 802.15.4) and Wi-Fi (IEEE 802.11) [31]. However, IEEE 802.11, IEEE 802.15.1 and IEEE 802.15.4 are not suitable for WMSNs because they have high power consumptions [2] [32]. A promising wireless technology for WMSNs is the Ultra-Wide Band (UWB) that is able to support applications with high bandwidth requirements while using low power. The UWB uses the $3.1-10.6 \mathrm{GHz}$ frequency band and it can transmit up to $480 \mathrm{Mbps}$ within short distances [2]. The wireless channel plays a big role in the delivery of traffic to the sink, however, due to the physical constraints of wireless system such as the high bit error rates (BER) and signal-to-noise ratio (SNR), it is likely that most packets will not reach the sink. This requires powerful signal processing techniques to be applied to data before and after transmission.

Using the components and node features outlined above, a number of different architectures for WMSNs have been proposed in existing literature [2][30]. These architectures are scalable and are able to sustain the lifetime of WMSNs considering their limited constraints. The choice of which architecture to utilise is determined by the applications. The three architectures are: single-tier flat architecture, single-tier clustered architecture and multi-tier architecture.

\subsubsection{Single-tier Flat Architecture}

In a single-tier flat architecture, homogeneous sensors are equipped with equal sensing and processing capabilities [6][33]. In this architecture, every sensor node collects its own multimedia data, processes it and transmits it directly to the sink. A single-tier flat architecture offers benefits such as easy maintenance, low power demand and easy management. Since the 
nodes have equal capabilities, nodes are likely to deplete their resources at the same time. Figure 2.4 gives an example of a single-tier flat architecture.

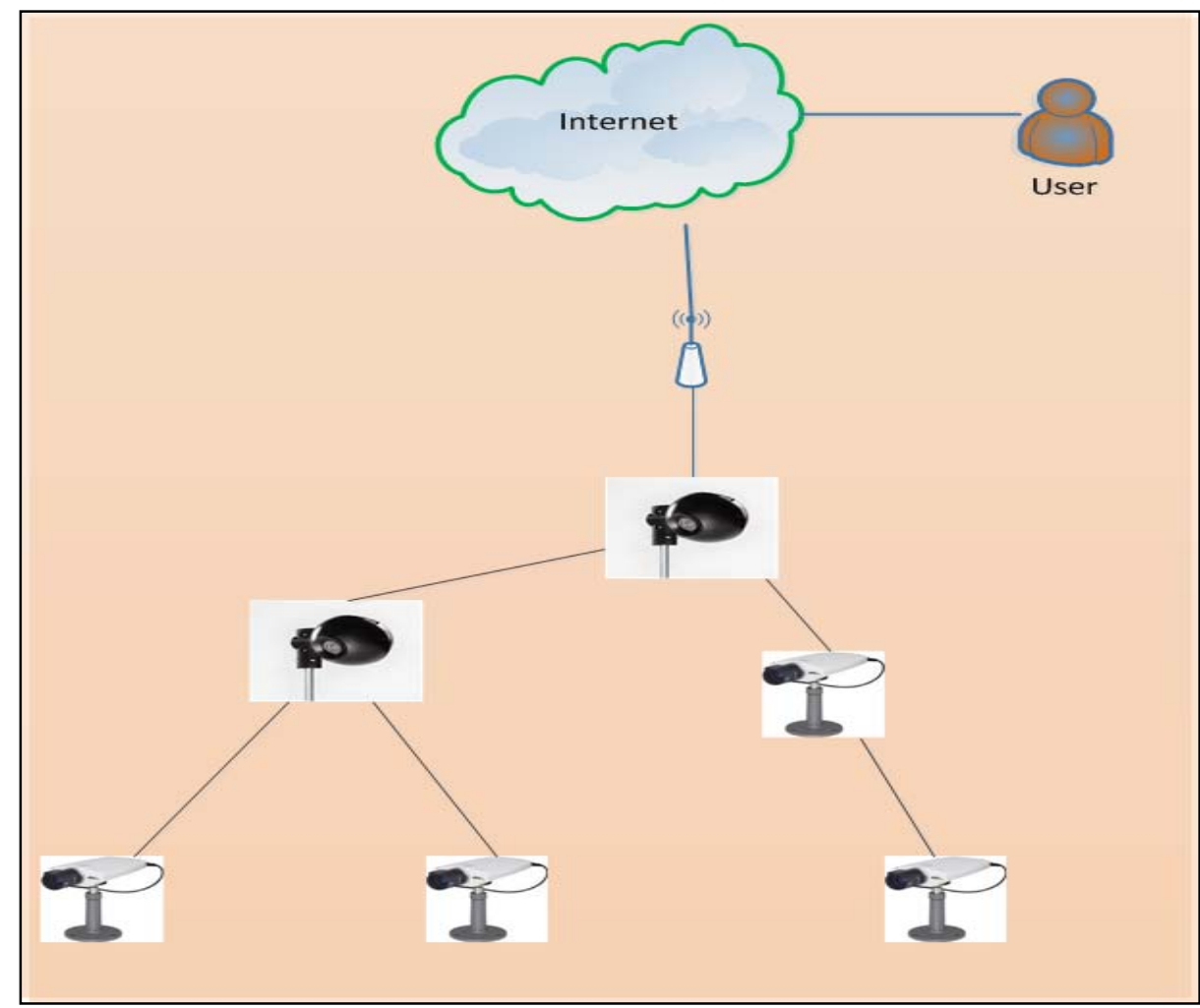

Figure 2.4: A single-tier flat architecture

\subsubsection{Single-tier Clustered Architecture}

In a single-tier clustered architecture, heterogeneous sensor nodes transmit multimedia traffic through a cluster head that has been selected by the sensor nodes or pre-assigned by a network engineer. The cluster head serves as the processing unit and is usually equipped with more computational and communication resources in order to perform complex operations on the information it receives. If every sensor node in the network is able to sense, process and transmit data, it would easily drain the energy of the network, thus the purpose of using cluster heads. The clustering of nodes offers benefits such as scalability, efficient bandwidth usage, long network lifetime and easy network topology maintenance [34]. Figure 2.5 depicts a singletier clustered architecture. 


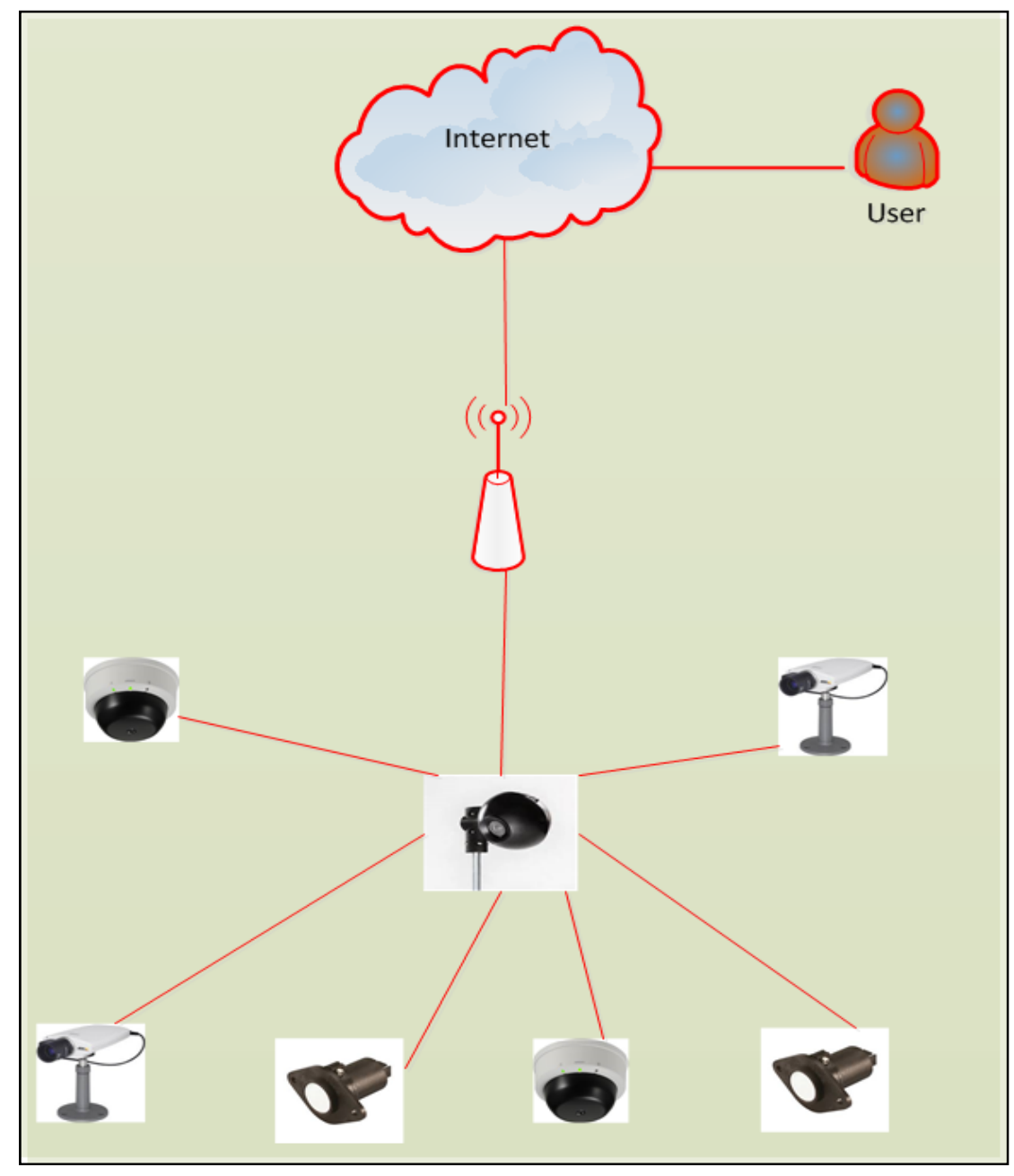

Figure 2.5: Single-tier clustered Architecture

\subsubsection{Multi-tier Architecture}

A multi-tier architecture consists of hierarchically multi-layered heterogeneous sensor nodes. The first tier generally consists of simple and generic sensor nodes while the middle tier consists of medium resource equipped nodes. The third layer, which is likely to be the last tier, is comprised of rich resources that are able to perform intensive functions. Typical examples of multi-tier architecture implementations are found in [28] and [35]. Multi-tiered architectures are easily deployed due to being reliable and less expensive, as well as the fact that they can cover larger areas. A multi-tiered architecture is shown in Figure 2.6. 


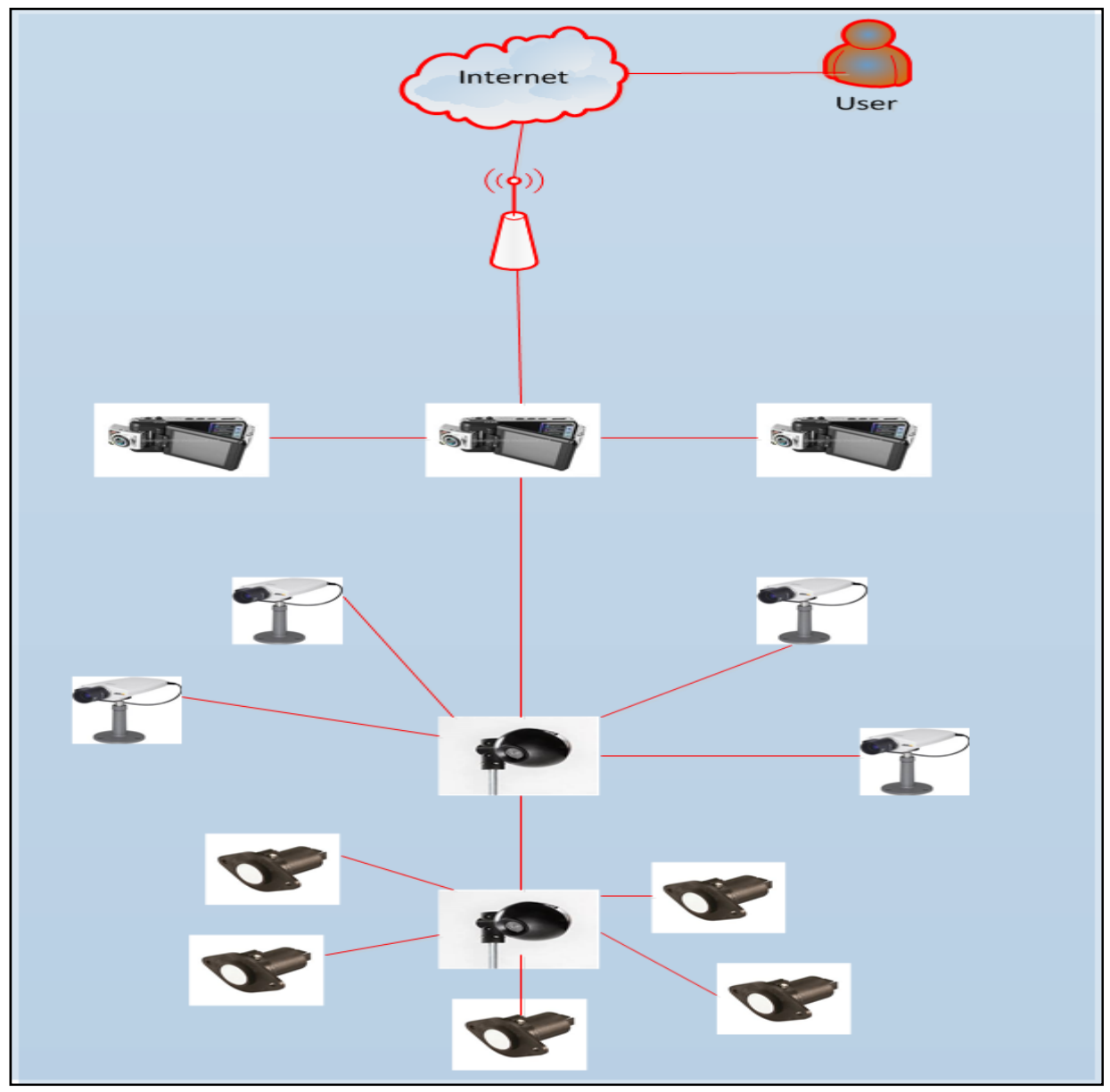

Figure 2.6: Multi-tier Architecture

In order to address the issues of congestion and to ensure that the QoS of the different applications are met in WMSNs, a reliable transport layer is required. The transport layer is designed to address the following services: reliability, fair bandwidth allocation, congestion control and energy efficiency [36]. The Transmission Control Protocol (TCP) which implements congestion control and reliability in the Internet cannot be used in WMSNs. This is because TCP demands acknowledgement during packet retransmission that is not desirable in WMSNs because it will contribute towards the depletion of energy at the nodes [37]. Another transport layer protocol used in traditional internet communication is User Datagram Protocol (UDP). However, UDP is also not suitable for WMSNs because it is not reliable as it does not guarantee delivery of packets. Efficient congestion control protocols for WMSNs that guarantee energy efficiency, QoS support and fairness among transmitted traffic [38], therefore need to be implemented. 


\subsection{Congestion control protocols in WMSNs}

In recent years, several congestion control protocols have been proposed for WMSNs [39][40][41]. These protocols can be divided into four categories depending on how they detect the presence of congestion and how they control congestion. The categories are: I) Queue assisted protocols, (II) Priority aware protocols, (III) Resource control protocols and (IV) Content aware protocols.

Most of the existing congestion control protocols consist of three units, namely congestion detection unit, congestion notification unit and rate adaptation unit. The congestion detection unit is responsible for detecting the presence of congestion in the network. Several methods of detecting congestion proposed so far include packet service time and inter-arrival time [40] [38], queue length [42][43], channel load [16] or a combination of these parameters [14]. The congestion notification unit is concerned with disseminating congestion-related information once congestion has been detected. Two types of congestion notification techniques exist: namely implicit and explicit notification. Implicit notification piggybacks congestion information in a packet header while explicit notification makes use of a special extra packet to carry congestion information to other connected nodes.

Common methods used to control congestion are traffic control and resource control. Congestion control can further be classified as end-to-end or hop-by-hop. In end-to-end control, the end nodes are responsible for performing rate adjustments, while in hop-by-hop, intermediate nodes perform rate adjustments. Other methods used to handle congestion are through routing optimization and data processing [37]. Some of the existing congestion control protocols used in WMSNs and WSNs are discussed below.

\subsubsection{Queue Assisted Protocols}

Queue based protocols are concerned with keeping queue levels low at the sensor nodes. Examples of queue based protocols are [42][16][43].

Congestion detection and avoidance (CODA) [16] is an energy efficient congestion control protocol aimed at controlling both link level and node level congestion in WSNs. CODA consist of three levels, namely congestion detection, open loop hop-by-hop backpressure and closed loop multisource regulation. The transmission medium and the queue level are used to detect the presence of congestion in the network. This is achieved by periodically sampling the channel condition and then comparing the fraction of time that the 
medium is occupied to the optimal channel utilization. Once congestion is detected, broadcast backpressure messages are sent towards the source nodes in order for them to adjust their rates or to drop packets depending on the congestion policy. Upon receiving the backpressure message, a node can decide whether to send this message further to its neighbour nodes. The source node can regulate its own rate or the sink can compute the rate for the source. As long as the source rate is less than a fraction of the maximum theoretical throughput, the source controls its own rate. When this maximum theoretical throughput is exceeded, the sink will regulate the source traffic.

In order to evaluate the performance of CODA, NS-2 and real sensors were used. The number of sensor nodes used ranged between 30 and 120 and were randomly placed. The results show that CODA is able to save nodes' energy, and has low packet loss and high delivery of packets at the sink [16].

Despite achieving good results during its performance, the Additive Increase Multiplicative Decrease (AIMD) traffic regulation technique used in CODA poses several drawbacks. Firstly, AIMD often leads to packet loss, queue length jitter and low link utilization, which is undesirable for real-time traffic. Secondly, during network congestion, the closed loop multisource regulation used may lead to high delays and high error rates. These too have negative effects on the real-time data. Thirdly, when congestion occurs, the backpressure messages that are used may easily create overhead which may decrease the energy and bandwidth of the sensor nodes. Another disadvantage of CODA is that it does not support differentiated services, meaning all the traffic is treated the same regardless of its level of importance. However, WMSNs deals with different traffic types, therefore it is important to support differentiated services to ensure that the service requirements of different traffic are met.

Ee and Bajcsy introduced Fusion in [42]. Fusion uses three techniques that operate at different layers of the network protocol stack to control congestion in wireless sensor networks. These techniques are hop-by-hop flow control, prioritized Medium Access Control (MAC) and rate limiting. Hop-by-hop flow control deals with detecting the presence of congestion by using buffer occupancy and channel sampling. During congestion, a congestion bit is set in the header of every packet being sent from the congested node. Due to the broadcast nature of wireless networks, other nodes in the network will overhear the congestion bit and will not transmit to it. This will stop other nodes who wish to transmit to congestion nodes from doing so as their packets will be dropped due to buffer overflow. Rate limiting is concerned with lowering the 
transmission rates of sensor nodes while the prioritized MAC uses back-off techniques in order to allow congested nodes to transmit their data.

Fusion was tested using a wireless sensor testbed with 55 nodes. The results show that Fusion can achieve fairness, channel loss rates and good throughput. Despite the fact that Fusion achieves good throughput and fairness, its non-smooth rate adjustment harms link utilization and fairness [44]. Like CODA, Fusion does not support differentiated service and it also does not take the priorities of traffic into account during its rate adjustment.

\subsubsection{Priority Aware Protocols}

Priority based protocols assigns priorities to sensor nodes to ensure that certain nodes receive priority-dependent throughput. It is in this category that our proposed protocol is found.

PCCP [38] is an upstream congestion control protocol which addresses both link level and node level congestion in single-path and multi-path networks. Like other congestion control techniques, PCCP consist of three units, namely intelligent congestion detection, implicit congestion notification and priority-based rate adjustment.

PCCP uses packet inter-arrival time and packet service time to infer congestion. The values calculated by these parameters can indicate whether there is congestion in the network. Congestion notification is piggybacked in the header of data packets in order to inform other nodes about the congestion levels in the network. Once there is congestion in the network, every sensor node is able to perform rate adjustment to assure that 1) nodes considered to be of high importance receive more bandwidth, 2) nodes having the same priorities get equal bandwidth and 3) nodes that have more packets to send get sufficient bandwidth.

PCCP has several known weaknesses as outlined in [41]. The first known weakness of PCCP is the unfairness of the rate adjustment during the different congestion conditions. In PCCP, when there is no congestion in the network, both the scheduling rate and rate at which traffic is generated are increased for all the nodes without considering the priorities of the nodes, while when there is congestion in the network, the transmission rates are decreased depending on the priorities of the nodes. The priorities of nodes and traffic need to be taken into consideration every time the rates are changed to ensure that high priority data obtains more throughput regardless of the state of the network. Secondly, PCCP does not consider service differentiation. This means that all traffic types dealt with in WSN, real-time and nonreal-time multimedia are treated similarly when being processed at all the nodes in the network. 
This however is inappropriate as the different traffic types have several and specific QoS requirements such as delay, packet loss and throughput. The last weakness of PCCP is that it considers constant service time of packets which cannot be relied on as the wireless transmission media is prone to changes [41].

Another priority-based congestion control protocol was proposed in [45]. In this protocol, traffic is divided into four (4) traffic classes: real-time traffic (EF class), high priority non real-time traffic (NRT1 class), medium priority non real-time traffic (NRT2 class) and low priority non real-time traffic (NRT3 class). This is done to support service differentiation among the different traffic types in WMSNs. PBRC-SD employs a technique similar to random early detection (RED) [46] to detect congestion.

In the RED active queue management algorithm, two threshold values, $\operatorname{Min}_{t h}$ and $\operatorname{Max}_{t h}$, are defined. The average queue length at the node is compared against the threshold values to determine the congestion level. The output of the congestion level will cause the rate adjustment technique to increase or decrease the nodes' transmission rates bearing the nodes' priorities in mind. Implicit congestion notification is used to notify the rest of the nodes about their new rates after the rate adjustment has been performed. In order to test the performance of PBRC-SD's, simulations were carried out and the PBRC-SD protocol was compared against $\mathrm{CCF}$ and PCCP. Simulation results indicate that PBRC-SD achieved high throughput, low packet loss probability and low queueing delay when compared against the other two protocols.

PBRC-SD has several known weaknesses. Firstly, results from our analysis show that the threshold values in RED need to be constantly adjusted in order to obtain an accurate indication of congestion in the network. Secondly, another analysis of RED [47] shows that when the average queue size is above $\operatorname{Max}_{t h}$, the amount of packets that are successfully received at the sink is highly affected. The last drawback of PBRC-SD is that it only supports single-path routing in which traffic only have one dedicated upstream hop. However, WMSNs produces bursty traffic that can easily deplete the energy of either the upstream nodes or the nodes generating traffic. Thus, the use of single-path routing alone is not suitable for routing WMSN traffic.

$\mathrm{CCF}[40]$ is a scalable algorithm which uses packet service time to detect the presence of congestion in the network. Every node measures the average rate at which packets can be sent from the nodes, divides it among the child nodes and then updates the new rate. It uses implicit notification to notify other nodes of the presence of congestion. Exact rate adjustments 
are performed depending on the service rate and available child nodes. This protocol is implemented in the transport layer and it can work with all types of MAC protocols. CCF mostly focuses on achieving fairness among the sensor nodes; however, this may result in low throughput especially when certain nodes are not transmitting packets.

A known weakness of CCF is low utilization of the available capacity when certain nodes do not have any data to send or when they become idle for a long period [41]. Its rate adjustment strategy also yields low utilization because it depends on the service time to calculate the new rates. As shown in [39], CCF is a non-work-conserving algorithm whereby a node can only receive traffic from another node once the required number of packets has been received. Another problem of CCF is that the traffic generated is treated the same, meaning traffic with specific service requirements is not guaranteed to receive the necessary throughput, packet loss or queueing delay it requires.

\subsubsection{Resource Control Protocols}

Resource control protocol mitigates congestion by creating alternative paths to the base stations during congestion.

One such protocol was proposed in [15]. Once there is congestion detected in the network, HTAP utilises unused nodes to transmit packets to the sink. It achieves this by controlling the topology, hierarchical tree creation, alternative path creation and handling of powerless nodes. In the topology control scheme, a Local Minimum Spanning Tree algorithm (LMST) is used for preserving the network connectivity. Path creation and flow establishment are carried out in the hierarchical tree creation, while an alternative path runs before congestion can take place. The powerless nodes scheme is concerned with getting rid of nodes that have failed.

Another resource congestion control protocol was proposed in [13]. This protocol considers the content of multimedia whereby packets consisting of I-frames are kept during congestion. This is because the I-frames are considered to be the most important frames in multimedia content and the loss of such packets has a large impact on the quality of the video. Two types of protocol known as Source Congestion Avoidance Protocol (SCAP) and Receiver Congestion Control Protocol (RCCP) are used to predict the level of congestion in the network. The two protocols further adjust the transmission rates of the source nodes and the forwarding nodes. 
It has to be noted that congestion in WMSN is not permanent, but only happens for a short while and therefore availing more resources to the network can be a waste as most of the resources will not be utilised throughout the lifespan of the sensor network. Due to this reason, resource control protocols are not appropriate for reducing congestion in WMSNs [27].

Motivated by the limitations of the existing protocols such as PCCP [39], CCF [40] and PBRC-SD [45], a priority-based rate routing protocol for WMSNs will be proposed in this research. The proposed protocol is aimed at improving PBRC-SD and has thus borrowed some features from it.

\subsection{Chapter Summary}

In this chapter, a discussion of congestion and its effects on WMSNs has been presented. The challenges and requirements of WMSNs have also been discussed, as well as the architectures that are supported in the implementation of WMSNs. This chapter has been concluded by a discussion of existing congestion control protocols applicable in WSNs and WMSNs. 


\section{CHAPTER 3}

\section{SYSTEM DESIGN AND METHODOLOGY}

In the previous chapter, existing congestion control protocols for WMSNs were discussed. Different priority-based congestion control strategies are proposed in the literature. These are CCF [40], PCCP [39] and PBRC-SD [40], which try to alleviate congestion by adjusting the transmission rates of nodes. In PCCP and PBRC-SD protocols, the rate adjustment algorithms consider the nodes' priorities during the allocation of transmission rates. However, PCCP and PBRC-SD have several limitations. For example, the PBRC-SD protocol is energy inefficient and has difficulties anticipating QoS parameters. PCCP's weakness lies in its rate adjustment algorithm, which does not always consider the priorities of the nodes when adjusting the transmission rates. These limitations clearly motivate the need for better prioritybased congestion control protocols for WMSNs, which address the QoS requirements of realtime multimedia traffic while meeting the energy requirements of WMSNs.

\subsection{Introduction}

In this chapter, an energy efficient priority-rate based congestion control protocol called Priority-Rate Based Routing Protocol (PRRP) is proposed. PRRP improves the existing PBRCSD protocol that uses RED to detect the presence of congestion in a single-path network. However, unlike the PBRC-SD protocol, PRRP uses an adaptive RED queue that automatically adjusts the queue parameters to meet the change in the wireless sensor network conditions and the energy of nodes as criteria to decide on the best path to route data in a multi-path wireless sensor network.

Since the WMSNs consist of different applications producing various traffic types, this traffic may have varying service requirements. Therefore, PRRP also supports service differentiation to ensure that the multimedia traffic requirements are met. The proposed routing protocol operates at the transport layer of the network stack.

\subsection{PRRP Design}

As mentioned in Chapter 2 Section 2.2, WMSNs consist of sensor nodes that generate and transmit multimedia data to a base station. These data require low delay, low jitter, low packet loss and high throughput as it propagates through the network. It is very important to 
consider these strict service requirements especially when designing architectures and protocols of WMSNs to ensure that the quality of the data transmitted is not compromised. The purpose of this section is to describe how the sensor nodes used in PRRP are designed to meet the service requirements of multimedia data.

\subsubsection{Sensor Node Model}

\subsubsection{Service Differentiation}

In multimedia sensor communication, a combination of audio nodes, video nodes, image nodes and scalar nodes are wirelessly connected to measure scalar data and multimedia data from the environment. These nodes can either act as source nodes, relay nodes, source with relay nodes or sink nodes. Source nodes generate multimedia streams and scalar data and send it to the relay nodes which forward the data to the sink node. Source and relay nodes are capable of generating and forwarding data at the same time.

All the data generated and forwarded by the relay nodes is sent to the sink node which stores these data or records them to a separate storage device for further analysis. The data are generally analysed at a central site or a control office which is connected to the sink through an Internet's gateway. To ensure that the nodes do not deplete their resources at the same time, they are equipped with varying resources depending on the tasks they are performing. Source nodes are usually fitted with limited resources while relay and sink nodes are equipped with rich resources as they perform extensive processing of the data.

In PRRP, we assume that the multimedia traffic communicated by the relay nodes have strict service requirements, which can be determined by the source nodes' geographic location or by the type of traffic obtained by the source nodes. For example, traffic sent by a real-time streaming application has strict packet loss, packet delay and bandwidth limitation requirements, while traffic generated by thermal sensors can tolerate packet loss and packet delay. The sensor nodes that generate traffic with similar characteristics to that of the real-time streaming application are considered to be more important than that generated by the thermal nodes, thus the multimedia sensor network must ensure that it provides more resources to the sensors which are carrying important traffic.

In order to ensure that more resources are availed to the important traffic, the traffic generated by the source nodes is grouped into four different classes to accommodate the various WMSN applications. The traffic classes are: High Priority Real-Time traffic (HP-RT), High 
Priority Non real-time traffic (HP-NRT), Medium Priority Non real-time traffic (MP-NRT) and Low Priority Non real-time traffic (LP-NRT). The traffic classes are priority dependent and their order in terms of priorities are: HP-RT, HP-NRT, MP-NRT and LP-NRT. The different traffic classes are discussed below.

1. HP-RT: This traffic class consist of event driven multimedia streams that need to reach the sink in real-time. Such traffic is time critical and it is expected to reach the sink as soon as possible. A typical example of such traffic in WMSNs is the live video streams of an alarm signalling gas leakage in an oil rig. This multimedia traffic requires strict packet delay, low jitter, high throughput, high reliability and low packet loss. The traffic belonging to the HP-RT class has high bandwidth requirements and is assigned more resources by the network when compared to the other traffic classes. This is done to guarantee that its strict service requirements are met.

2. HP-NRT: Traffic in this class refers to important video and audio streams that do not need to reach the sink in real-time. This data is often processed off-line and is characterised by low delay and low packet loss. Scalar data of patterns observed by sensors in environmental monitoring is one example of such traffic. HP-NRT is not urgent and can be sent to the sink when several nodes have little or no traffic to send.

3. MP-NRT: Medium priority non real-time traffic is not mission critical and can reach the base station with packet loss and packet delay. Traffic that belongs to this class does not require high bandwidth. The data sent during a specified interval reporting on the current state of the electrical equipment in a certain industry is an example of traffic that belongs to this class.

4. LP-NRT: Data in this traffic class is not important and failure to reach the base station will not have a negative effect on the network. Therefore, this traffic tolerates packet loss and packet delay. Examples of LP-NRT data are the messages generated upon inquiry of the current state of the roads in road traffic management. 


\subsubsection{Queuing Model}

The four traffic classes discussed in the previous section are considered to ensure that the QoS service requirements of traffic with various priorities are met. Each of these traffic classes are further assigned their own queues.

The queueing model at each node is depicted in Figure 3.1 below and is described as follows:

1. When a packet arrives at each sensor node, it will queue in the incoming traffic buffer.

2. The speed of the node, the link speed and the traffic load will determine the extent of queueing at the buffer. When queueing occurs at the incoming traffic link, it may result in packet loss and packet delays.

3. Depending on the status of the queue, the packet leaves the incoming traffic buffer and proceeds to a classifier that checks the differentiated service field in the packet header and assigns it to one of the queues HP-RT, HP-NRT, MPNRT, or LP-NRT. Packets are only allowed to join one queue at a time.

4. After the packets have been placed in the appropriate queue, a weighted round robin (WRR) scheduler is used to serve the queues.

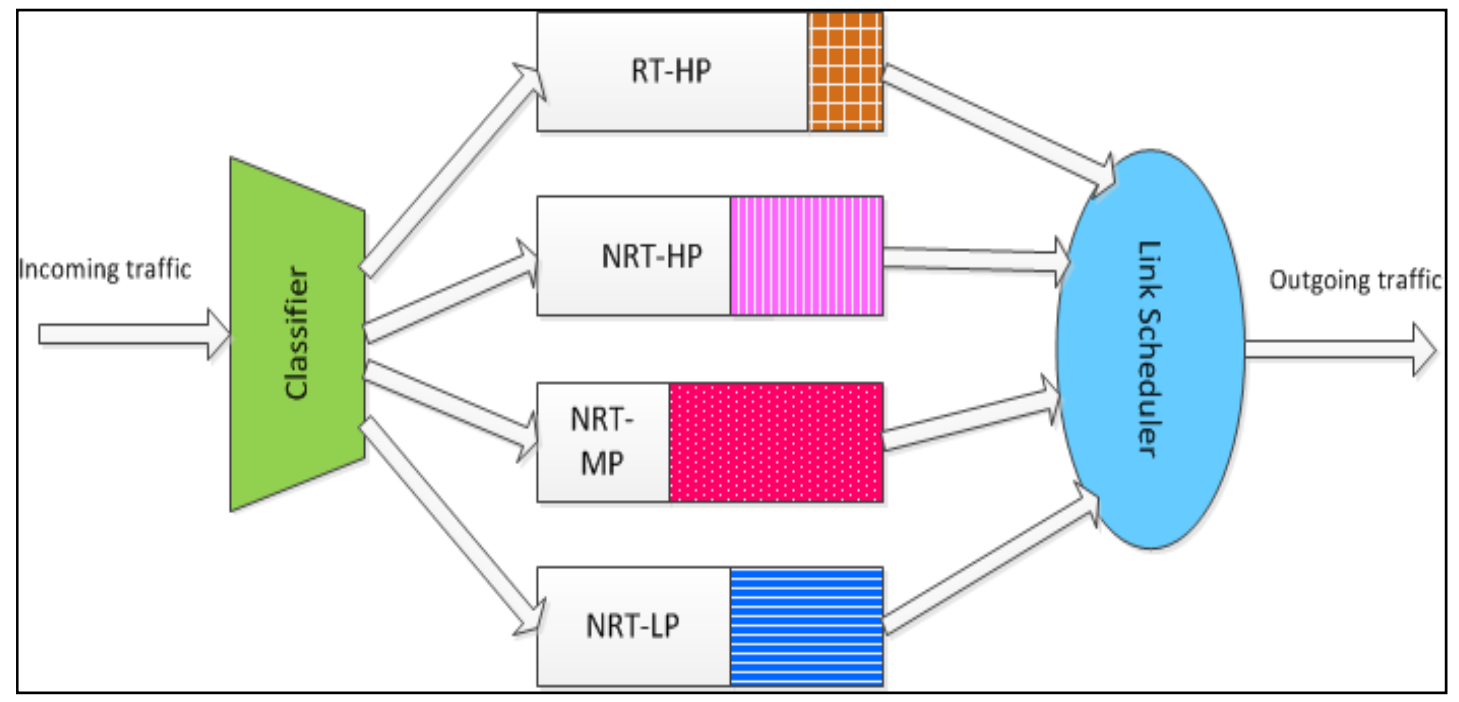

Figure 3.1: PRRP queueing model

\subsubsection{WRR Scheduler}

In WRR, each traffic queue $i$ is assigned a weight, $w_{i}$, which determines the amount of service it receives for each round. These weights are assigned depending on the priority of 
traffic such that HP-RT traffic obtains the highest weight, followed by HP-NRT traffic, followed by MP-NRT traffic and lastly LP-NRT. The operation of WRR is illustrated in Figure 3.2. As it can be seen in the diagram, the scheduler operates in a round robin manner, where it begins by scheduling the HP-RT class, followed by HP-NRT class, followed by MP-NRT class, and lastly LP-NRT class.

Assuming that each node $k$ has $n$ classes, each class will receive a fraction of service given by:

$$
S_{(i)}^{k}=\frac{w_{i}}{\sum_{j=1}^{n} w_{j}}
$$

where $S_{(i)}^{k}$ is the service share of queue $i$ at node $k$.

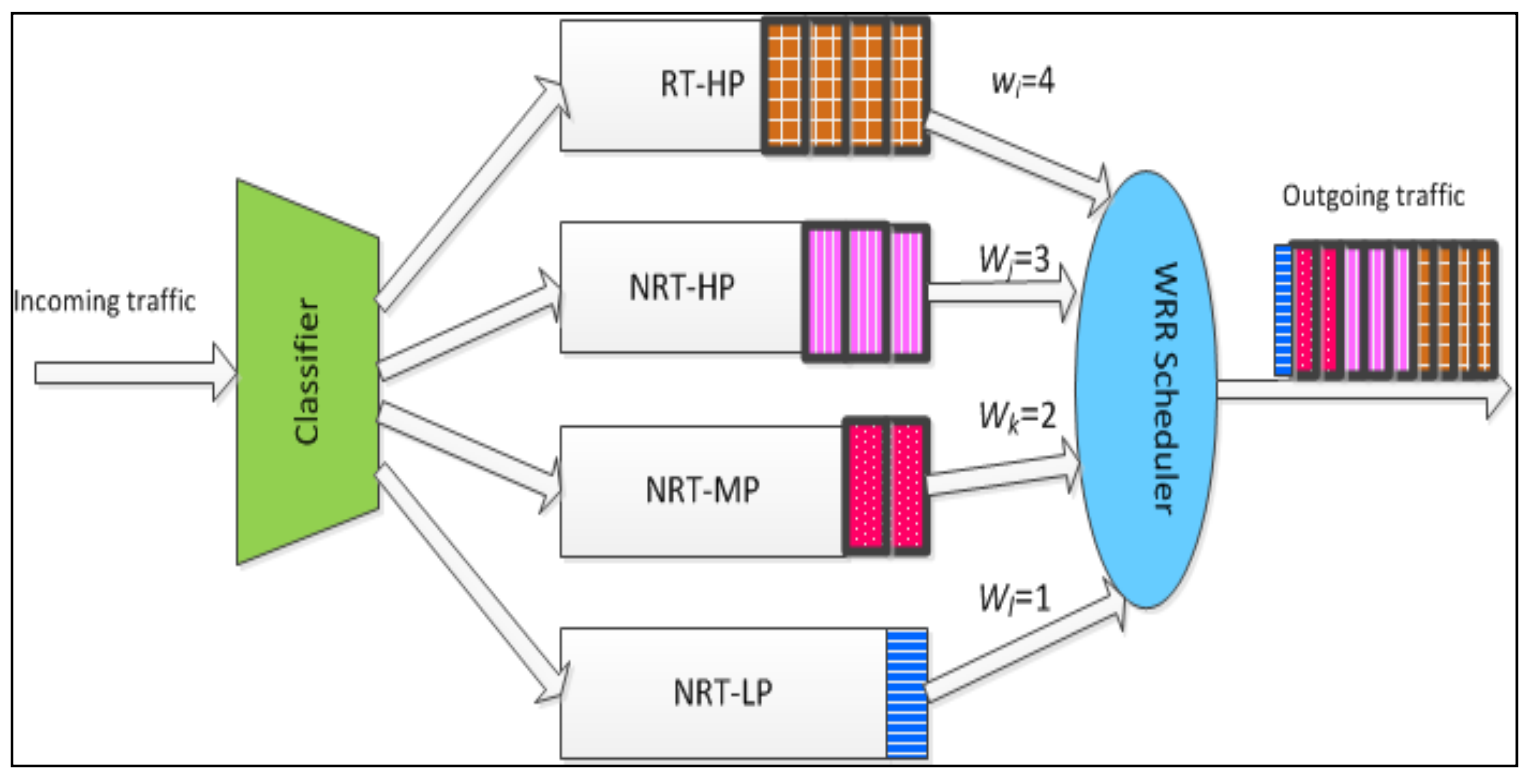

Figure 3.2: WRR Scheduler

WRR is suitable for scheduling multimedia traffic because it provides fairness, since the servicing of queues does not depend on the other queues' traffic flows. Another reason why WRR is preferred to a First In First Out (FIFO) scheduler for example, is that it is designed to better handle traffic classes with different requirements, which is the case for our system design [41]. After packets have been served by the scheduler, they go to the outgoing link where they are sent on to the appropriate node. 


\subsubsection{Network Model}

Generally, large numbers of sensor nodes are connected together through radio links to create a wireless multimedia sensor network. Each sensor node collects physical data and shares it with other nodes to enable the network to achieve its application goals. Sensor networks are deployed in various settings, such as homes, hospitals, the military and in industries, with the aim of monitoring a physical phenomenon and reporting it to a controller.

Two different types of architectures are considered in this research; a single-tier with single-path routing architecture and a single-tier with multi-path routing capabilities as shown in Figures 3.3 and 3.4 respectively. Both architectures are classified as single-tier clustered architecture as discussed in Chapter 2, Section 2.4.2. In PRRP, it is assumed that each sensor node can serve as either a parent node or a child node that is able to generate multimedia streams and scalar data belonging to either traffic class as discussed in the previous section.

Whereas in [41], the authors only considered a single-path network, in this research we have extended their network model to support multi-path routing where more than one path exists between child nodes and their parent nodes.

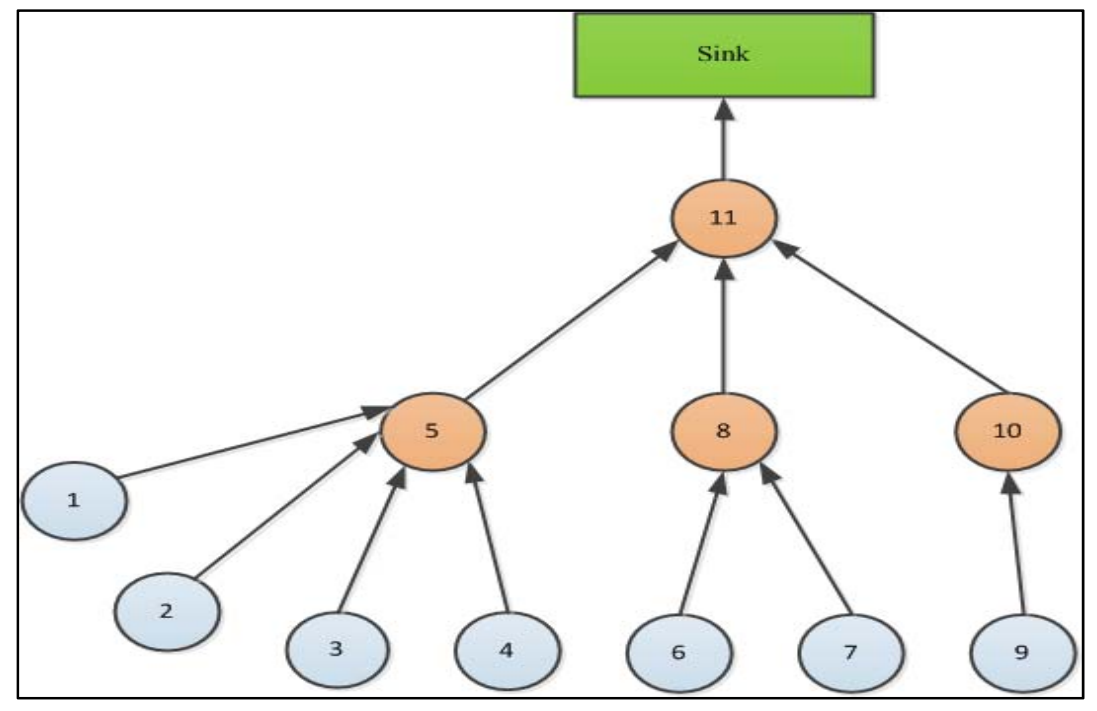

\section{Figure 3.3: Single-path Routing with extra parent node as considered in PRRP}

Multi-path routing offers benefits such as load balancing, high reliability and high resilience which can increase the network lifetime by spreading energy utilization among the sensor nodes. 


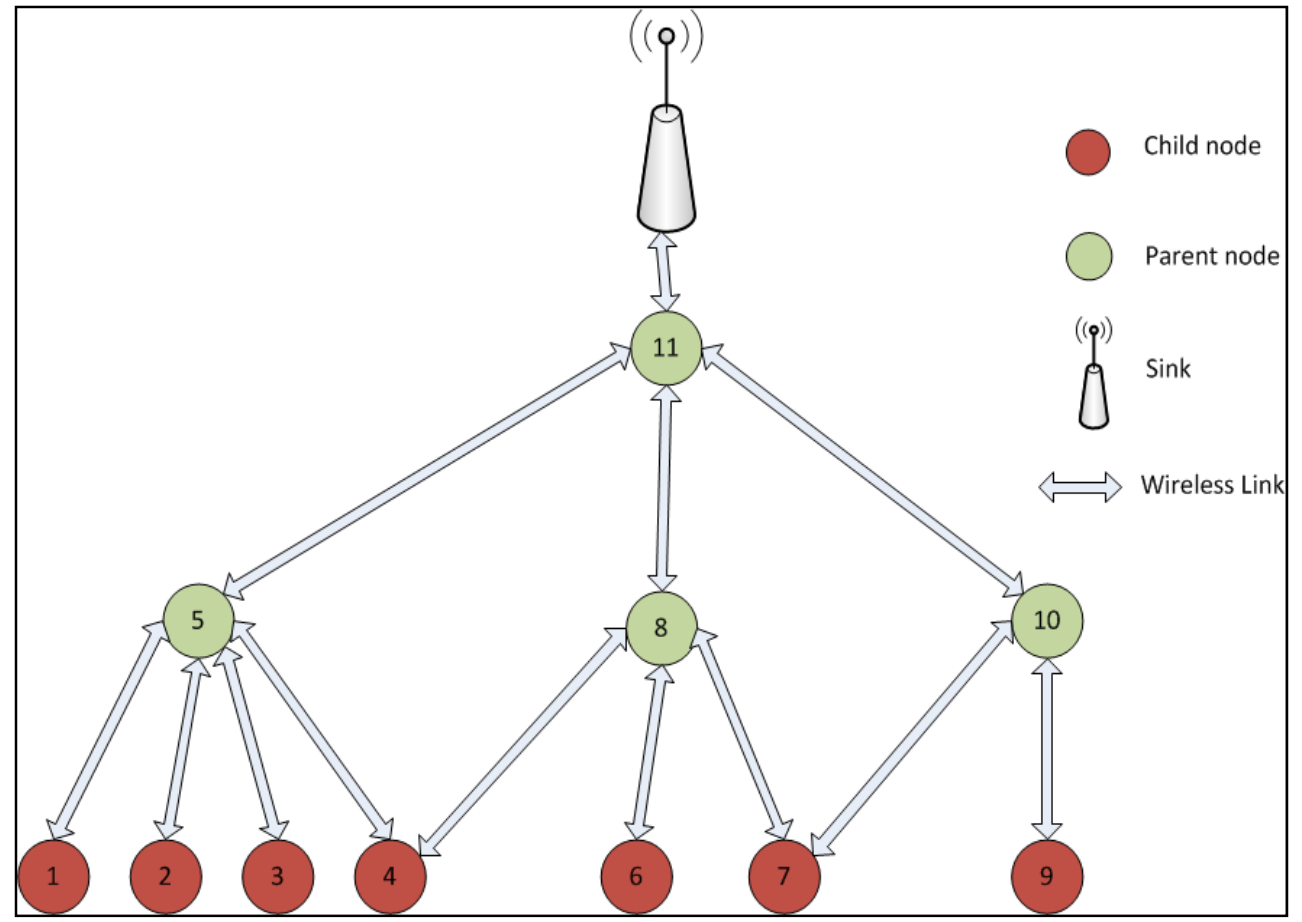

Figure 3.4: Multi-path Routing Network

For example, in our network above, node 4 and node 7 have two paths to choose from when routing traffic. Node 4 can either send traffic to node 5 or node 8 , while node 7 can either send through node 8 or node 10 . Any node in the network can be configured to perform multipath routing; however, enabling all nodes to perform multi-path can easily deplete the nodes' energy because of the duplicate data that is being forwarded by the nodes. Thus, in this research, only two nodes are enabled to perform multi-path routing. The critical issue in a multi-path routing network is how a child node should select the parent node that will relay its data. In this research, we propose one way of doing this, described in Section 3.3.5 below. 


\subsection{The PRRP Proposed Model}

PRRP is an energy efficient priority-based rate congestion control protocol aimed at reducing node level congestion by adjusting and assigning priority-based rates to sensor nodes. In node level congestion, packets experience delay and losses due to buffer overflow. At the node level, nodes have a better view of the various queues' behaviour and the average queue size which is used in congestion detection. The PRRP protocol operates smoothly by allocating higher rates to real-time traffic depending on the congestion status of the nodes. This section explains how congestion control will be achieved in PRRP.

\subsubsection{Congestion control levels of PRRP}

Similar to the existing congestion control protocols, our proposed protocol has three fundamental stages of controlling congestion. The stages are depicted in Figure 3.5 below:

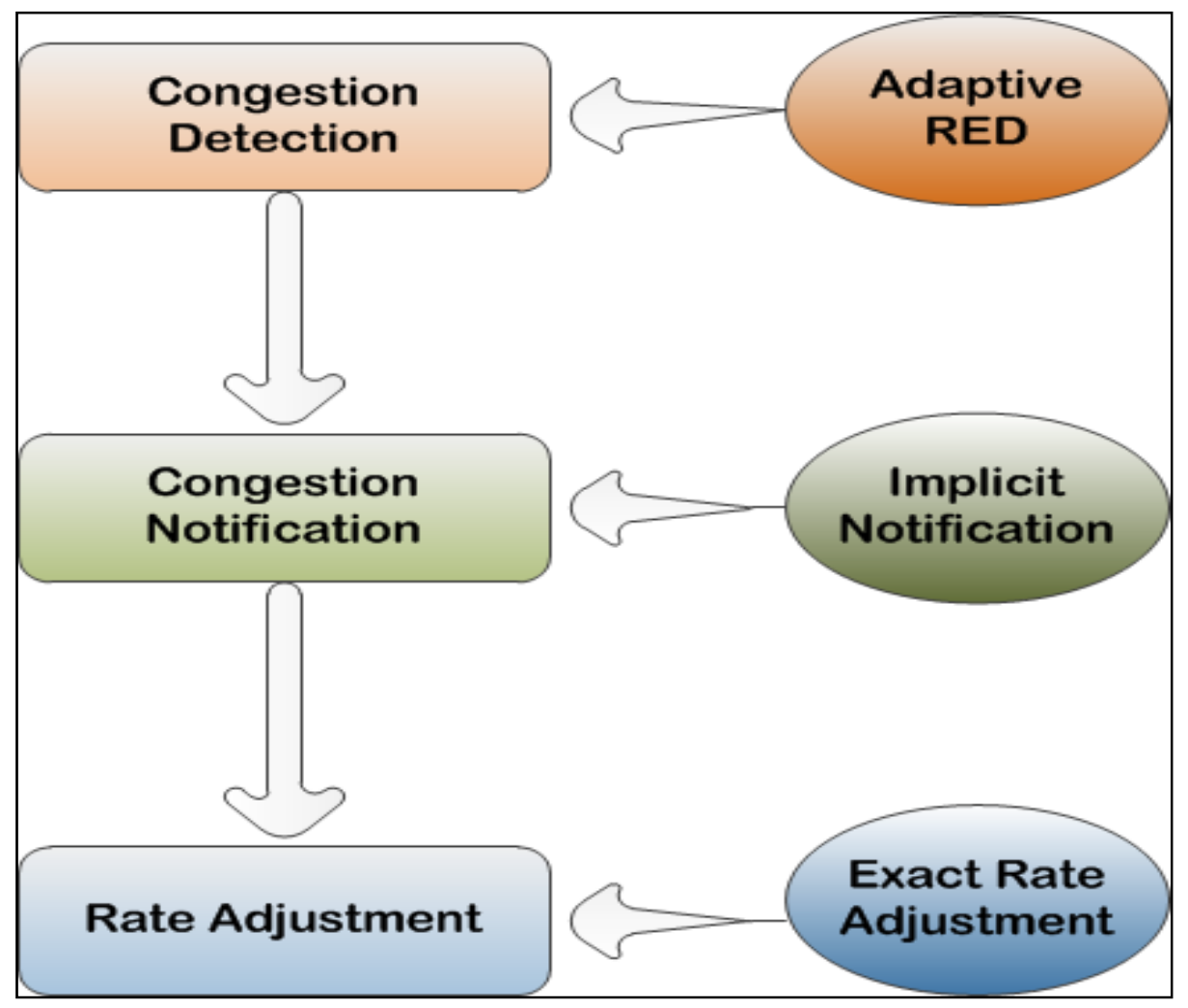

Figure 3.5: Congestion Control Stages 
The congestion detection unit is the first level of congestion control and it is responsible for detecting the presence of congestion in the network. A technique similar to RED is used to detect congestion in PRRP. Upon detecting the presence of congestion, implicit congestion notification will notify the rest of the nodes of the presence of congestion, resulting in the nodes adjusting their transmission rates. During rate adjustment, the priorities of the traffic and nodes are taken into account.

The overall flow of operation for the proposed protocol is shown in Figure 3.6 below.

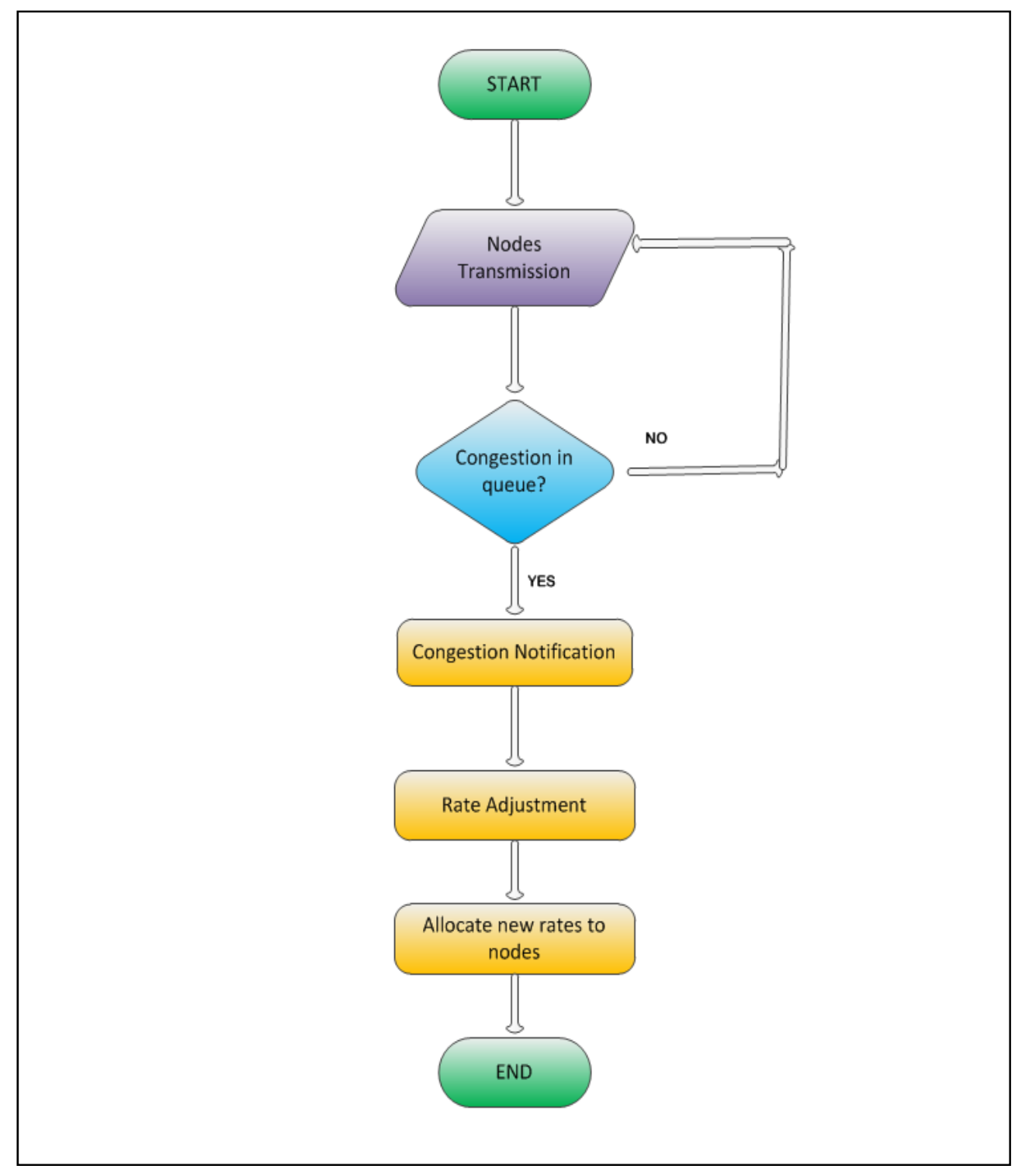

Figure 3.6: Step by step operation of PRRP 


\subsubsection{Congestion Detection in PRRP}

\subsubsection{Adaptive Random Early Detection}

Our proposed protocol utilises a technique similar to Random Early Detection (RED) for detecting congestion in WMSNs. In RED, congestion is detected by monitoring the queue length at the sensor nodes. The queuing model at each node is depicted in Section 3.2.1.2. Congestion detection in RED is achieved by setting two $k^{\text {th }}$ queue's threshold values, the minimum threshold $\operatorname{Min}_{t h}^{k}$ and the maximum threshold $M a x_{t h}^{k}$.

When the average queue length is less than $\operatorname{Min}_{t h}^{k}$, there is no congestion in the node and incoming packets are accepted into the queue. On the other hand, when the average queue size is greater than $\operatorname{Max}_{t h}^{k}$, there is severe congestion at the node and therefore incoming packets are marked. When the average queue size is between $\operatorname{Min}_{t h}^{k}$ and $\operatorname{Max}_{t h}^{k}$, congestion is building up, and incoming packets are dropped depending on the probability of congestion. The placement of $\operatorname{Min}_{t h}^{k}$ and $M a x_{t h}^{k}$ values as seen in the RED queue is shown in Figure 3.7. Using this technique, congestion is detected prior to packet loss. Moreover, for wireless sensor networks, RED is energy efficient as it avoids detection of congestion after a packet has already been dropped, thus reducing the number of re-transmissions.

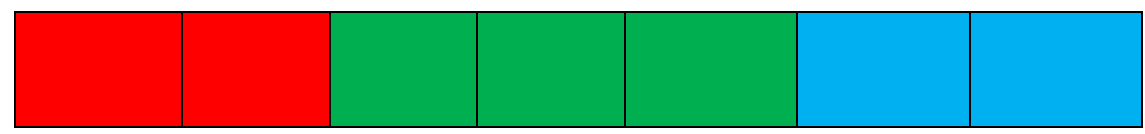

Figure 3.7: Threshold values in a RED queue

According to the RED [46] algorithm, the two threshold values are randomly specified, as long as they are within the maximum buffer size. However, for bursty traffic, the $\operatorname{Min}_{t h}^{k}$ should be high enough to maintain acceptable link utilization. On the other hand, $M a x_{t h}^{k}$ is set depending on the delay that can be tolerated at the node.

In order to overcome the weaknesses of RED stated in [48], PRRP uses an Adaptive RED (A-RED) technique to detect congestion at the nodes. The proposed A-RED used in PRRP determines the $\operatorname{Min}_{t h}^{k}$ and $M a x_{t h}^{k}$ automatically depending on the network's condition. The 
outgoing rate $\mu^{k(i)}$, the incoming rate $\gamma^{k(i)}$ and the queue size $q^{k(i)}$ are the network's parameters used to automatically set the threshold values at node $i$.

\subsubsection{Determining the $\operatorname{Min}_{t h}^{k}$ and $M a x_{t h}^{k}$}

In PRRP, the threshold values are set depending on the outgoing rate $\mu^{k(i)}$, the incoming rate $\gamma^{k(i)}$ and the queue size $q^{k(i)}$ as shown in equation 3.2 and equation 3.3.

\section{i. Determining the minimum threshold $\operatorname{Min}_{t h}^{k}$ :}

$\operatorname{Min}_{t h}^{k}=\frac{\mu^{k(i)}}{\max \left(\gamma^{k(i)}\right)} \times q^{k(i)}$

Where $\mu^{k(i)}$ is the outgoing rate for queue $k$ at node $i$,

$\gamma^{k(i)}$ is the incoming rate for queue $k$ at node $i$ and

$q^{k(i)}$ is the queue size for queue $k$ at node $i$

ii. Determining the maximum threshold $\operatorname{Max}_{t h}^{k}$ :

$$
\operatorname{Max}_{t h}^{k}=\frac{\mu^{k(i)}}{\min \left(\gamma^{k(i)}\right)} \times q^{k(i)}
$$

\section{iii. Congestion detection using A-RED parameters}

The $\operatorname{Max}_{t h}^{k}$ and $\operatorname{Min}_{t h}^{k}$ thresholds in equations 3.2 and 3.3 are then used to detect congestion. Before we discuss congestion detection in PRRP, the congestion index $I^{k(i)}$, at node $i$ and queue $k$ is defined using the threshold values as follows:

$$
I^{k(i)}=\left\{\begin{array}{rlrl}
o, & q^{k(i)} & \leq \operatorname{Min}_{t h}^{k(i)} \\
\frac{q^{k(i)}-\operatorname{Min}_{t h}^{k(i)}}{\operatorname{Max}_{t h}^{k(i)}-\operatorname{Min}_{t h}^{k(i)}}, & \operatorname{Min}_{t h}^{k(i)} \leq q^{k(i)} \leq \operatorname{Max}_{t h}^{k(i)} \\
1, & q^{k(i)} \geq \operatorname{Max}_{t h}^{k(i)}
\end{array}\right.
$$

where $q^{k(i)}$ is the average queue size of queue $k$ of node $i$ and $\operatorname{Min}_{t h}^{k(i)}$ is the minimum threshold of queue $\mathrm{k}$ at node $\mathrm{i}$.

When $q^{k(i)}$ is less than $\operatorname{Min}_{t h}^{k(i)}$, there is no congestion in the node and incoming packets are queued. The congestion index $I^{k(i)}$ is further set to zero, while when the average 
queue size $q^{k(i)}$ is greater than $\operatorname{Max}_{t h}^{k(i)}$, there is severe congestion and incoming packets will not be allowed to queue. In this case, the congestion index $I^{k(i)}$ is set to 1 . When the average queue length is between $\operatorname{Min}_{t h}^{k(i)}$ and $\operatorname{Max}_{t h}^{k(i)}$ a random value $P$ is calculated that satisfies the condition $\operatorname{Min}_{t h}^{k(i)}<P<\operatorname{Max} t_{t h}^{k(i)}$.

Since the $\operatorname{Max}_{t h}^{k(i)}$ and $\operatorname{Min}_{t h}^{k(i)}$ values might create a high variability in the behaviour of the queue, our congestion detection includes a smooth moving average of the two parameters, thus:

$$
A v g \operatorname{Min}_{t h}^{k(i)}=0.9 \times A v g \operatorname{Min}_{t h}^{k(i)}+0.1 \frac{\mu^{k(i)}}{\left.\max \left(\gamma^{k(i)}\right)\right)} \times q^{k(i)}
$$

And

$$
A v g \operatorname{Max}_{t h}^{k(i)}=0.9 \times A v g M a x_{t h}^{k(i)}+0.1 \frac{\mu^{k(i)}}{\left.\min \left(\gamma^{k(i)}\right)\right)} \times q^{k(i)}
$$

The output of the congestion index will be sent to the congestion notification unit so that the other nodes in the network can be notified of the level of congestion at the nodes. This will result in the nodes adjusting their transmission rates depending on the level of congestion, as discussed in Section 3.3.4.

\subsubsection{Congestion Notification}

During congestion, all the active and inactive nodes in the WMSN need to be informed about the presence of congestion in order to avoid packet loss and queueing delay at the buffer.

In PRRP, the nodes are notified about congestion using implicit congestion notification whereby congestion notification is piggybacked in the packet header. Implicit congestion notification is an energy efficient method because it does not contribute to the traffic in the network as in the case of explicit notification. The congestion notification header used takes advantage of the broadcast nature of the wireless medium since all the connected nodes in the network will overhear the congestion notification.

A congestion notification message consists of the following information: the node's average congestion index, the number of child nodes, the node priority $\left(N P^{(i)}\right)$ and the total priority of the node $\left(T P^{(i)}\right)$. The node priority and the total priority are discussed in the next section. Upon overhearing the congestion notification, source nodes will adjust their 
transmission rates using exact rate adjustments while bearing the priority of the traffic and nodes in mind. The PRRP rate adjustment algorithm is described in the next section.

\subsubsection{PRRP Rate Adjustment Algorithm}

PRRP operates in both parent and child nodes in the network topology. Every node is assigned a node priority that corresponds to the importance level of the traffic it generates and the geographic priority of the node. However, since the results presented in this research were generated from a simulation, it was difficult to give a specific meaning to the location of nodes. Thus, the geographic priority of nodes was set equal to the node identification number.

PRRP aims at adjusting the transmission rates of the nodes by considering the nodes' priorities. The initial transmission rates are calculated by the sink nodes which assign the rates in such a way that the nodes with the higher priorities will receive more bandwidth and those with less priority receive less bandwidth. When congestion occurs, each node re-adjusts its transmission rate based on the new rate populated by the sink node or their parent nodes. Still, the distribution of the new rate is done in a way that high priority nodes get the largest portion of the bandwidth.

Before explaining how the PRRP algorithm operates, below are the basic notations that are used:

\section{a) Traffic Priority $-T P^{(i)}$}

The source priority is referring to the priority assigned to node $i$ 's traffic classes. Each node may generate and transmit traffic that belongs to one of the traffic classes (HP-RT, HPNRT, MP-NRT or LP-NRT) and relay transit traffic from child nodes. Let $S P_{(j)}^{(i)}$ denote the priority of the traffic class $j$ generated by sensor node $i$. The weight assigned to traffic class $j$ is set depending on the bandwidth or delay requirement of the traffic, HP-RT traffic is assigned the largest weight while LP-NRT traffic gets the lowest weight.

For each node $i$, the traffic priority $T P^{(i)}$ assigned to traffic generated by the node is obtained as follows:

$$
T P^{(i)}=\sum_{j} S P_{(j)}^{(i)}
$$

where $j$ is the traffic class, for instance $j \in\{$ HP-RT, HP-NRT, MP-NRT or LP-NRT $\}$. 


\section{b) Node Priority $-N P^{(i)}$}

Since the nodes may differ in importance, determined by their location in the sensor network, geographic priority $G e o P^{(i)}$ is also considered. Geographical priorities of nodes will enable sensor nodes to get priority-dependent throughput and is set by the network engineer depending on their requirements.

The total priority of a node $i$ considering its geographic location is obtained as the product of the traffic priority $T P^{(i)}$ and the geographic priority $G e o P^{(i)}$ using equation 3.8.

$$
N P^{(i)}=T P^{(i)} * G e o P^{(i)}
$$

\section{c) Global Priority-GP(i)}

A parent node does not only transmit its own traffic but also relays transit traffic from its child nodes. This should be taken into account when regulating the rate. To capture the transmission rate of the child nodes from their parent, PRRP makes use of the global priority of node $i$. The global priority $G P^{(i)}$ is the sum of $N P^{(i)}$ for all the child nodes of node $i$. It is computed as shown in the equation below:

$$
G P^{(i)}=\sum_{j \in C(i)} G P^{(j)}+N P^{(i)}
$$

Global priorities are only considered for nodes that have child nodes or else will be equal to $N P^{(i)} . G P^{(i)}$ which is used to assign rates to parent nodes and child nodes as will be shown in the next section.

\subsubsection{Computing the nodes' rates at start-up}

\section{Step 1: Determine the sink's output rate}

Using equation 3.9 above, the following section will show how PRRP assigns prioritybased rates to the nodes. Firstly, the sink output rate that is distributed among the nodes is determined by using equation 3.10 :

$$
r^{\operatorname{sink}}=\frac{1}{\operatorname{Avg} T_{S}}
$$

where $A v g T_{S}$ represents the average service time of a packet at the sink.

The average service time is the amount of time it takes to serve a packet. The service time $T_{S}$ of a packets is counted from the time a packet arrives at a node until the time the last 
bit of the packet leaves the node. The service time may vary for each packet because it is affected by congestion as well as the amount of traffic found at a node.

The average service time $\operatorname{Avg} T_{S}$ of the sink node at the instant time $s$ is determined using the exponential weighted moving average (EWMA) formula as follows:

$$
A v g T_{s}=(1-\alpha) A v g T_{s-1}+\alpha \times T_{s}
$$

where $\alpha$ is a positive constant coefficient chosen such that $0 \leq \alpha \leq 1$. In this research, we used a value of $\alpha=0.1$.

\section{$\underline{\text { Step 2: Determine the parent and child nodes' initial rate }}$}

After the sink's output rate is determined, the sink calculates initial rates $r_{\text {initial }}^{(i)}$ for each parent node $i$ using equation 3.12 .

$r_{\text {initial }}^{(i)}=r^{\operatorname{sink}} \times \frac{G P^{(i)}}{G P^{\text {sink }}}$

Equation 3.12 should be repeated to ensure that each node is assigned a rate. Thus, the parent nodes will further compute the child nodes' rates using the rates they have been allocated. For a parent $k$, its child nodes' rates $r_{\text {child }}^{k}$ are computed using equation 3.13.

$$
r_{\text {child }}^{(k)}=r_{\text {initial }}^{(k)} \times \frac{G P^{\text {child }}}{G P^{k}}
$$

\section{Step 3: Determine the priority queue rates}

In order to assign congestion dependant rates at the nodes, the congestion index provided in equation 3.4 is considered. The average congestion index gives an indication of the congestion state of a queue and is used to indicate how much that specific queue contributes to the overall congestion of the node. The average congestion index $A v g I^{q(i)}$ of queue $q$ of child node $i$ is determined as follows:

$$
\operatorname{Avg} I^{q(i)}=\frac{\sum_{j=1}^{N_{i}} I^{j(i)}-I^{q(i)}}{N_{i-1} \times \sum_{j=1}^{N_{i}} I^{j(i)}}
$$


where $N_{i}$ represent the number of queues at node $i$. The weight of the $q$-th queue used by the WRR scheduler is then computed using the average index of each particular queue as follows:

$$
w_{i}^{q}=\frac{p_{i}^{q} \operatorname{AvgI^{q(i)}}}{\sum_{j=1}^{N_{i}} p_{i}^{j} \operatorname{AvgI^{q(i)}}}
$$

where

$$
p_{i}^{1}=\frac{N P(i)}{G P(i)}, p_{i}^{q}=\frac{G P(q)}{G P(i)}, q=1,2,3, \ldots N_{i}
$$

Using the equations above, the rate for each queue $q$ of a child node $i$ is obtained as follows:

$r_{i}^{q}=w_{i}^{q} \times r_{i}$

In equation 3.16, the $S P_{(j)}^{(i)}$ used in calculating $N P^{(i)}$ and $G P^{(j)}$ can be set manually to achieve service differentiation, for example for real-time traffic to get more resources from the network, the $S P_{(j)}^{(i)}$ parameter should be set high enough to distinguish it among other, competing traffic. Chapter 4, Section 4.5.3, discusses how $S P_{(j)}^{(i)}$ is set in our implementation.

\subsubsection{Updating the nodes' rates}

Step 4: Updating the sink, parent and child nodes' rates,

a) The sink and parent nodes will compute new rates for their child nodes at specified intervals by using the output rates of their respective child nodes. For example, a parent node $i$ calculates its new rates $r_{\text {in }}^{i}$ as follows:

$$
r_{i n}^{i}=\sum_{j \in C(i)} r_{o u t}^{j}
$$

where $r_{\text {out }}^{j}$ is the output rate of its $j$ child nodes.

b) The parent nodes compute the difference in rate as follows:

$\Delta r^{i}=\beta \times r_{\text {out }}^{i}-r_{\text {in }}^{i}$

where $\beta$ is a constant close to 1 .

c) Parent $i$ will notify its child $j$ node of new transmission rates as follows:

$$
r_{\text {out }}^{j}=r_{\text {out }}^{j}+\Delta r^{i} * \frac{G P^{j}}{G P^{i}}
$$


d) The new rates for each queue of the nodes will be re-assigned using equation 3.17.

\subsubsection{Multi-path Routing}

PRRP also includes a feature to support multi-path routing as discussed earlier in this chapter. The multi-path routing extends the lifetime of the sensor network by considering the energy of the nodes when upstream traffic is sent. The rules guiding how to choose the correct parent nodes to route traffic consider both the energy of the nodes and the transmission rate. At start-up, a child node with multiple parents will route its data through a parent node that offers the highest transmission rate because all parent nodes are assumed to have the same energy level. During steady state however, parent nodes have depleted part of their energy and the transmission rate allocated by parent nodes to child nodes changes over time. Thus, it becomes difficult to select the parent node that should route data for a given child node. Our proposed algorithm addresses the issue by comparing both the energy level of parent nodes and their output rates, and based on this, decide which parent should transmit the data of the child node. 
The algorithm in Figure 3.8 depicts one way of implementing multi-path routing in a wireless sensor network.

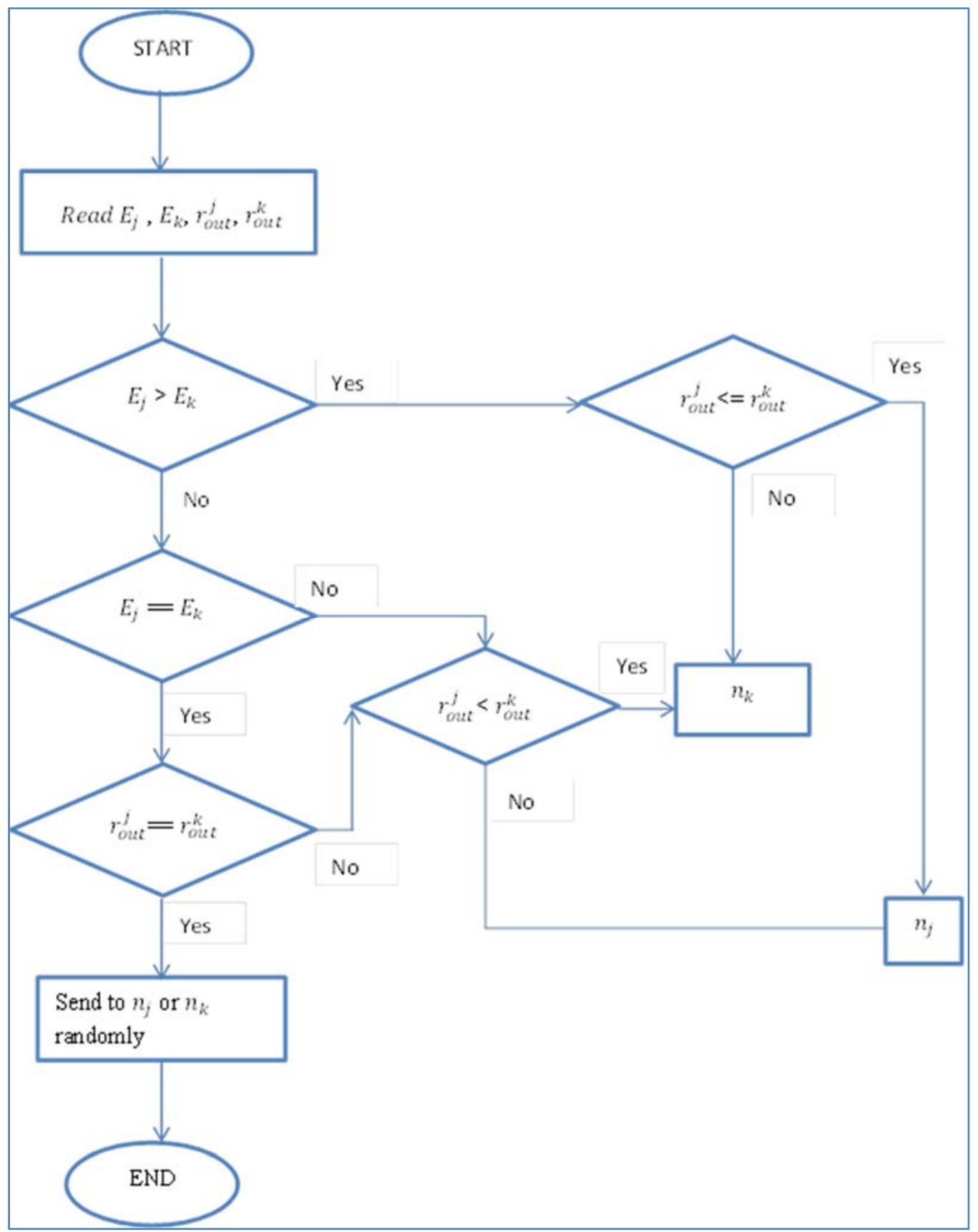

Figure 3.8: Multi-path Routing Algorithm 


\subsection{Chapter Summary}

This chapter outlined the stages involved in the PRRP congestion control protocol. A description of how each stage contributes to congestion control was further outlined, discussing how PRRP can reduce congestion in sensor network. Since the PRRP is specifically implemented to operate in WMSNs, the sensor network that was considered and the architecture of the nodes were also discussed. Our contribution lies in the congestion detection stage as well as in multi-path routing where the energy levels of the nodes are considered before traffic can be transmitted to a certain upstream node. To test the effectiveness of PRRP, simulations were carried out which are discussed in the next chapter. 


\section{CHAPTER 4}

\section{SIMULATION APPROACH, RESULTS AND DISCUSSION}

The previous chapter discussed the design of the proposed protocol, outlined how service differentiation is implemented in PRRP, and provided one way to control congestion in wireless multimedia sensor networks. An efficient congestion control protocol needs to take the challenges of wireless multimedia sensor network into consideration while satisfying the application needs of real-time data. In order to test the effectiveness of the proposed protocol, simulations were performed and the results are presented in this chapter.

\subsection{Introduction}

This chapter starts by discussing the simulation software and parameters used to test the performance of the PRRP protocol. The PRRP protocol is aimed at improving the existing PBRC-SD protocol; therefore the obtained results are compared against PBRC-SD, CCF and PCCP, which are recognised rate-based protocols.

\subsection{Experimental Tool}

Simulations are an effective way of modelling complex protocols and architectures in communication networks since the network performance can be analysed. All simulators follow the cycle: problem definition, build model, execute simulation, analyse results and make decisions [49]. This cycle is also followed in the generation of PRRP results.

A discrete event simulation software using $\mathrm{C}++$ was implemented to test the effectiveness of the proposed protocol. $\mathrm{C}++$ is the suitable language because of its multiparadigm and object oriented nature, which makes it suitable in the design of simulation software [50]. Other simulation software used to test performance for such kind of research problems are NS-2 [51], NS-3 [52], OMnet++ [53] and OPNET [49]. All these simulators have been widely used by researchers to predict how network protocols, applications and architectures behave. NS-2 was developed by UC Berkley and it uses two languages, namely, $\mathrm{C}++$ programming language and $\mathrm{OTcl}$. The two languages perform different tasks: $\mathrm{C}++$ is responsible for ensuring that the applications and protocols designed by OTcl do not take 
excessive time during processing of events and packets. On the other hand, OTcl defines the network layout.

NS-3 is an open source discrete event simulator developed to support features that are not provided by NS-2, such as support for virtualization, software integration, new software core and test bed integration [52]. NS-3 is continually growing and newer modules such as [54] [55][56] are constantly being developed and integrated in the NS-3 code to extend its capabilities. Continuous development in NS-3 modules will enable it to simulate large-scale and complicated networks, rendering it to be the preferred network simulator by researchers. Unlike NS-2 which uses OTcl and C++, NS-3 does not combine any languages and only uses $\mathrm{C}++$.

OMnet++ which stands for Objective Modular Network Testbed in $\mathrm{C}++$ is a discrete event simulator aimed at supporting large-scale simulation, reducing runtime and being extensible and customizable. OMnet++ is made up of simple modules, system modules and compound modules. Communication between these modules takes place through messages. These messages together with the modules form part of the network description language which is used to create the topology. Topologies can therefore either be created using Network Description (NED) or by using the graphical user interface.

OPNET is another event-based simulator, consisting of three domains, namely network domain, node domain and process domain. The three domains deal with defining the overall network topology, the nodes topology and the interaction between these two.

However, it is sometimes very difficult to understand the interaction between the different components of an implementation when dealing with these complex network simulators, thus to keep our implementation simple, we designed our own simulator to better understand the interaction between different entities of our simulator. Our simulator was implemented using the $\mathrm{C}++$ programming language as mentioned earlier in this section.

\subsection{Performance Evaluation Metrics}

In order to evaluate the performance of the proposed protocol, the following QoS metrics are used: queueing delay, packet loss and normalised throughput. These metrics will confirm the effectiveness of the proposed protocol and also indicate whether the service requirements of multimedia applications have been thoroughly considered during the design of PRRP. A brief discussion of the meaning of these metrics is provided below: 
a. Packet loss: This refers to the number of packets that are dropped by the node buffers as a result of node level congestion. In this dissertation, packet loss is reported in terms of packet loss probability. Packet loss probability is calculated by dividing the number of packets not received at the sink by the number of packets sent by the source as shown in equation 4.1 .

$$
\text { Packet loss probability }=\frac{\text { number of packets not received at the sink }}{\text { number of packets sent by the source }}
$$

b. Throughput: Generally, the bandwidth used to transfer traffic in sensor networks impacts the performance of the network. However, factors such as collision, interference etc., may negatively affect the maximum bandwidth, thus the effective rate at which the traffic is sent is generally below the bandwidth and of more importance as it gives a better idea of the amount of data received at the sink. Throughput is the number of packets that are successfully received at the sink per unit of time. The throughput is calculated as follow:

$$
\text { Throughput }=\frac{\text { Total number of packets received at the sink }}{\text { Simulation time }}
$$

In this research, throughput is reported as the normalised total number of individual nodes' throughput.

c. Packet delay: This represents the time that elapses between the sending of a packet and its receipt by the destination node. Only queueing delay is considered in this research because it is caused by node level congestion. The packet delay is calculated as follows:

$$
\text { Delay }(s)=\text { receiving time of packet }(i) \text { - sending time of packet }(i)
$$

To eliminate the delay caused by the network, real-time applications use a playback buffer at the receiver. However, high packet delay has a negative impact on the playback buffer at the receiver and on the visual quality of multimedia streams, and therefore needs to be kept minimal in the network. 


\subsection{Simulation setup}

PRRP aims at improving PBRC-SD, which is a priority-based rate congestion control protocol. This means that PRRP partially emulates the design and implementation of PBRCSD to achieve congestion control in WMSNs. In order to improve PBRC-SD, three different types of scenario were simulated. Firstly, PBRC-SD's performance was evaluated using the single-tier architecture depicted in Figure 4.1 below. This is the same topology used to evaluate PBRC-SD performance in [41]. Chapter 2 discussed the general operation of a single-tier architecture (see Figure 2.4) and how it applies to WMSNs. Secondly, one additional parent node was added onto the original single-tier topology (see Figure 3.3) used in PBRC-SD, in order to observe how PBRC-SD scales with an additional parent node. Lastly, multi-path routing was introduced in the single-tier topology having the extra parent node (see Figure 3.4). The multi-path routing considers energy when routing as discussed in Chapter 3 Section 3.3.5. In each of these three scenarios, performance was measured in terms of the parameters mentioned in Section 4.3, namely packet loss, packet delay and normalised throughput.

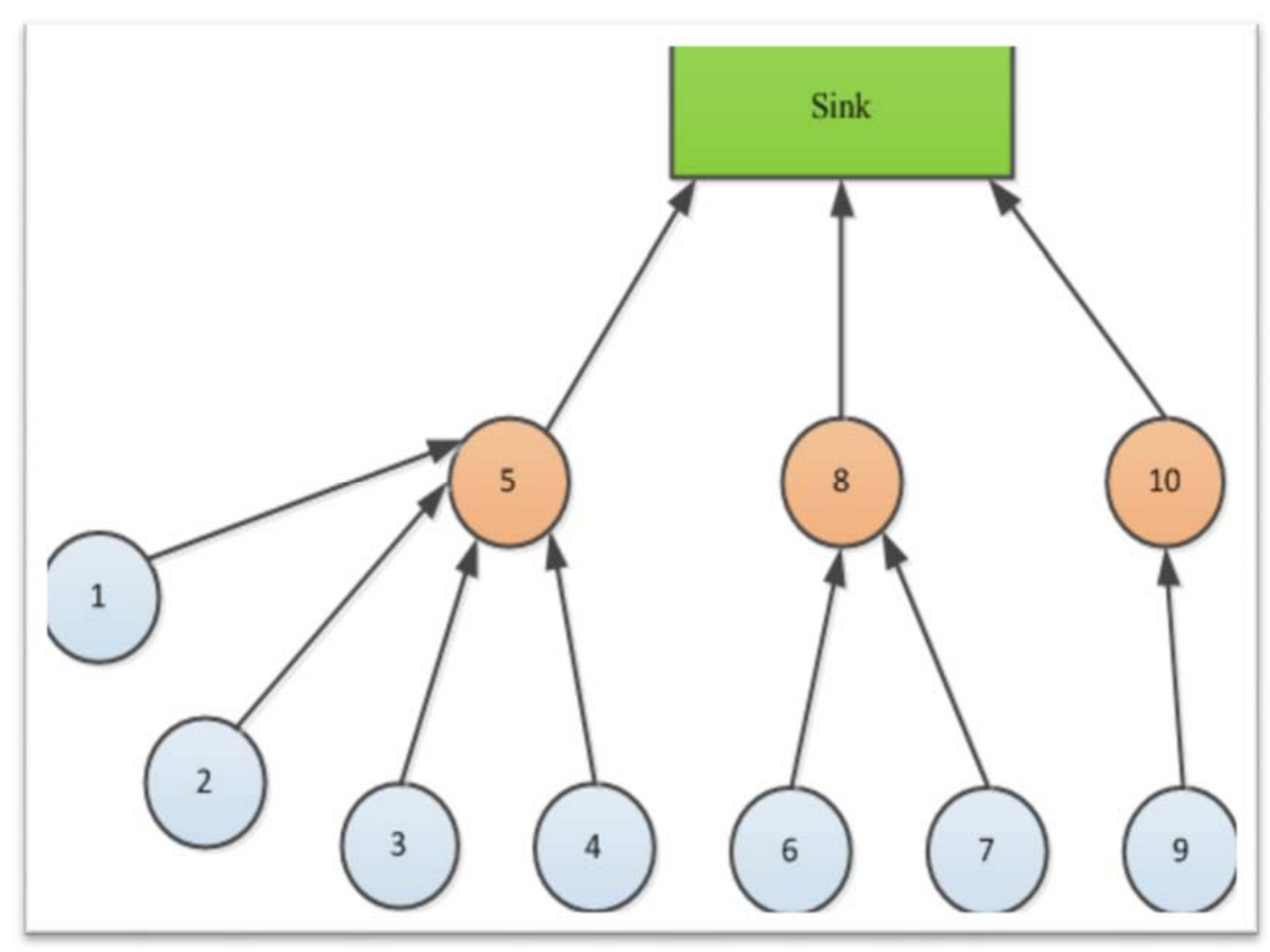

Figure 4.1: Simulated Single-Tier Topology 
In all the topologies used in the simulation, the traffic corresponding to the four traffic classes (HP-RT, HP-NRT, MP-NRT, LP-NRT) were generated by all the nodes. To imitate the traffic characteristics transmitted by the sensor nodes, Exponential Traffic Model (ETM) and Constant Bit Rate (CBR) traffic model were generated. CBR traffic produce fixed sized packets during transmission while ETM created equal sized packets during the "ON" state and did not generate any traffic during the "OFF" state. The time to transmit fixed length packets for ETM was modelled by an exponential random service time while fixed service time was used to transmit CBR traffic.

Table 4.1 indicates the different values of the different parameters used to simulate our priority-based rate protocol.

Table 4.1 Simulation Parameters

\begin{tabular}{|l|l|}
\hline Buffer size & 100 packets \\
\hline Simulation time & 100 seconds \\
\hline Network size & 10 nodes, 11 nodes \\
\hline Service time & 0.001 seconds \\
\hline Transmission range & $35 \mathrm{~m}$ \\
\hline Total Area & $140 \mathrm{~m} * 140 \mathrm{~m}$ \\
\hline Routing type & Single-path, Multi-path \\
\hline
\end{tabular}




\subsection{Simulation Results}

\section{Evaluation of the congestion control algorithm}

Several experiments were studied to evaluate the performance of the congestion control algorithm. In the first experiment, all the nodes have the same priorities (Node Priority $N P^{(i)}$ field is set to the same value, namely 1) and are all actively transmitting traffic. Two types of flow are generated by the nodes, constant bit rate and exponential traffic. The sensor nodes offer two types of services, fixed service and an exponential random service. PBRC-SD protocol is tested using the topologies outlined in Figures 3.4, 4.1 and 3.3.

In the second experiment, the conditions of the first experiment are maintained, but the proposed protocol is tested. The results for both experiments are then compared. For the first experiment, results are obtained for both fixed and random service time while only random service time results are considered in the second experiment. This is because we had observed that there was no change in the fixed service time results. Both experiments use the parameters in Table 4.1.

\subsubsection{PBRC-SD results using Random Early Detection}

\subsubsection{PBRC-SD with Single-Tier Topology results}

In the first experiment, a single-tier topology similar to the one used in [41] was used to validate our implementation. Figures 4.2 and 4.3 below show normalized throughput of CCF, PCCP and PBRC-SD for fixed service time and random service time as found in [41].

\section{i. Constant Bit Rate traffic and Fixed service time}

Simulation results for fixed service time in Figure 4.2 show that the total normalized throughput for CCF, PCCP and PBRC-SD is $0.999,0.968$ and 0.980 respectively. Observation of these results further indicates that the throughput for CCF and PBRC-SD is higher than for PCCP. The high throughput achieved by PBRC-SD is attributed to its rate adjustment algorithm, which assigns rates based on the number of packets in the buffer. Keeping track of the number of packets enables the rate adjustment algorithm to avoid congestion, which may result in packet loss; hence the increase in throughput.

The queueing delay for CCF is $0.001 \mathrm{~s}$, for PCCP is $0.0005 \mathrm{~s}$ while PBRC-SD has achieved a delay of $0.0007 \mathrm{~s}$. The delay results are summarized in Appendix A Table A.2. Since PBRC-SD has not encountered congested nodes, this caused the low delay. In multimedia 
communication, lower delay and lower jitter are desirable as they guarantee that data content will be seen clearly and videos will play without interruption.

Further, all the protocols have zero packet loss probability. Having zero packet loss probability suggests that the protocols have not experienced node level congestion. Section 2.2.2 discussed the effects of packet loss in multimedia communication, therefore achieving a low packet loss will save the resources of the sensor network and will further preserve the visual quality of the images.

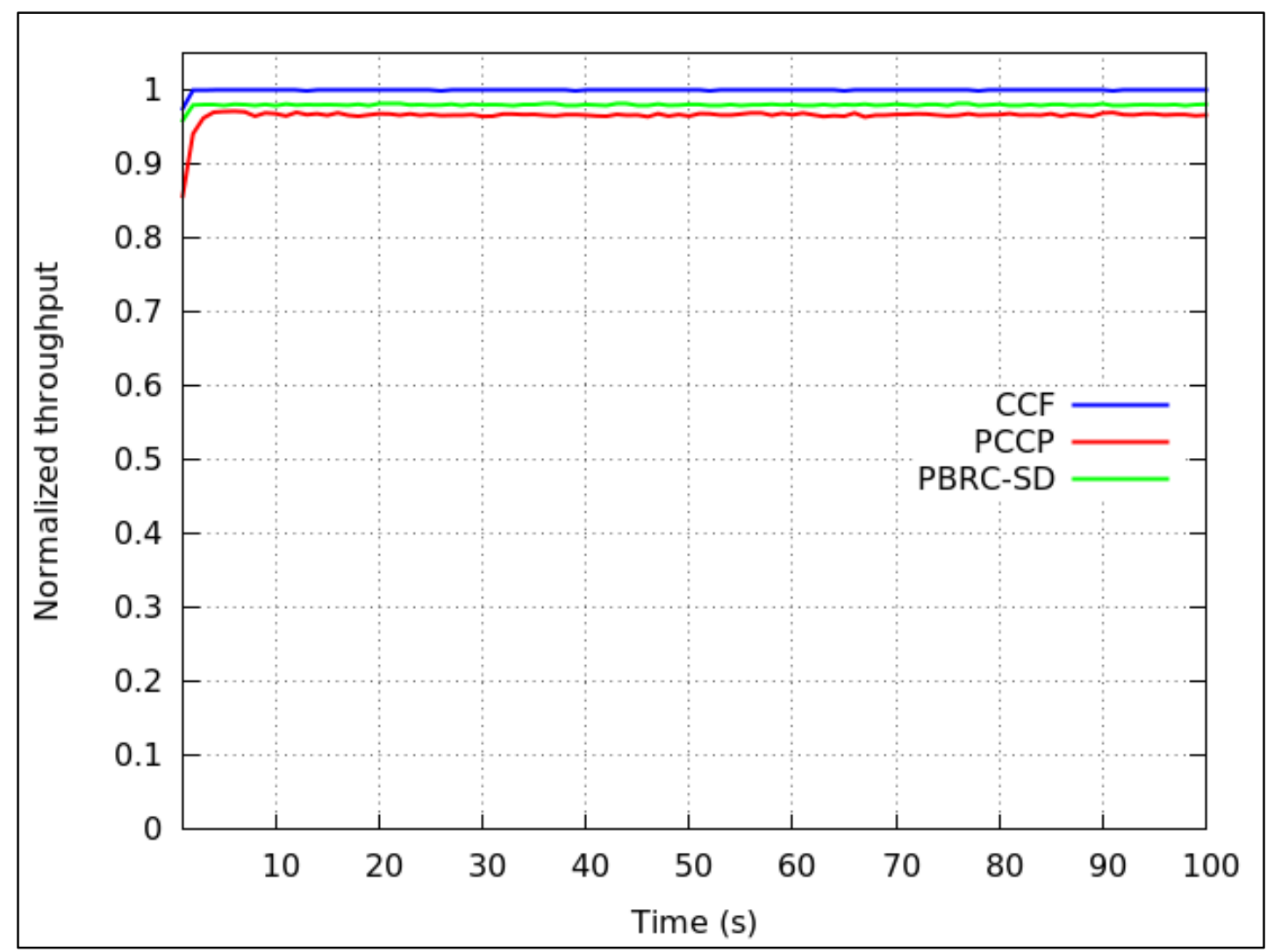

Figure 4.2: Throughput for fixed service time

ii. Exponential traffic and Random service time

Results for random service time as shown in Figure 4.4 show that PCCP has achieved a throughput of 0.826, which is lower than the throughput for CCF and PBRC-SD. CCF has the highest throughput of 0.999 compared to PBRC-SD, which achieved throughput of 0.931 . As for the PCCP protocol, the low throughput is due to the fact that PCCP does not perform very well during random service time as it always tries to maintain lower queue length which 
impacts its throughput. Similar to the fixed service time, PBRC-SD still achieved a high throughput owing to its rate adjustment algorithm that tries to avoid congestion.

Regarding packet loss probability, PBRC-SD has the lowest packet loss probability 0.0005 followed by PCCP with a loss probability of 0.025 and CCF with a loss probability of 0.065, as illustrated in Figure 4.4 a). Lower packet loss probability in PCCP and PBRC-SD is derived from the short queue lengths which both protocols aim to maintain. In addition to this, PBRC-SD uses a similar congestion detection technique as the RED active queue management which tries to avoid congestion in advance, resulting in low packet loss.

In terms of queueing delay, PBRC-SD has the lowest delay of $0.009 \mathrm{~s}$, which is good for real-time applications, while PCCP has a delay of $0.021 \mathrm{~s}$ and CCF has a delay of $0.081 \mathrm{~s}$, which is the highest among all protocols. Although CCF has the highest throughput, some of the received packets may be dropped by the receiver because they have exceeded the playback delay and this may negatively impact on the visual quality of the image. Results therefore show that CCF has both the highest packet loss probability and the highest queueing delay - this is due to requesting nodes to resend the lost packets.

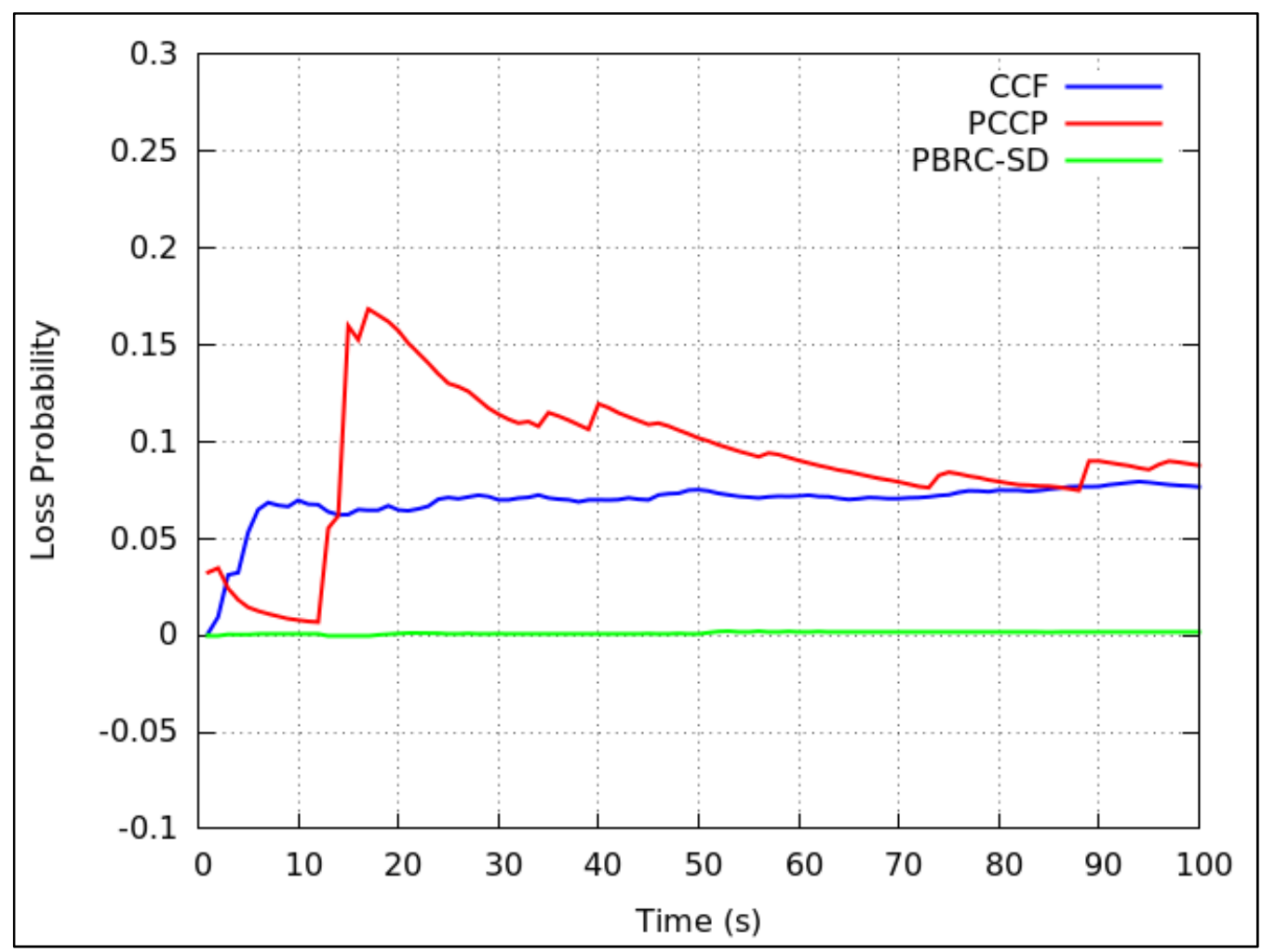

a) Loss probability 


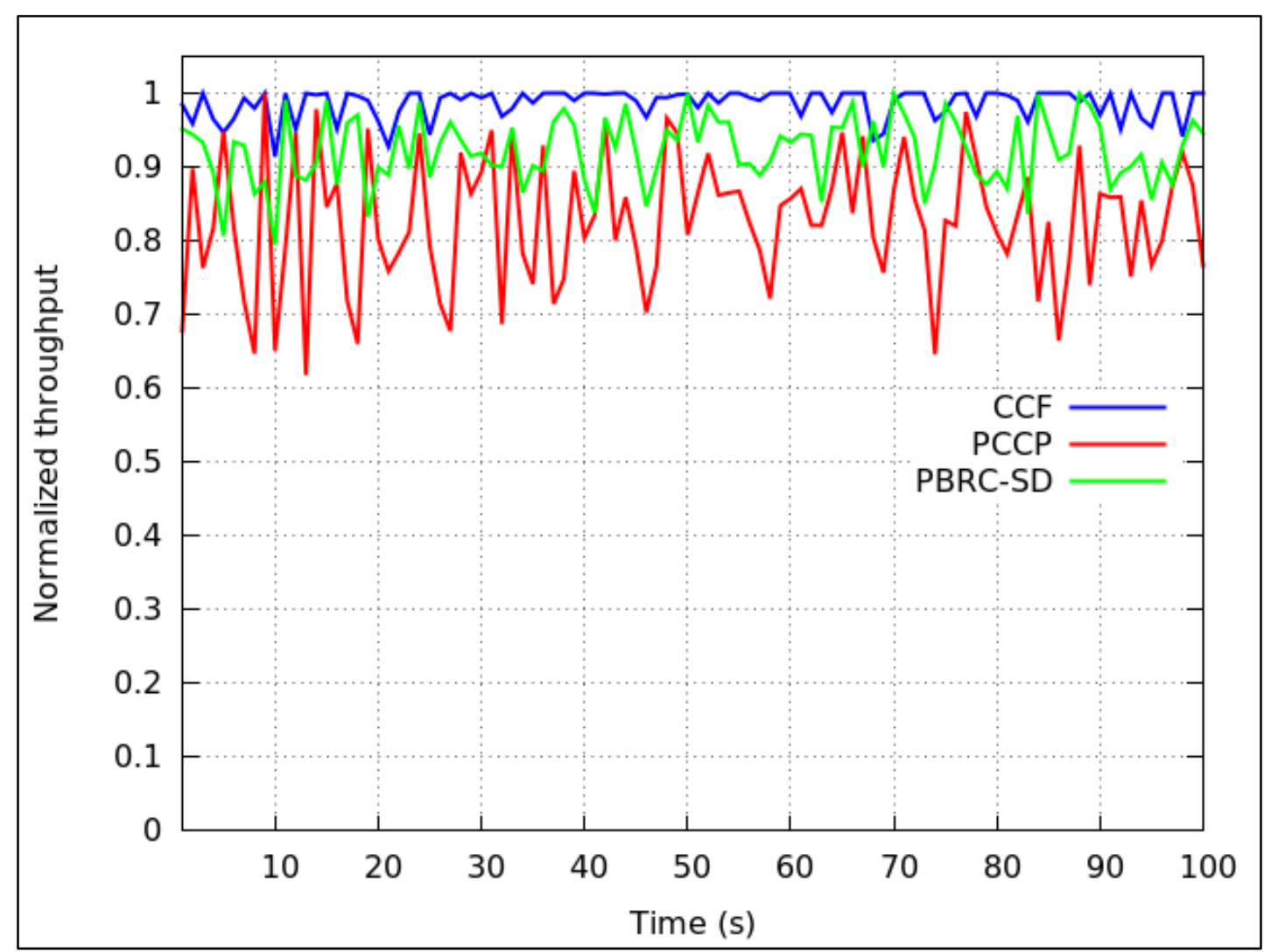

b) Normalized throughput

Figure 4.3: Loss probability and normalized throughput for random service time

In conclusion, the results obtained from this experiment are similar to those obtained in [41], and thus validate the correctness of the implementation of the three priority-based rate protocols, namely PBRC-SD, PCCP and CCF.

\subsubsection{PBRC-SD with Single-Tier with extra parent node results}

In the second experiment, a test on how PBRC-SD adjusts with the change of the network topology was performed. Thus, another additional parent node is added to the original topology as illustrated in Figure 3.3. Parent nodes act as cluster heads and they are normally equipped with more resources than the child nodes. In this research, parent nodes are also responsible for disseminating rate adjustment information to the child nodes which they receive from the sink.

The same simulation parameters as those in Table 4.1 are used and the results from the simulation are discussed below and compared to the results in the previous section. PBRC- 
SD's performance is again compared with CCF and PCCP protocols as was done in the previous scenario.

\section{i) Constant Bit Rate traffic and fixed service time results}

Figure 4.4 a) shows the results for the CBR traffic. From Figure 4.4 a), it is observed that PBRC-SD experiences the highest drop in throughput when an additional parent node is added to the topology. The results show that PBRC-SD's throughput has dropped from 0.980 in the original topology to 0.799 in the topology with the extra parent node. The significant drop in PBRC-SD's throughput is caused by the buildup of packets in the queues, resulting in the buffer occupancy being greater than $\operatorname{Max}_{t h}$ threshold, causing the throughput to drop. Additional support for this weakness of RED parameters is indicated in [57] [47].

In general, when congestion builds up in a network, it can easily result in packet loss and packet delays. However, by using PBRC-SD in WSN, packet loss did not happen due to the fast congestion detection technique implemented in PBRC-SD, which triggers the rate adjustment technique before the network starts dropping packets. This is proven by the packet loss probability being zero for PBRC-SD. Figure $4.5 \mathrm{~b}$ ) illustrates the packet loss results for CCF, PBRC-SD and PCCP.

The delay for PCCP is $0.000526 \mathrm{~s}$, for CCF it is $0.00341 \mathrm{~s}$ and for PBRC-SD it is $0.00338 \mathrm{~s}$ (see Table A.2 Appendix A). Comparing these delay results with those of subsection 4.5.1.1, the delay has dropped for CCF and PBRC-SD protocols and has slightly increased for PCCP. PCCP's delay increased by 5.2\%, while CCF and PBRC-SD decreased by $66 \%$ and $52 \%$ respectively. The increase in PCCP's delay is attributed to the poor technique used in dealing with an increase in packets in the network. PCCP, unlike PBRC-SD, cannot effectively deal with event driven traffic, hence the increase in the delay. The decrease in PBRC-SD's delay is due to the ability of the rate controller which detects congestion before it can occur, thus an increase in packets does not build up at the node buffer. 


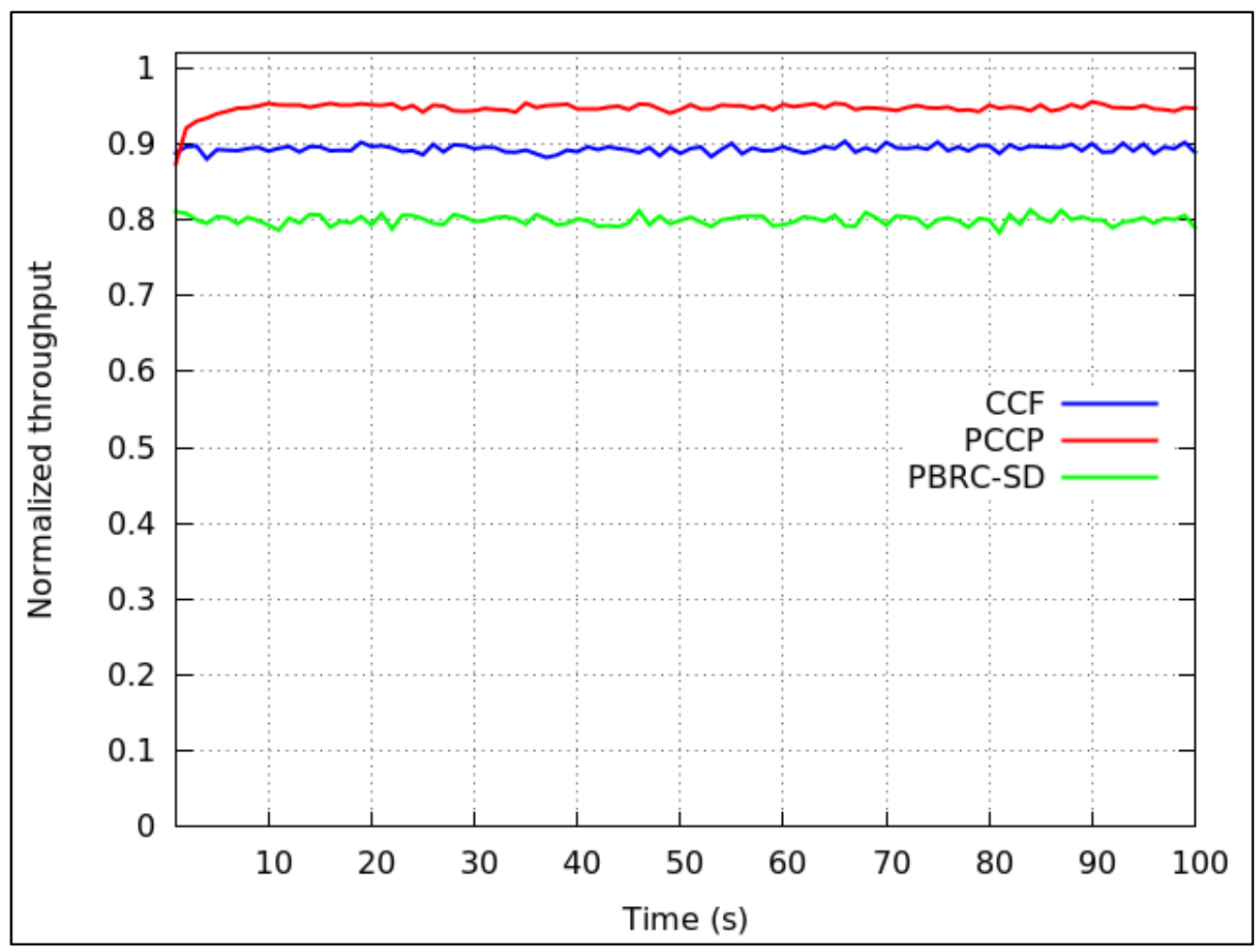

a) Normalized throughput

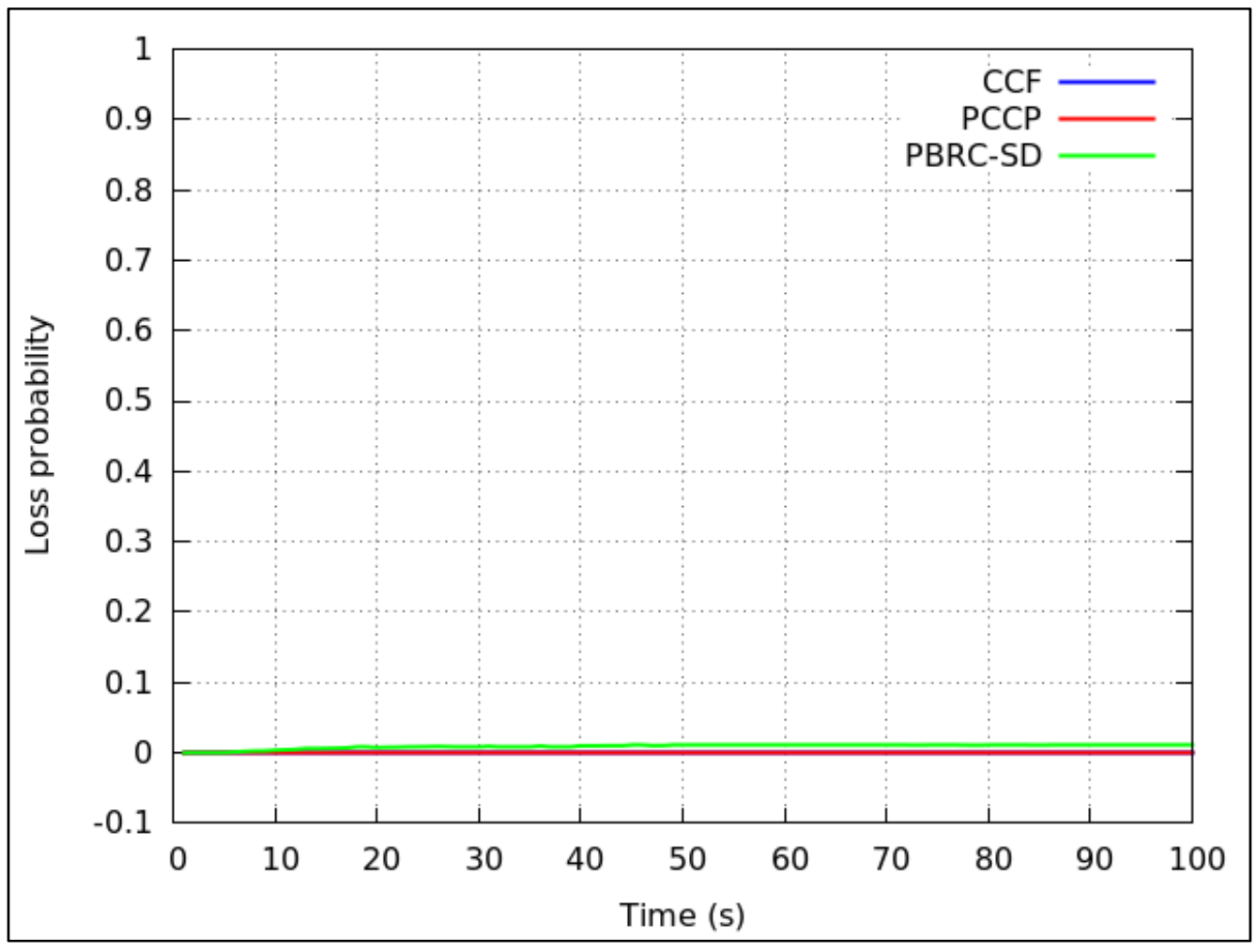

b) Loss probability

Figure 4.4: Normalized throughput and loss probability for fixed service time 


\section{ii) Exponential traffic and Random service time results}

Figure 4.5 a) below depicts the results for random service time during the addition of the extra parent node. The normalized throughput for CCF, PCCP and PBRC-SD is equal to $0.929,0.686$ and 0.824 respectively. Results indicate that the total normalized throughput for all three protocols has decreased, with PCCP having the highest drop of $16.9 \%$ followed by PBRC-SD with a drop of $11 \%$ and CCF a drop of 7\%. Again, this confirms that PCCP does not perform well during random service time. The drop in PBRC-SD's throughput indicates that the rate adjustment algorithm cannot share the rates effectively with the increase in the topology.

The packet loss probability results in Figure 4.5 b) show that CCF and PBRC-SD's loss probability has dropped to zero while PCCP has a packet loss probability of 0.0010 . This is because PCCP tries to maintain small buffer size that may easily get congested when there is a large amount of data to report to the sink.

The delay for PCCP is $0.0087 \mathrm{~s}, 0.0053 \mathrm{~s}$ for CCF and 0.0019s for PBRC-SD (see Table A.5, Appendix A). Compared to the delay results in the case of Section 4.5.1.1, there is a drop of $58 \%$ for PCCP, 93\% for CCF and 78\% for PBRC-SD. The drop in PBRC-SD's delay is attributed to the rate adjustment algorithm, which can handle the increase in packets at the nodes. 


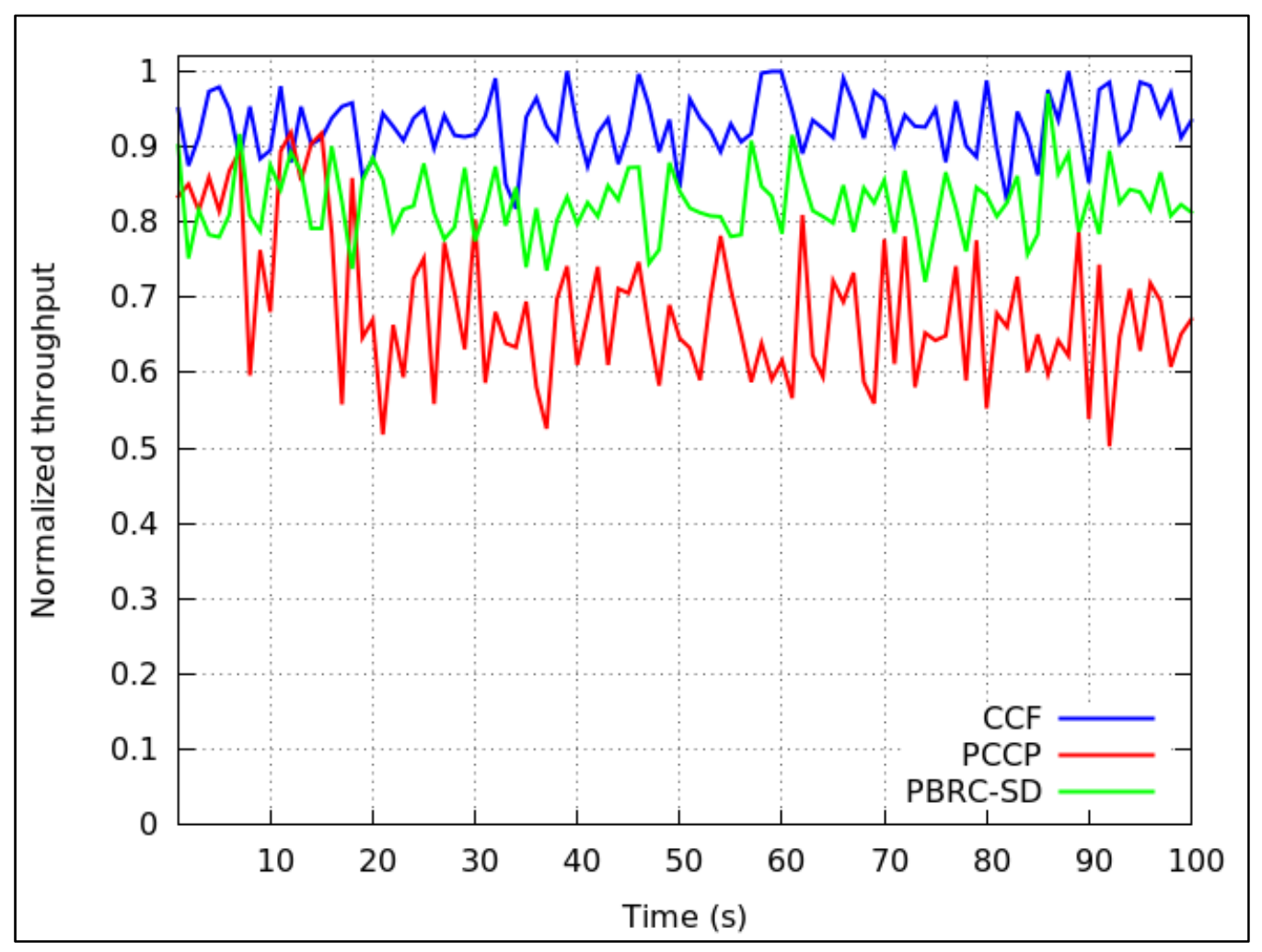

a) Normalized throughput

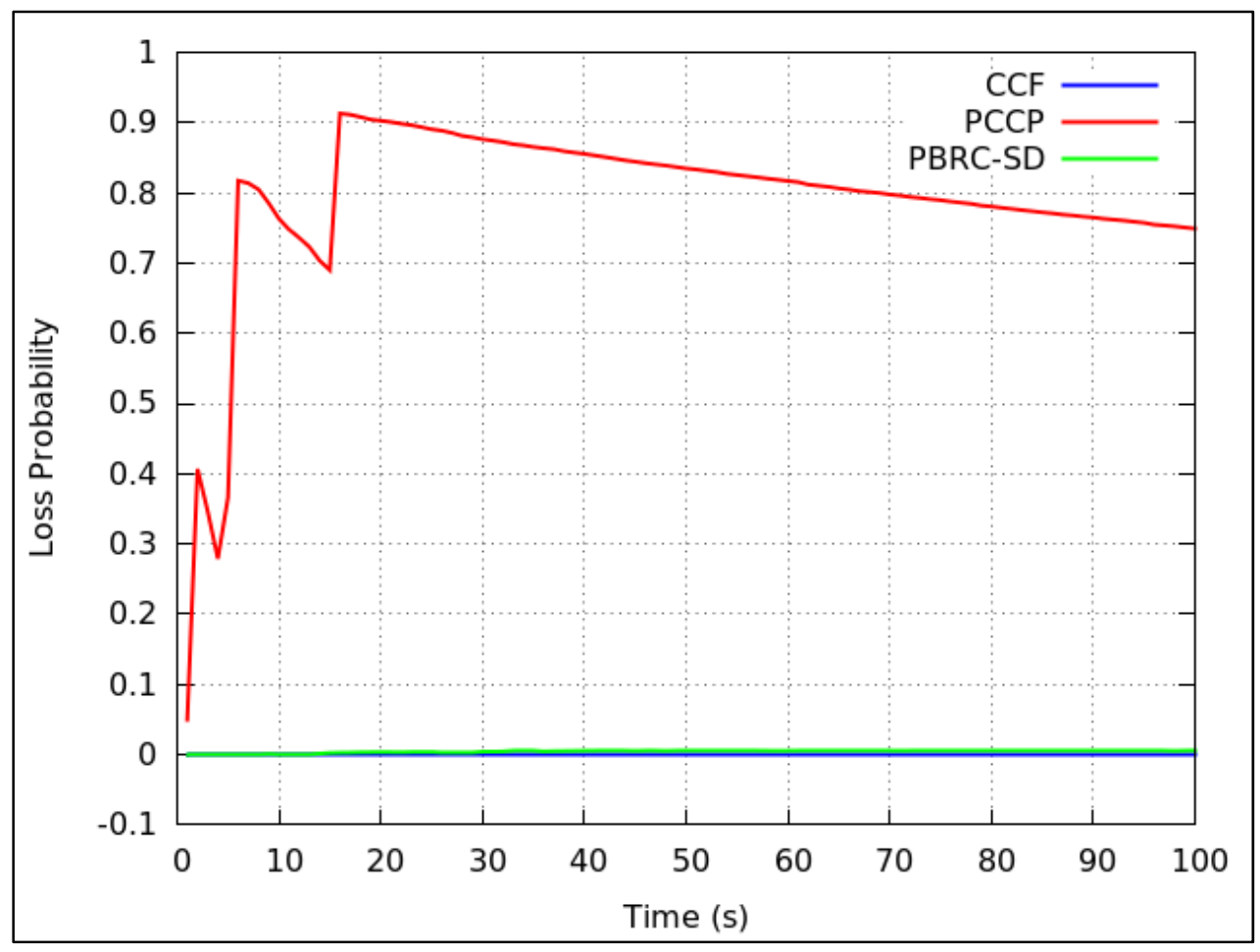

b) Loss probability

Figure 4.5: Normalized throughput and loss probability for random service time 


\subsubsection{PBRC-SD on Multi-path Wireless Sensor Networks}

In this scenario, we evaluated the performance of PBRC-SD on a multi-path WSN topology in terms of delay, total normalized throughput and packet loss probability.

\section{i) Constant Bit Rate traffic and Fixed Service time results}

Firstly, we simulated a multi-path wireless sensor network, where all nodes generate and transmit CBR traffic which are processed by relay nodes using a fixed service time. Figure 4.6 gives the results for the normalized throughput for PCCP, CCF and PBRC-SD. As can be observed from the graphs, the normalized throughput has dropped for all three protocols when compared to results from Section 4.5.1.1 The normalized throughput for PCCP, CCF and PBRC-SD is $0.848,0.905$ and 0.783 respectively. This indicates a drop in throughput of $10 \%$ for PCCP, $10 \%$ for CCF while PBRC-SD has dropped by $25 \%$. Since PBRC-SD does not consider multi-path routing, the rate adjustment caused the unbalanced traffic resulting in throughput dropping.

None of the three protocols experienced packet loss, thus the packet loss probability for all protocols is zero. The delay for all three protocols has decreased (see Table A.2, Appendix A). PCCP has a delay of $0.000408 \mathrm{~s}$, CCF a delay of $0.000302 \mathrm{~s}$ and PBRC-SD a delay of 0.00022 s, showing a decrease of $20 \%, 7 \%$ and $68 \%$ respectively. 


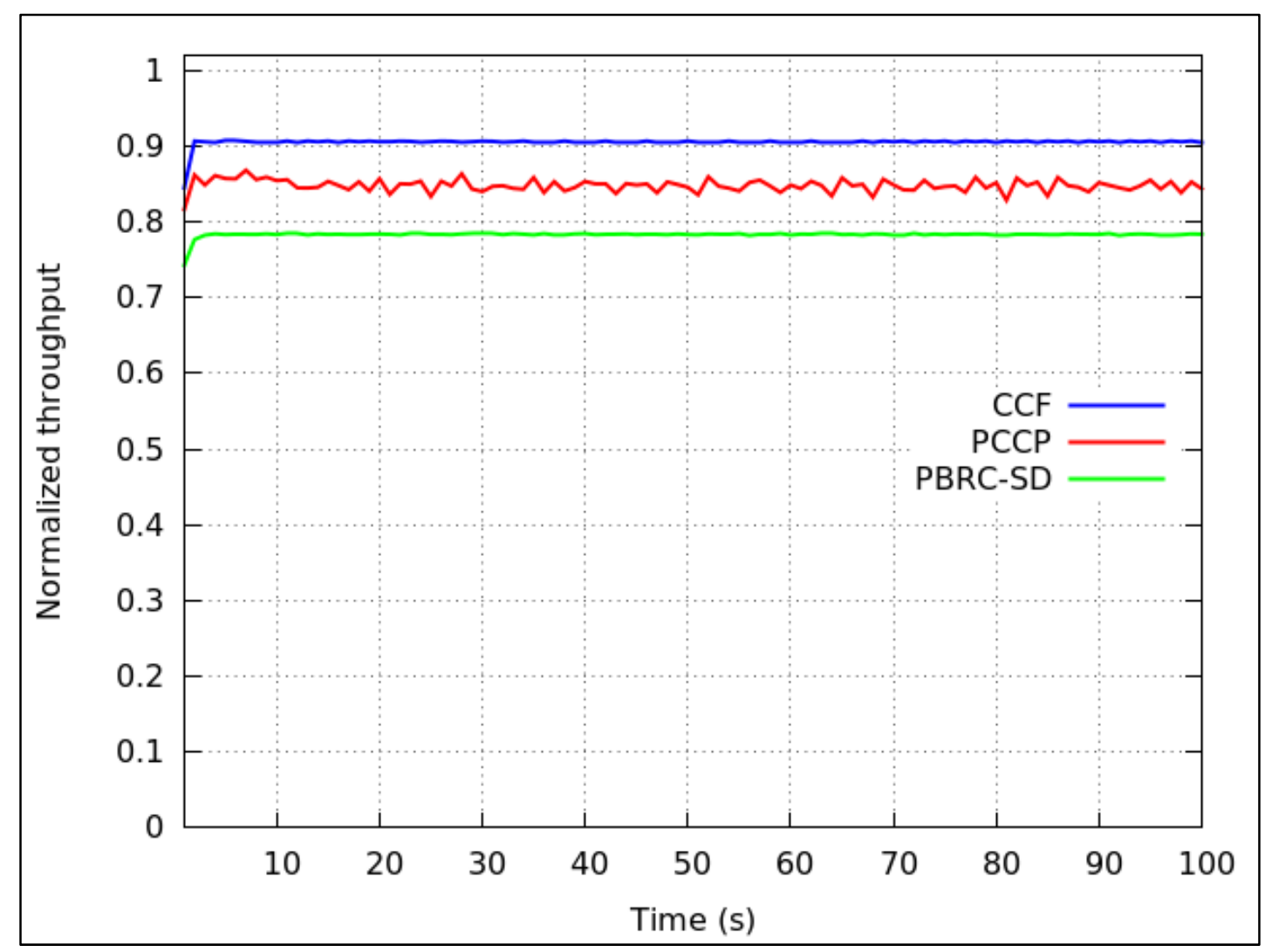

Figure 4.6: Normalized throughput for fixed service time

\section{ii) Exponential traffic and Random Service time results}

During random service time, all the protocols also dropped in throughput as illustrated in Figure 4.7 a). The normalized throughput for PCCP is $0.765,0.948$ for CCF and 0.835 for PBRC-SD. PBRC-SD also encountered the largest drop in throughput owing to the sensitivity of the minimum and maximum RED threshold values used in detecting node level congestion.

The loss probability is zero for CCF, 0.000078 for PCCP and 0.00487 for PBRC-SD as depicted in Figure 4.7 b). PCCP has a slight loss probability caused by the small buffer sizes brought on by its rate adjustment.

Table A.5 in Appendix A summarized the delay experienced by all three protocols. The CCF protocol has the highest delay of $0.0191 \mathrm{~s}$, PBRC-SD protocol has a delay of $0.0100 \mathrm{~s}$ and PCCP achieved the lowest delay of $0.0045 \mathrm{~s}$. The delay in PBRC-SD is caused by another problem of RED whereby the average queue size is closer to the maximum threshold value resulting in higher queuing delay for multimedia traffic. 


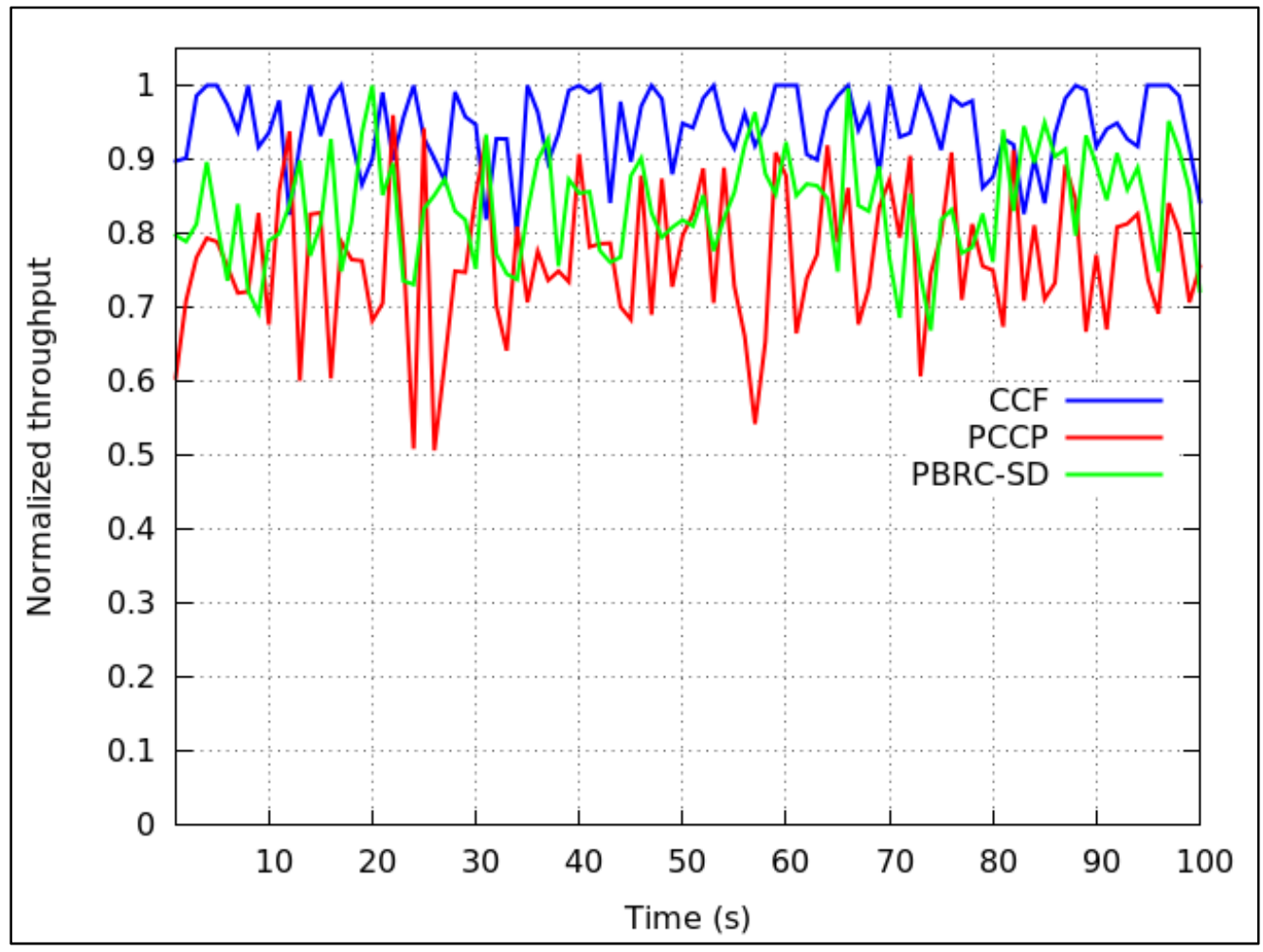

a) Normalized throughput

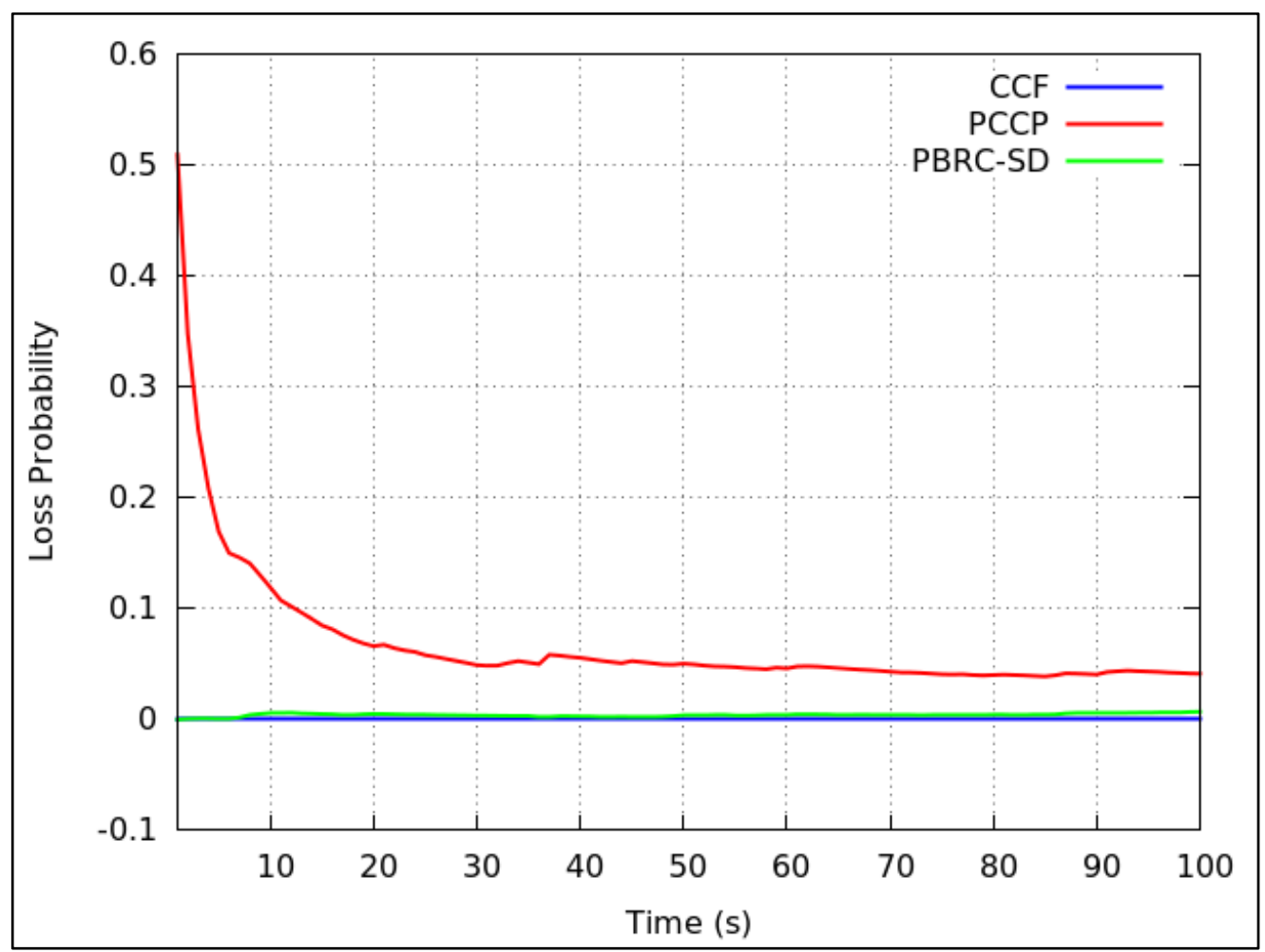

b) Loss probability

Figure 4.7: Normalized throughput and loss probability for random service time 


\subsubsection{PRRP results using Adaptive Random Early Detection}

The simulation results in the last section indicate that PBRC-SD experienced a big drop in throughput, both in the single-tier topology with an extra parent node and when multi-path routes were used to send data. However, for both the multi-path and single-tier with extra parent node topologies, PBRC-SD maintained extremely low packet loss probability and delay.

In order to improve the performance of PBRC-SD in a single-tier with extra parent node or in a multi-path routing WMSN, a new protocol named PRRP was proposed, which included an adaptive RED queue and multi-path routing strategy (see Chapter 3, Section 3.3.5). The performance of PRRP was carried out using the same simulation parameters of the previous section. The results obtained are compared with PCCP and PBRC-SD because they are all considered as priority-rate based protocols.

\subsubsection{PRRP with Single-Tier Topology Results}

\section{i. Exponential traffic and Random Service time results}

Simulation results for random service time are shown in Figure 4.8 and Tables A.6-A.8 in Appendix A. The results show that when A-RED parameters are used, the throughput increases as illustrated in Figure 4.8. Compared with the previous scenario where RED was used (see Section 4.5.1.1), the normalized throughput results for PBRC-SD increased from 0.931 to 0.996 , showing an increment of $6 \%$. The increase in throughput indicates that the PRRP protocol performs well when the threshold values are adjusted automatically depending on the network's conditions, unlike in the case of using static RED parameters.

The loss probability for PBRC-SD increased from 0.0005 to 0.0087 showing that the buffer experienced some congestion which caused packet loss and queueing delay as the PBRC-SD's delay also increased from 0.009 s to $0.0776 \mathrm{~s}$, as shown in Figure $4.8 \mathrm{~b}$ ). 


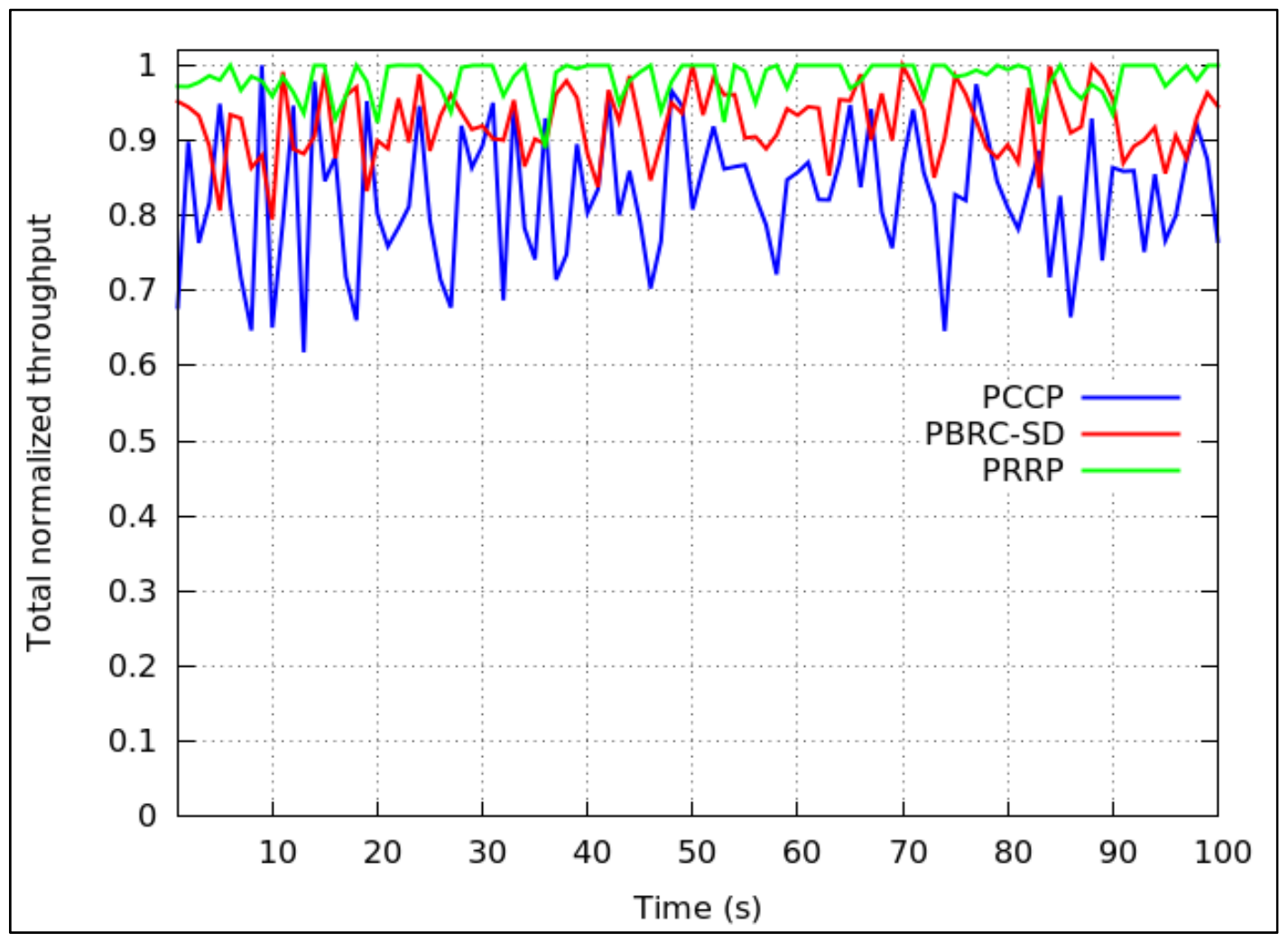

a) Normalized throughput

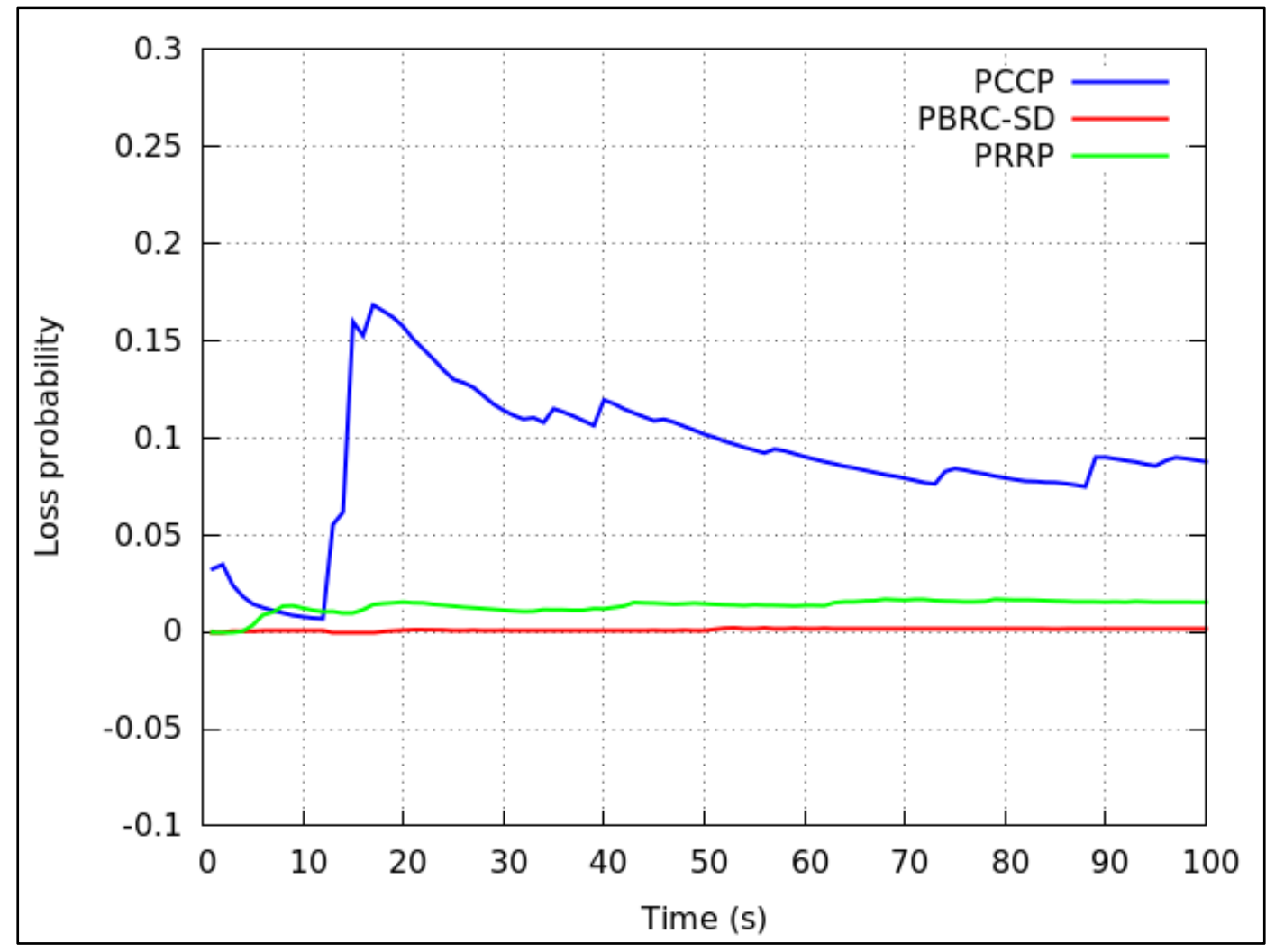

b) Loss probability

Figure 4.8: Normalized throughput and loss probability of random service time 


\subsubsection{PRRP with Single-Tier with extra parent node results}

i. Exponential traffic and Random service time results

The results in Figure 4.9 show the performance of PRRP after the addition of a parent node to the single-tier network of Figure 4.1. The normalized throughput in Figure 4.9 a) shows that PRRP obtains a throughput of 0.880 , an increment of $7 \%$ when compared to PBRC-SD in Section 4.5.1.2. The results also show that PRRP achieves the highest throughput among the three protocols, a good indication that the A-RED is able to maintain an acceptable average queue length that is less than the maximum threshold.

The loss probability in PRRP is zero as illustrated in Figure 4.9 b), which is the same as in the PBRC-SD protocol. This means that both protocols can avoid node level congestion as their queue levels are kept low by the A-RED. However, PCCP encounters a packet loss probability of 0.0010 . The queueing delay for PCCP, PRRP and PBRC-SD protocols are $0.00875 \mathrm{~s}, 0.00412 \mathrm{~s}$ and $0.0019 \mathrm{~s}$ respectively. This is depicted in Table A.8 in Appendix A.

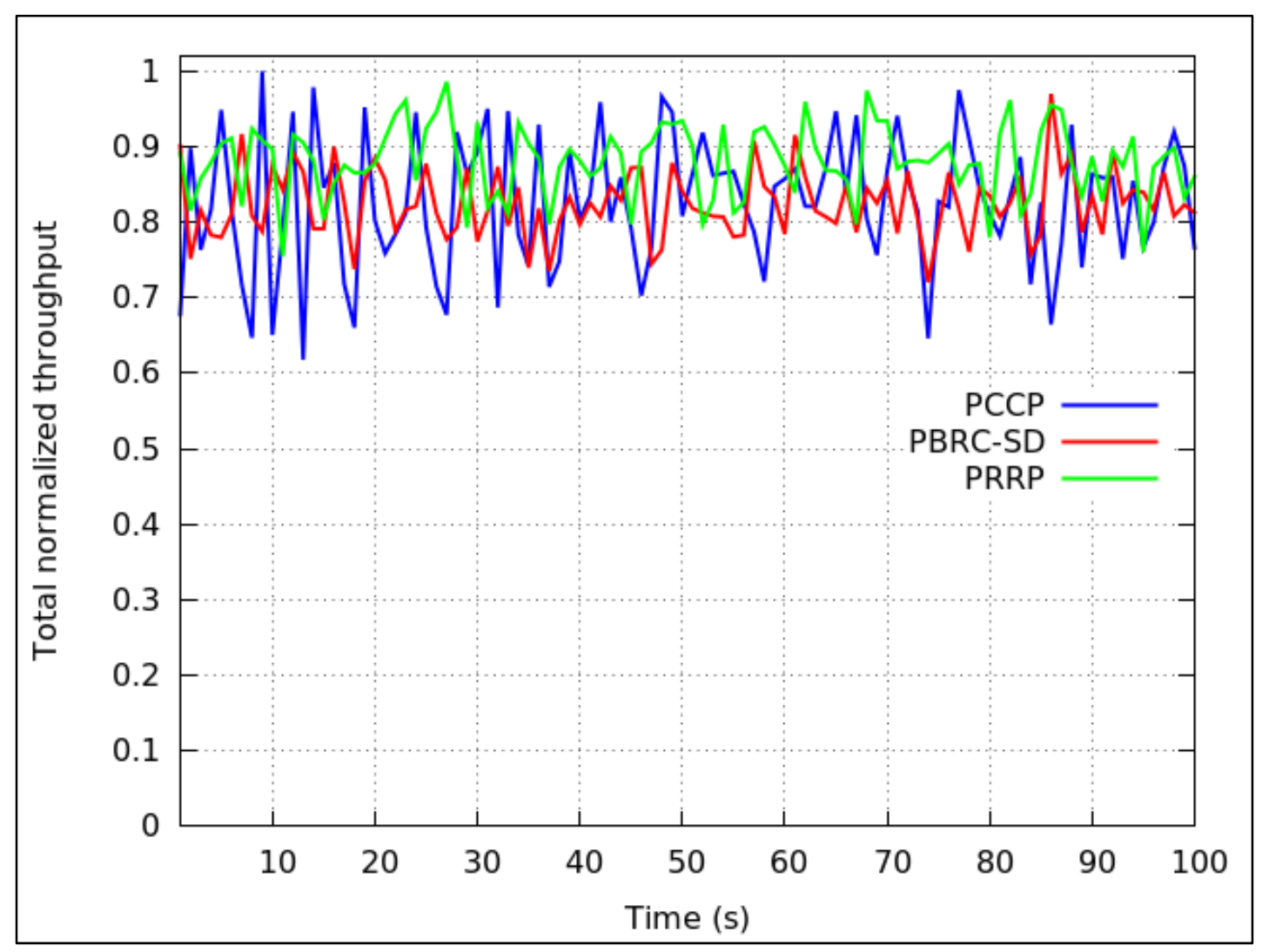

a) Normalized throughput 


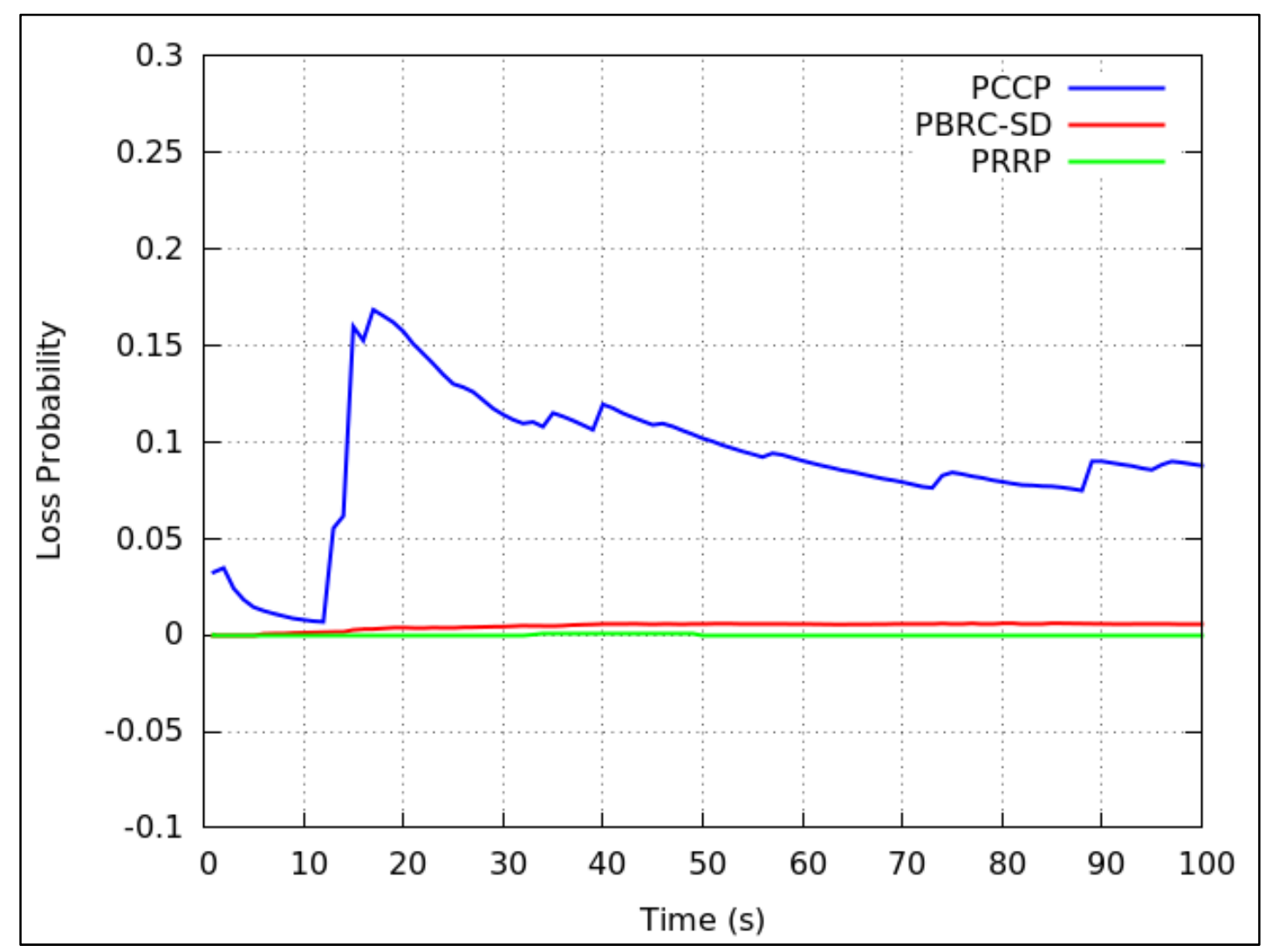

b) Loss probability

Figure 4.9: Normalized throughput and loss probability for random service time

\subsubsection{PRRP on Single-Tier with Multi-path Topology}

i. Exponential traffic and Random service time

In multi-path topology, PRRP obtains a throughput of 0.929 showing an improvement of $11 \%$ when compared to PBRC-SD. PRRP also has the highest throughput when compared with the other protocols as shown in Figure 4.10. This means that the rate adjustment can distribute the traffic load fairly among the other nodes. Multi-path routing has multiple benefits to sensor networks, such as the ability to extend the network lifespan.

The packet loss probability of PRRP is 0.000736 , showing a decrease of $85 \%$ when compared to the PBRC-SD results in Section 4.5.1.3. The PRRP queuing delay is $0.0146 \mathrm{~s}$ which has increased from 0.001s. (Table A.7-A.8, Appendix A). 


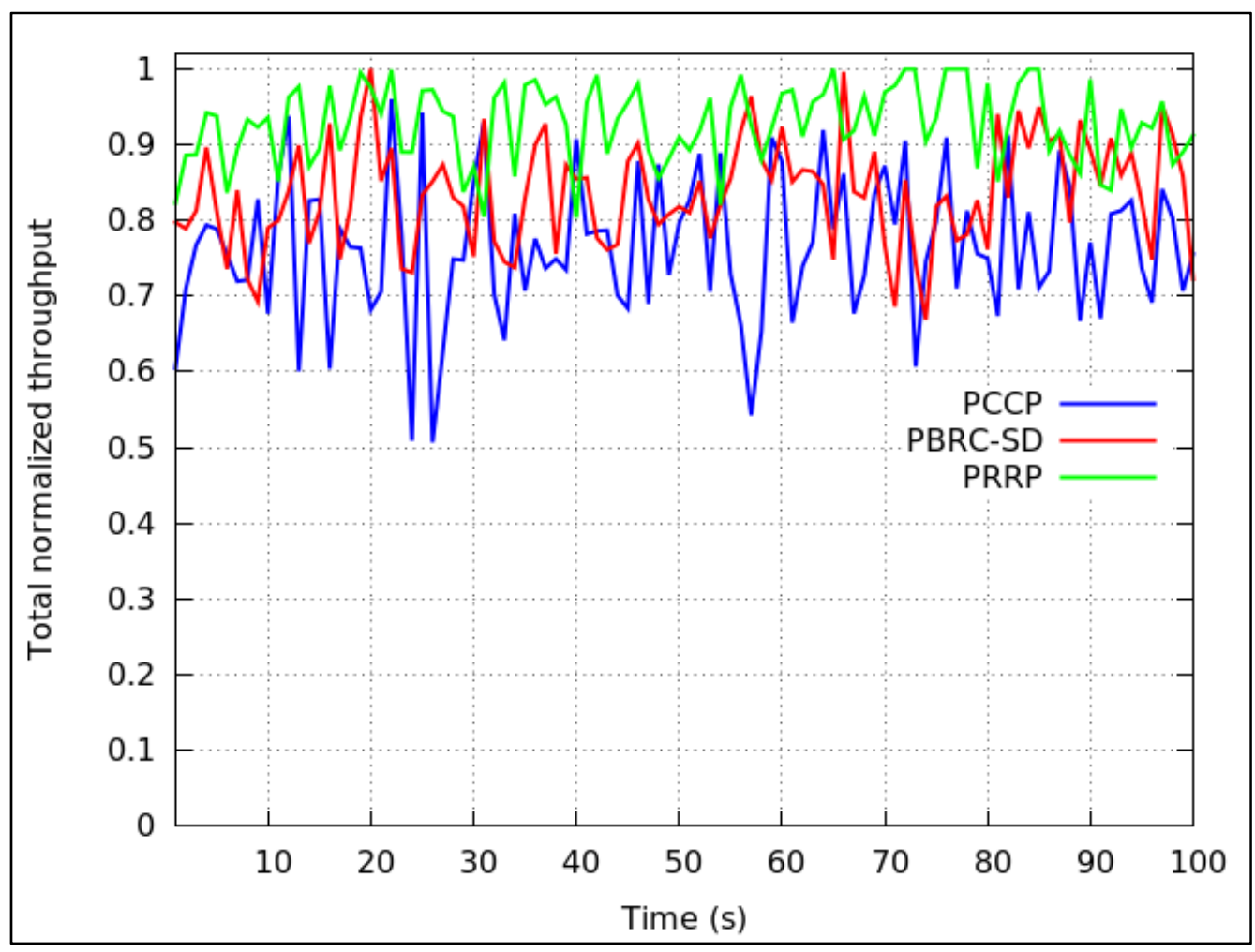

a) Normalized throughput

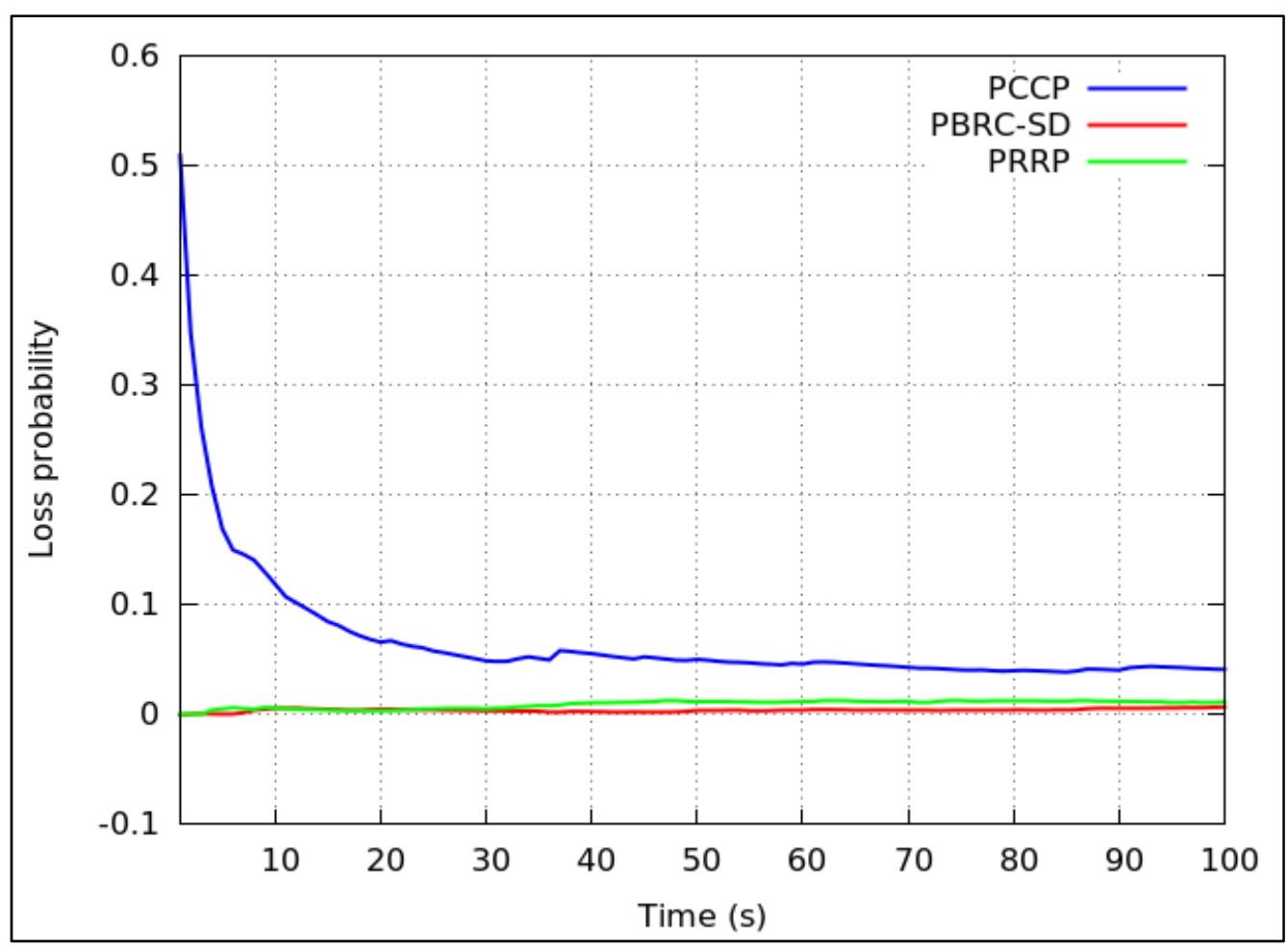

b) Loss probability

Figure 4.10: Results for random service time 


\section{Evaluating the WRR Scheduler}

\subsubsection{Performance of the Service Differentiation unit}

\subsubsection{Class-based results for PBRC-SD using the single-tier topology with extra parent node}

The WRR scheduler was used to service the different traffic classes' queues in both PRRP and PBRC-SD. The operation of WRR is discussed in Chapter 3 Section 3.2.1.3. In order to test the performance of the WRR scheduler, the source priority of Section 3.2 was assigned in such a way that the normalised weights for the four traffic classes are as follows: 0.5 for HPRT, 0.3 for HP-NRT, 0.15 for MP-NRT and 0.05 for LP-NRT. The WRR will serve each queue based on its weight. With these weights, the real-time multimedia traffic is guaranteed to receive more bandwidth from the sensor network than the other traffic classes.

Figure 4.11 presents the PBRC-SD results using random service time. The results show that the HP-RT class obtain the highest throughput, followed by the HP-NRT class. The LPNRT class has the lowest throughput. Achieving high throughput for HP-RT traffic means that real-time multimedia traffic of high importance, such as an alarm signal, gets the highest portion of the network resources thus enabling it to meet the QoS requirements of multimedia applications.

The queueing delay results of WRR scheduler in Figure $4.11 \mathrm{~b}$ ) also indicate that the HP-RT traffic has the lowest delay and MP-NRT has the highest delay. HP-RT traffic is expected to have the lowest queueing delay because it consists of real-time data which is delay sensitive. If HP-RT traffic encounters high queueing delay, the traffic will be useless at the receiver as it will be late to perform the initial reaction expected had it been that there was no delay in the network. All the traffic classes further experience zero packet loss. 


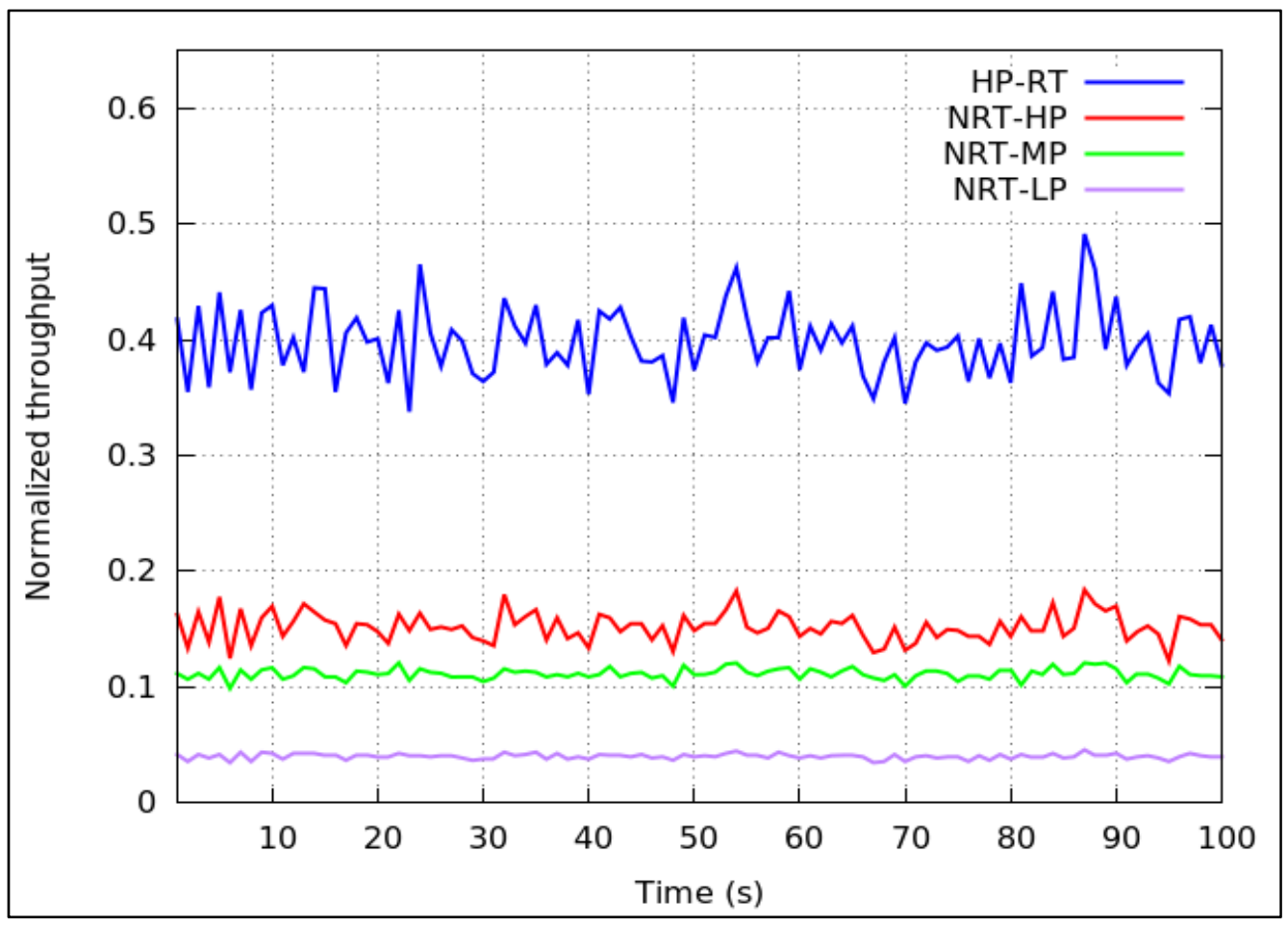

a) Normalized throughput

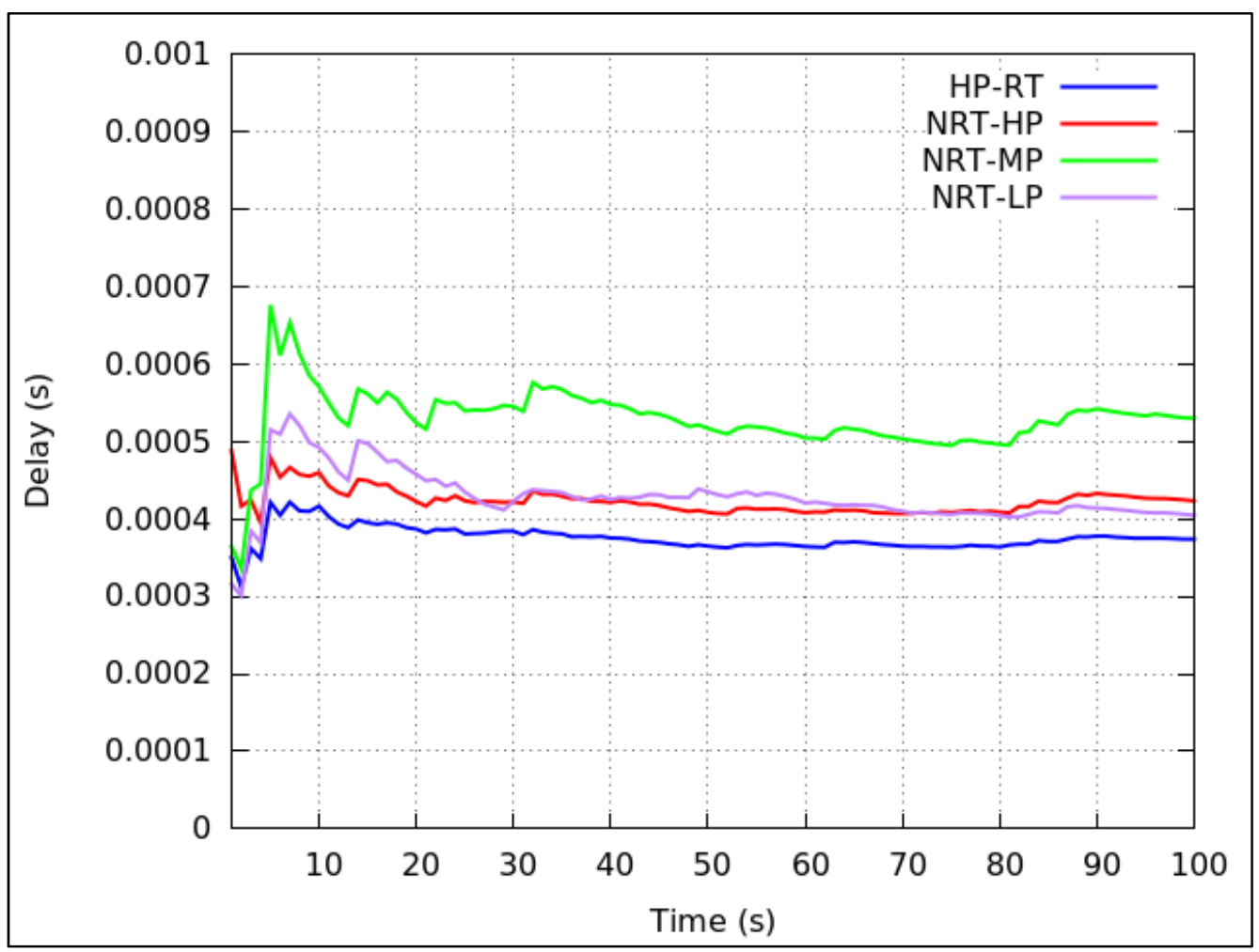

b) Delay (s)

Figure 4.11: Class based results for PBRC-SD 


\subsubsection{Class-based results for PRRP using the single-tier topology with extra parent node}

The normalized throughput and delay per class results for the PRRP protocol are presented in Figures 4.12 a) and 4.12 b) respectively. The results shown are those of random service time. In this scenario, the results for the single-tier topology with the extra parent node show that similar to PBRC-SD above, the HP-RT traffic class achieves the highest throughput and LP-NRT has the lowest throughput. The total throughput achieved by PCCP indicates that PCCP experiences high throughput; however, PCCP does not support service differentiation.

The delay per class also confirms that HP-RT traffic has the lowest delay as per the requirements of real-time traffic. Just as it can be seen in the previous section of PBRC-SD with extra parent node, the loss per class is also zero in all the traffic classes. Since PCCP only uses one buffer for all the traffic types, it experiences high queuing delaying [41].

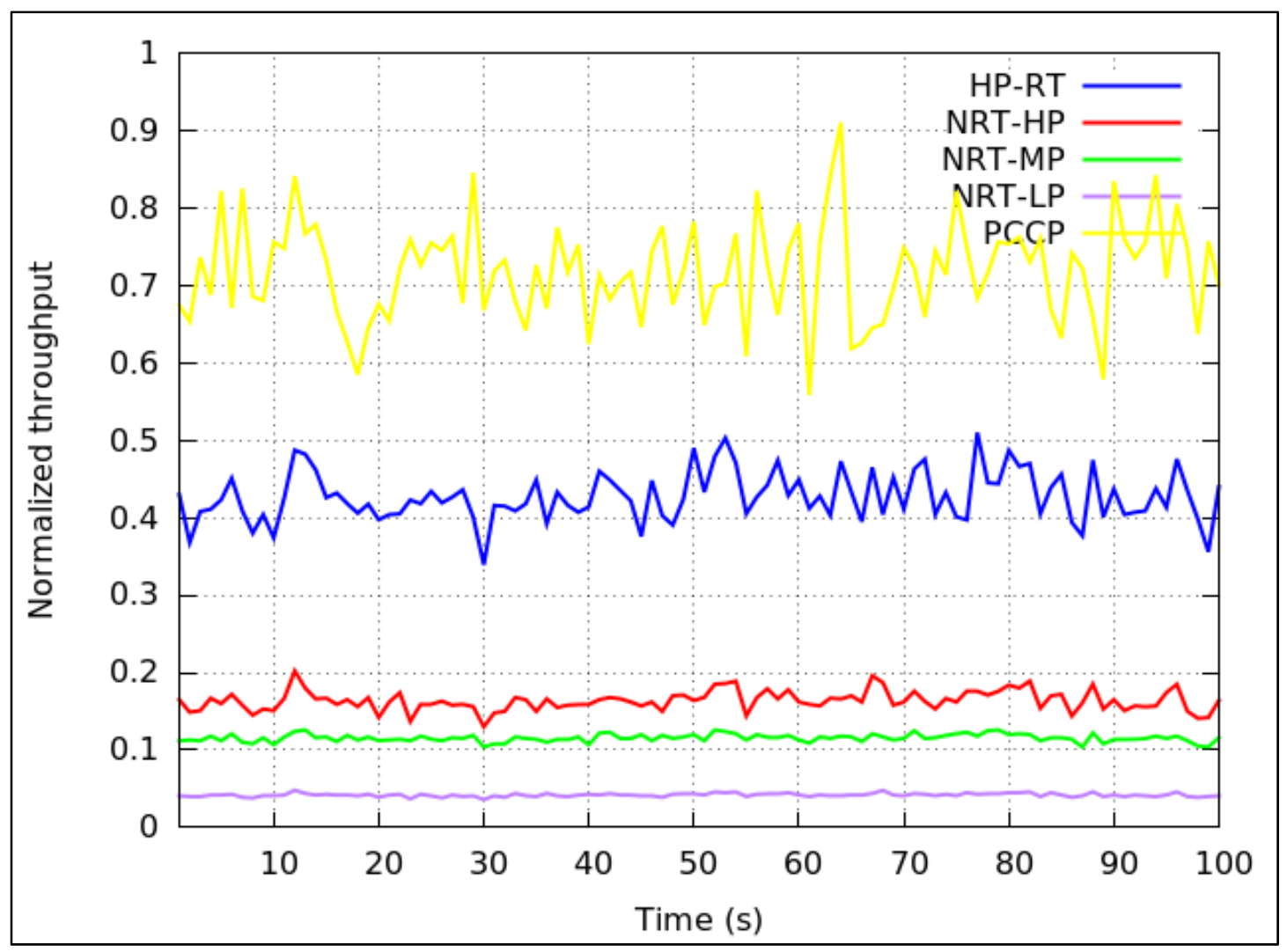

a) Normalized throughput 


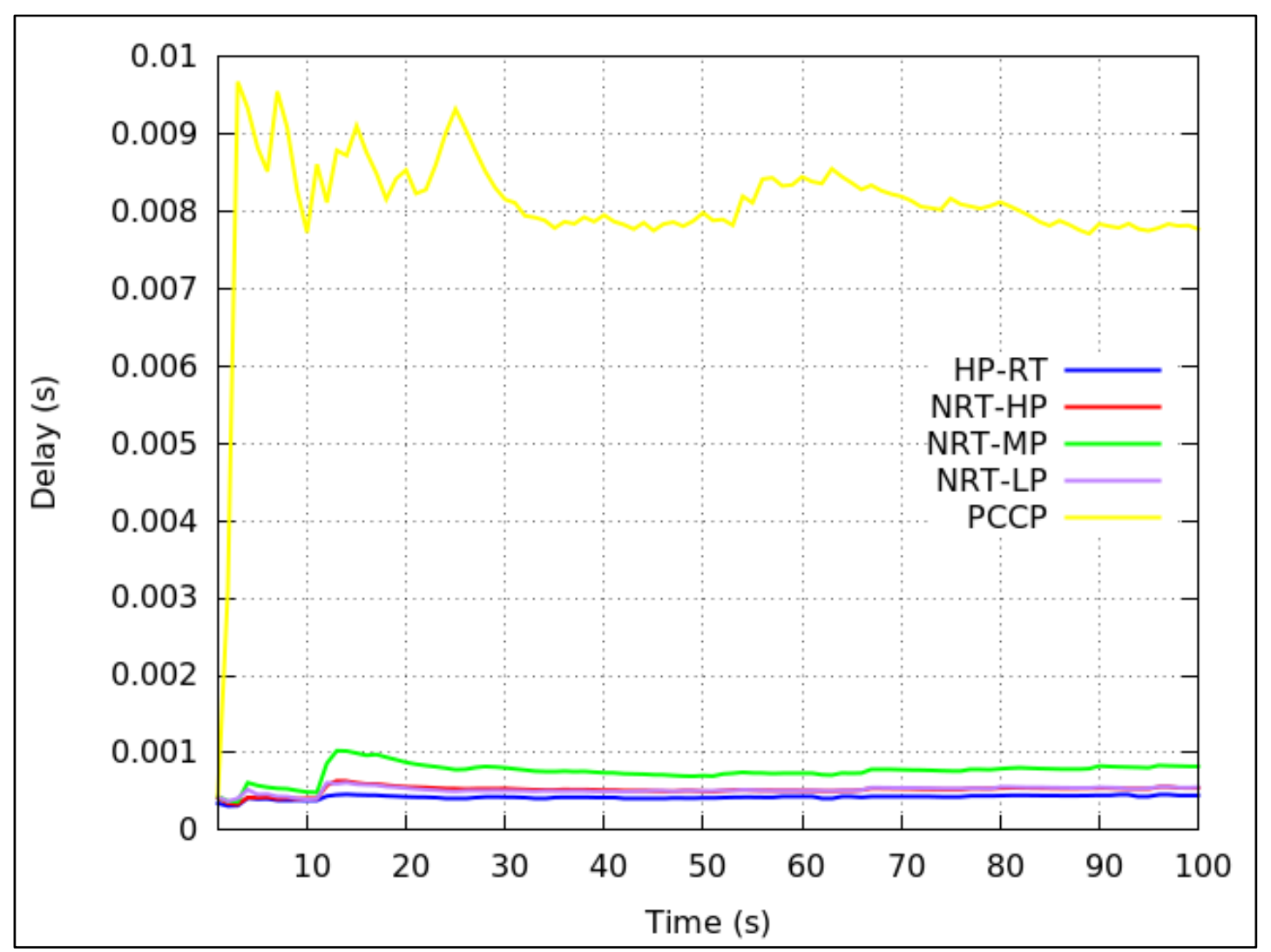

b) Delay (s)

Figure 4.12 Normalized throughput and delay (s) per class for PCCP and PRRP

Simulation results in this section show that the WRR scheduler used in PBRC-SD and PRRP is able to satisfy the needs of different classes having various service requirements.

\subsection{Energy results}

PRRP considers the energy of the upstream nodes when routing multimedia traffic. The consideration is made in order to extend the lifetime of the sensor network as transmitting nodes are not forced to always transmit to a dedicated upstream node. A number of selected nodes in the topology support multi-path routing which enables nodes to select between two available routes.

The results in Figure 4.13 show the energy consumption of individual nodes for PRRP, PBRC-SD and CCF when sensor nodes are transmitting CBR traffic service at fixed service time. The results for random service time are quite similar to that of CBR traffic. 


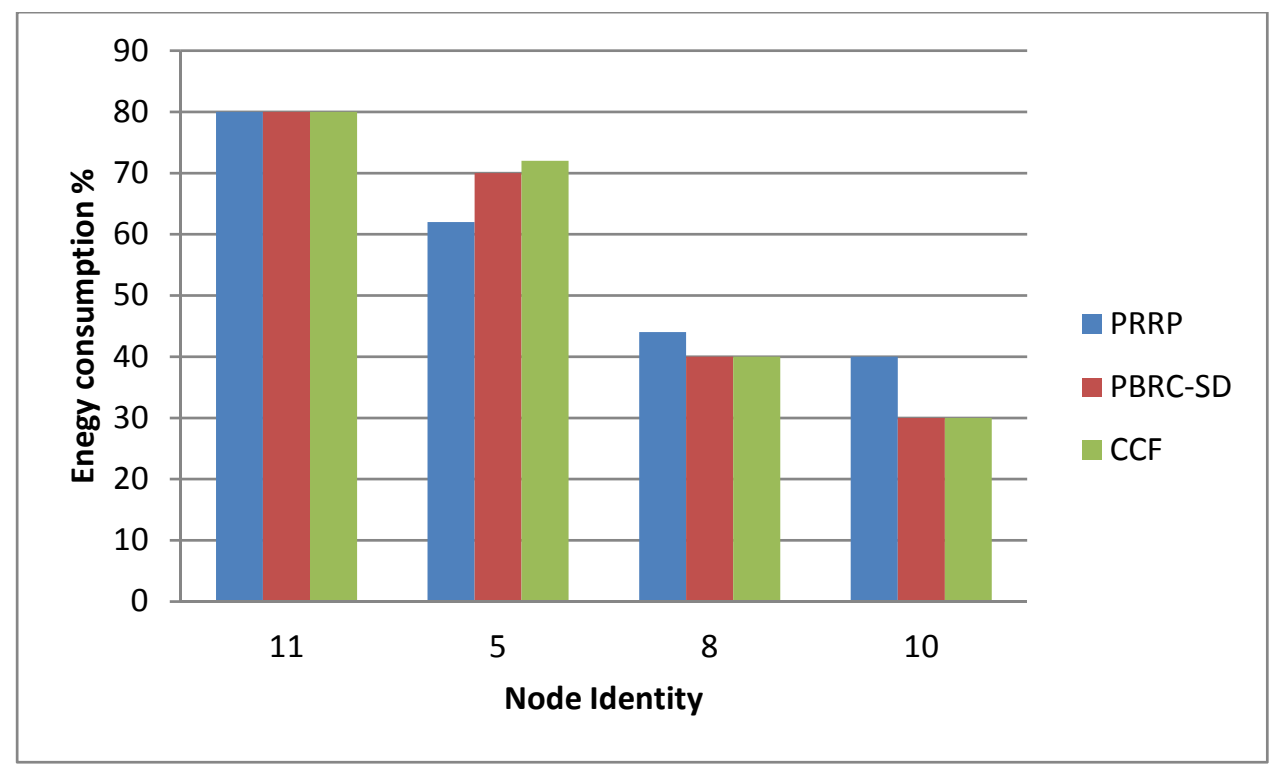

Figure 4.13 Energy consumption

From Figure 4.13, it is observed that for PRRP, PBRC-SD and CCF, the parent nodes closer to the sink consume more energy than any other parent nodes in the WSN. This is evident from the results of node 11, which is located closer to the sink, as shown in the multi-path topology in Figure 3.4. The high energy consumption at node 11 is a result of the combined traffic from the downstream sensor nodes transiting through it. The energy consumption of node 5 is also high because of the number of its child nodes. Node 8 and node 10 have the lowest energy consumption because they have few child nodes.

From the same figure, it is also observed that PRRP had better balance in its traffic and thus the energy consumed by nodes. For PRRP for example, the difference in the percentage of energy consumed between parent nodes 5 and 8 , or 8 and 10 is respectively 18 and 4, which is less significant when compared to PBRC-SD, where the difference is 30 and 20, or CCF 32 and 20 respectively. This is because in PRRP, a child node with two parents uses the energy level and the output rate of the parent node to decide which parent node to route its traffic through. Taking into account the energy level of nodes in particular leads to a better utilization of energy of nodes and more balance energy consumption among nodes. However, for both PBRC-SD and CCF a child node with two parents will keep using the same parent node to transmit its data; this leads to high utilization of its energy, which in turn may unbalance the energy among parent nodes creating a situation where part of the network will die while other parts are still active. 
The total residual energy of the three protocols in Figure 4.14 below shows that the PRRP obtains the highest residual energy when compared to PBRC-SD and CCF. This is because the PRRP protocol considers the energy of the nodes when routing traffic.

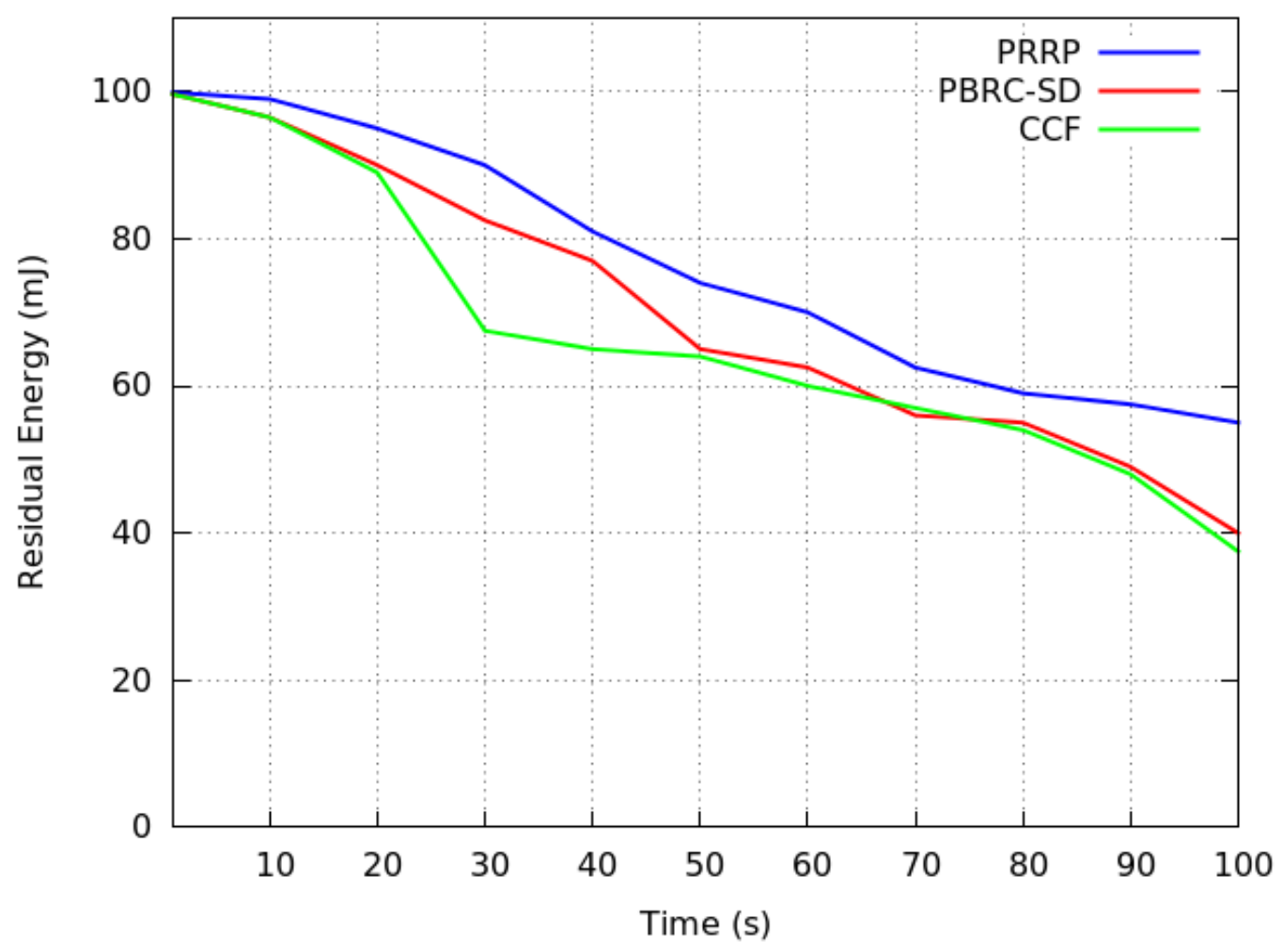

Figure 4.14 Residual Energy

\subsection{Discussion}

This chapter discussed the research findings that were obtained through simulations. The simulations started by first observing how PBRC-SD performs in terms of packet loss, queueing delay and throughput when using a single-tier with single-path topology, and then when one extra parent node is added to the single-tier topology and finally when multi-path routing topology is introduced.

Observations of PBRC-SD's performance indicate that it experienced a drop in throughput when an extra parent and/or multipath is introduced. The drop in throughput occurred due to a number of reasons. Firstly, the addition of the parent node increases the packets at the buffer causing the average queue size to be greater than the maximum threshold, leading to congestion. Secondly, the throughput has dropped due to the sensitivity of the traffic 
load to the RED parameter settings. Lastly, when multi-path is introduced, the rate adjustment algorithm cannot distribute the rates fairly, resulting in unbalanced traffic.

Further results analysis shows that the PBRC-SD queueing delay drops with the addition of the extra parent node and during multi-path routing. The change in topologies does not cause any further packet loss. Therefore, it can be concluded that PBRC-SD does not scale well in terms of queueing delay and throughput when additional nodes and/or multipath routing is added to the single-tier topology.

However, when the same simulations are performed for PRRP protocol, the following results were observed for random service time: PRRP shows a higher throughput than PBRCSD when the single-tier topology is modified with the addition of the extra parent node and/or multi-path routing. Similar to PBRC-SD, PRRP also reports zero packet loss probability; however, PRRP experiences some queueing delay.

Further useful results are that PRRP, like PBRC-SD, obtains class-dependent delays and throughput. For instance, the HP-RT traffic class achieves the lowest delay when compared with HP-NRT, MP-NRT and LP-NRT. This proves that PRRP meets the service requirements of multimedia as outlined in Chapter 2, Section 2.2.2.

Since PRRP also considers the energy of the nodes when routing traffic, simulation results showed that the PRRP is energy efficient when compared to PBRC-SD and CCF.

It can be concluded that PRRP achieved its objectives of improving PBRC-SD's performance, both when the extra parent nodes are added to the topology as well as when there are alternative routes in the topology. 


\subsection{Chapter Summary}

This chapter presented the simulation results carried out using the proposed PRRP on the proposed algorithm. Evaluations were performed using a single-tier network topology and a single-tier with multi-path routing network topology. Simulation results show that the throughput of PBRC-SD drops with an increase in the network nodes and when multi-path routing is used. This is attributed to the fixed threshold values used in the detection of queue length congestion. To overcome this problem, the PRRP algorithm that uses an adaptive threshold value to detect queue length congestion in the nodes, was introduced. The results in this chapter show that with this addition, PRRP improves the throughput of traffic classes. By using multi-path routing, the WMSNs is able to save the energy of the nodes thereby extending the lifetime of the sensor network. 


\section{CHAPTER 5}

\section{CONCLUSION AND FUTURE WORK}

\subsection{Introduction}

This chapter provides a summary of what has been discussed in the previous chapters, as well as providing the future work of the research.

\subsection{Summary of this research}

WMSNs consist of data with varying levels of importance that have strict requirements and often need to reach the sink within a predefined time. However, due to the presence of node level and link level congestion, buffer overflow is likely to occur that can have multiple effects on the data being communicated. Existing techniques that can combat congestion in WMSNs can be classified as either traffic control protocols, resource control protocols or a hybrid of both protocols. Since congestion is a temporary event, resource control techniques are inefficient in WMSNs as they may waste resources. Therefore, traffic control techniques are preferred as they are concerned with controlling traffic flow in the network through adjusting the transmission rates. Literature of the existing traffic control protocols were discussed together with the drawbacks of each protocol in Chapter 2.

In this research, we addressed service differentiation and congestion issues in wireless multimedia sensor networks by proposing a Priority Rate-based Routing Protocol (PRRP). for Wireless Multimedia Sensor Network. PRRP solves these issues by designing a node model which classifies traffic into different traffic classes; each traffic class accommodates different multimedia traffic and scalar data depending on their service requirements. Since real-time multimedia traffic has strict service requirements, it is assigned high priority which receives the highest weights to ensure that these service requirements are met.

Using this service differentiation model, the PRRP protocol follows three stages in controlling congestion, namely congestion detection, congestion notification and rate adjustment. One of the contributions highlighted by this research lies in the congestion detection stage, where an Adaptive Random Early Detection (A-RED) is used to detect the presence of congestion in the node while dynamically adjusting the threshold parameters to meet the changes in the WSN conditions. 
In order to test the effectiveness of PRRP, simulations were performed using both single-path routing and multi-path routing. Another contribution made during this research was to test the performance of PRRP on a multi-path topology. This is because most of the existing priority-based rate protocols were only evaluated using single-path routing, which does not consider the energy of the nodes. Since sensor nodes networks are characterised as energy constrained, our PRRP supports multi-path routing, in which the energy of the nodes is considered before transmitting traffic to upstream nodes.

Evaluations of PRRP have demonstrated through simulations that this protocol is able to achieve higher throughput, low packet loss and low queueing delay when compared with PCCP and PBRC-SD. Further evaluations show that PRRP can allocate priority-dependant QoS to the various traffic thus guaranteeing real-time multimedia traffic the strict service requirements. By supporting multi-path routing with energy consideration, the PRRP shows that it has obtained lower energy consumptions than PBRC-SD and CCF.

\subsection{Recommendations for Future work}

1. Investigate other QoS parameters: This research only focused on obtaining the packet delay, throughput and packet loss of PRRP. Other future work can extend these parameters to evaluate the algorithm in terms of fairness and reliability.

2. Introduce more alternative routes in the topology: Currently, only two multipath routes are considered in the topology used in the simulation. It will be interesting to see how PRRP performs with the addition of more alternative routes in the topology while still ensuring that the redundant multimedia traffic generated by the nodes does not deplete the energy of the sensor nodes at the same time.

3. Detect congestion based on multimedia characteristics: Although the rate based congestion control techniques in WMSNs are able to meet the QoS requirements of multimedia data, they may compromise the quality of the data. For this reason, the characteristics of the multimedia data can be considered during congestion control so that all the multimedia data frames are not equally affected by the change in transmission rates. 


\section{References}

[1] J. Yick, B. Mukherjee, and D. Ghosal, "Wireless sensor network survey," Comput. Networks, vol. 52, no. 12, pp. 2292-2330, 2008.

[2] A. Sharif, V. Potdar, and E. Chang, "Wireless multimedia sensor network technology: A survey," 2009 7th IEEE Int. Conf. Ind. Informatics, pp. 606-613, 2009.

[3] T. Znati and S. Krishna, "Wireless Sensor Network," 2004th ed., Kluwer Academic Publishers, 2004, pp. 52, 265.

[4] K. Sohraby, Wireless Sensor Networks:Technology, Protocols and Applications. New Jersey: John Wiley \& Sons, 2007.

[5] I. F. Akyildiz, T. Melodia, and K. R. Chowdhury, "Wireless Multimedia Sensor Networks: Applications and Testbeds," Proc. IEEE, vol. 96, no. 10, pp. 1588-1605, 2008.

[6] I. F. Akyildiz, T. Melodia, and K. R. Chowdhury, "A survey on wireless multimedia sensor networks," Comput. Networks, vol. 51, no. 4, pp. 921-960, Mar. 2007.

[7] S. Ehsan and B. Hamdaoui, "A survey on energy-efficient routing techniques with QoS assurances for wireless multimedia sensor networks," IEEE Commun. Surv. Tutorials, vol. 14, no. 2, pp. 265-278, 2012.

[8] S. Misra, M. Reisslein, and G. L. Xue, "A Survey of Multimedia Streaming in Wireless Sensor Networks," leee Commun. Surv. Tutorials, vol. 10, no. 4, pp. 18-39, 2008.

[9] A. Tanenbaum, "Summary for Policymakers," in Climate Change 2013 - The Physical Science Basis, Fourth Edi., Intergovernmental Panel on Climate Change, Ed. Cambridge: Cambridge University Press, 2003, pp. 1-30.

[10] A. Leon-Garcia and I. Widjaja, "Communication Networks," McGraw-Hill, 2003, pp. 516-517.

[11] M. O. Farooq, T. Kunz, and M. St-Hilaire, "Differentiated services based congestion control algorithm for Wireless Multimedia Sensor Networks," in 2011 IFIP Wireless Days (WD), 2011, pp. 1-6.

[12] P. Antoniou, A. Pitsillides, T. Blackwell, A. Engelbrecht, and L. Michael, "Congestion control in wireless sensor networks based on bird flocking behavior," Comput. Networks, vol. 57, no. 5, pp. 1167-1191, 2013.

[13] S. Mahdizadeh Aghdam, M. Khansari, H. R. Rabiee, and M. Salehi, "WCCP: A congestion control protocol for wireless multimedia communication in sensor networks," Ad Hoc Networks, vol. 13, no. PART B, pp. 516-534, 2014.

[14] J. Kang, Y. Zhang, and B. Nath, "TARA: Topology-aware resource adaptation to alleviate congestion in sensor networks," IEEE Trans. Parallel Distrib. Syst., vol. 18, no. 7, pp. 919-931, 2007.

[15] C. Sergiou, V. Vassiliou, and A. Paphitis, "Hierarchical Tree Alternative Path (HTAP) algorithm for congestion control in wireless sensor networks," Ad Hoc Networks, vol. 11, no. 1, pp. 257-272, 2013. 
[16] C.-Y. Wan, S. B. Eisenman, and A. T. Campbell, "Energy-efficient congestion detection and avoidance in sensor networks," ACM Trans. Sens. Networks, vol. 7, no. 4, pp. 1-31, 2011.

[17] E. Gisirses, "Multimedia communication in wireless sensor networks," Ann. Des Télécommunications, vol. 60, no. 7, pp. 872-900, 2005.

[18] I. Politis, M. Tsagkaropoulos, and S. Kotsopoulos, "Optimizing Video Transmission over Wireless Multimedia Sensor Networks," pp. 1-6, 2008.

[19] S. Paniga, L. Borsani, A. Redondi, M. Tagliasacchi, M. Cesana, P. Milano, and P. Leonardo, "Experimental Evaluation of a Video Streaming System for Wireless Multimedia Sensor Networks," pp. 165-170, 2011.

[20] S. Guo and T. D. C. Little, "QoS-enabled Video Streaming in Wireless Sensor Networks," in 2010 Ninth IEEE International Symposium on Network Computing and Applications, 2010, pp. 214-217.

[21] J. F. Kurose and K. W. Ross, "Computer Networking: A Top-Down Approach," 6th ed., Pearson Education, 2012, pp. 35-38.

[22] M. C. Domingo, "Marine communities based congestion control in underwater wireless sensor networks," Inf. Sci. (Ny)., vol. 228, pp. 203-221, 2013.

[23] M. Bramberger, J. Brunner, B. Rinner, and H. Schwabach, "Real-time video analysis on an embedded smart camera for traffic surveillance," Proceedings. RTAS 2004. 10th IEEE Real-Time Embed. Technol. Appl. Symp. 2004., pp. 174-181, 2004.

[24] C. Sonmez, O. Incel, S. Isik, M. Donmez, and C. Ersoy, "Fuzzy-based congestion control for wireless multimedia sensor networks," EURASIPJ. Wirel. Commun. Netw., vol. 2014, no. 1, p. 63, 2014.

[25] K. Akkaya and M. Younis, "A survey on routing protocols for wireless sensor networks," Ad Hoc Networks, vol. 3, no. 3, pp. 325-349, 2005.

[26] Jayakumari, "Priority Based Congestion Detection and Avoidance in Wireless Sensor Networks," J. Comput. Sci., vol. 9, no. 3, pp. 350-357, 2013.

[27] Q. Lin, R. Wang, J. Guo, and L. Sun, "Novel congestion control approach in wireless multimedia sensor networks," J. China Univ. Posts Telecommun., vol. 18, no. 2, pp. 18, 2011.

[28] P. Kulkarni, D. Ganesan, P. Shenoy, and Q. Lu, "The case for multi--tier camera sensor networks," Proc. Int. Work. Netw. Oper. Syst. Support Digit. audio video - NOSSDAV'05, p. 141, 2005.

[29] J. Campbell, P. Gibbons, S. Nath, P. Pillai, S. Seshan, and R. Sukthankar, "IrisNet: an internet-scale architecture for multimedia sensors," Proc. 13th Annu. ACM Int. Conf. Multimed., pp. 81-88, 2005.

[30] S. Hengstler, D. Prashanth, S. F. S. Fong, and H. Aghajan, "MeshEye: A HybridResolution Smart Camera Mote for Applications in Distributed Intelligent Surveillance," 2007 6th Int. Symp. Inf. Process. Sens. Networks, pp. 360-369, 2007.

[31] S. Zacharias and T. Newe, "Technologies and Architectures for Multimedia-Support in 
Wireless Sensor Networks," NTECH Open Access, 2010, pp. 374-380.

[32] P. Rawat, K. D. Singh, H. Chaouchi, and J. M. Bonnin, "Wireless sensor networks: a survey on recent developments and potential synergies," J. Supercomput., vol. 66, no. 1, p. 48 pages, 2013.

[33] M. Al Nuaimi, F. Sallabi, and K. Shuaib, "A survey of Wireless Multimedia Sensor Networks challenges and solutions," 2011 Int. Conf. Innov. Inf. Technol., pp. 191-196, 2011.

[34] A. A. Abbasi and M. Younis, "A survey on clustering algorithms for wireless sensor networks," Comput. Commun., vol. 30, no. 14-15, pp. 2826-2841, 2007.

[35] A. Mainwaring, D. Culler, J. Polastre, R. Szewczyk, and J. Anderson, "Wireless Sensor Networks for Habitat Monitoring," Proc. 1st \{ACM\} Int. Work. Wirel. Sens. Networks Appl., pp. 88-97, 2002.

[36] B. Sharma and T. C. Aseri, "A Comparative Analysis of Reliable and Congestion-Aware Transport Layer Protocols for Wireless Sensor Networks," ISRN Sens. Networks, vol. 2012, pp. 1-14, 2012.

[37] H. Yuan, N. Yugang, and G. Fenghao, "Congestion control for wireless sensor networks: A survey," in The 26th Chinese Control and Decision Conference (2014 CCDC), 2014, pp. 4853-4858.

[38] C. Wang, B. Li, K. Sohraby, M. Daneshmand, and Y. Hu, "Upstream congestion control in wireless sensor networks through cross-layer optimization," IEEE J. Sel. Areas Commun., vol. 25, no. 4, pp. 786-795, May 2007.

[39] C. Wang, B. Li, K. Sohraby, M. Daneshmand, and Y. Hu, "Upstream congestion control in wireless sensor networks through cross-layer optimization," Sel. Areas Commun. IEEE J., vol. 25, no. 4, pp. 786-795, 2007.

[40] C. T. Ee and R. Bajcsy, "Congestion control and fairness for many-to-one routing in sensor networks," in Proceedings of the 2nd international conference on Embedded networked sensor systems, 2004, pp. 148-161.

[41] M. H. Yaghmaee and D. a. Adjeroh, "Priority-based rate control for service differentiation and congestion control in wireless multimedia sensor networks," Comput. Networks, vol. 53, no. 11, pp. 1798-1811, 2009.

[42] B. Hull, K. Jamieson, and H. Balakrishnan, "Mitigating congestion in wireless sensor networks," in Proceedings of the 2nd international conference on Embedded networked sensor systems - SenSys '04, 2004, p. 134.

[43] C.-Y. Wan, S. B. Eisenman, A. T. Campbell, and J. Crowcroft, "Siphon: Overload Traffic Management using Multi-Radio Virtual Sinks in Sensor Networks," Proc. 3rd Int. Conf. Embed. networked Sens. Syst. - SenSys '05, vol. 3, p. 116, 2005.

[44] M. Monowar, O. Rahman, A. Pathan, and C. Hong, "Prioritized heterogeneous trafficoriented congestion control protocol for WSNs," Int. Arab J. Inf. Technol., vol. 9, no. 1, pp. 39-48, 2012.

[45] M. Yaghmaee and D. Adjeroh, "A New Priority Based Congestion Control Protocol for 
Wireless Multimedia Sensor Networks," Int. Symp. a World Wireless, Mob. Multimed. Networks, 2008. WoWMoM 2008, pp. 1-8, 2008.

[46] S. Floyd and V. Jacobson, "Random Early Detection Gateways for Congestion Avoidance," IEEE/ACM Trans. Netw., vol. 1, no. 4, pp. 397-413, 1993.

[47] R. Gummadi, "Adaptive RED : An Algorithm for Increasing the Robustness of RED ' $s$ Active Queue Management," University of California at Berkeley, 2002.

[48] S. Floyd, R. Gummadi, and S. Shenker, "Adaptive RED: An algorithm for increasing the robustness of RED's active queue management," 2001.

[49] X. Chang, "Network simulations with OPNET," in Proceedings of the 31st conference on Winter simulation Simulation---a bridge to the future - WSC'99, 1999, vol. 1, pp. 307314.

[50] D. Verkest, J. Kunkel, and F. Schirrmeister, "System level design using C++," in Proceedings of the conference on Design, automation and test in Europe - DATE '00, 2000, pp. 74-83.

[51] "The Network Simulator ns-2: Documentation." [Online]. Available: http://www.isi.edu/nsnam/ns/ns-documentation.html. [Accessed: 25-Jul-2016].

[52] T. R. Henderson and G. F. Riley, "Network Simulations with the ns-3 Simulator," in SIGCOMM demonstration, 2008, p. 17.

[53] A. Varga and R. Hornig, "AN OVERVIEW OF THE OMNeT ++ SIMULATION ENVIRONMENT," in Proceedings of the 1st international conference on Simulation tools and techniques for communications, networks and systems \& workshops, 2008, p. 60.

[54] J. Farooq and T. Turletti, "An IEEE 802.16 WiMAX module for the NS-3 simulator," in Proceedings of the Second International ICST Conference on Simulation Tools and Techniques, 2009, pp. 415-422.

[55] G. Piro, D. E. E. Politecnico, N. Baldo, and M. Miozzo, "An LTE module for the ns-3 network simulator," pp. 415-422, 2011.

[56] H. Wu, S. Nabar, and R. Poovendran, "An Energy Framework for the Network Simulator 3 (ns-3)," in Proceedings of the 4th International ICST Conference on Simulation Tools and Techniques, 2011, vol. 3, pp. 222-230.

[57] M. May, J. Bolot, C. Diot, and B. Lyles, "Reasons not to deploy RED," 1999 Seventh Int. Work. Qual. Serv. IWQoS'99. (Cat. No.98EX354), pp. 0-2, 1999. 


\section{Appendix A: Simulation Results}

\section{A.1 PBRC-SD Results using RED}

Constant Bit Rate traffic and Fixed service time

Table A.1

\begin{tabular}{|c|c|c|c|}
\hline \multicolumn{4}{|c|}{ Normalized throughput } \\
\hline & PCCP & CCF & PBRC-SD \\
\hline Single-tier topology & 0.968 & 0.999 & 0.98 \\
\hline Single-tier with extra parent & 0.947 & 0.893 & 0.799 \\
\hline Multi-path topology & 0.848 & 0.905 & 0.783 \\
\hline
\end{tabular}

Table A.2

\begin{tabular}{|l|r|r|r|}
\hline \multicolumn{4}{|c|}{ Delay (s) } \\
\hline & \multicolumn{1}{|c|}{ PCCP } & \multicolumn{1}{c|}{ CCF } & \multicolumn{1}{c|}{ PBRC-SD } \\
\hline Single-tier topology & 0.0005 & 0.001 & 0.0007 \\
\hline Single-tier with extra parent & 0.0005 & 0.00034 & 0.000338 \\
\hline Multi-path topology & 0.000408 & 0.000302 & 0.00022 \\
\hline
\end{tabular}

\section{Exponential traffic and Random service time}

Table A.3

\begin{tabular}{|l|r|r|r|}
\hline \multicolumn{4}{|c|}{ Normalized throughput } \\
\hline & PCCP & \multicolumn{1}{|c|}{ CCF } & \multicolumn{1}{c|}{ PBRC-SD } \\
\hline Single-tier topology & 0.826 & 0.999 & 0.931 \\
\hline Single-tier with extra parent & 0.686 & 0.929 & 0.824 \\
\hline Multi-path topology & 0.765 & 0.948 & 0.835 \\
\hline
\end{tabular}


Table A.4

\begin{tabular}{|l|r|r|r|}
\hline \multicolumn{4}{|c|}{ Loss probability } \\
\hline & \multicolumn{1}{|c|}{ PCCP } & \multicolumn{1}{c|}{ CCF } & \multicolumn{1}{|c|}{ PBRC } \\
\hline Single-tier topology & 0.025 & 0.065 & 0.0005 \\
\hline Single-tier with extra parent & 0.001 & 0 & 0 \\
\hline Multi-path topology & 0.000078 & 0 & 0.00487 \\
\hline
\end{tabular}

Table A.5

\begin{tabular}{|l|r|r|r|}
\hline \multicolumn{3}{|c|}{ Delay (s) } \\
\hline & PCCP & \multicolumn{1}{c|}{ CCF } & PBRC-CD \\
\hline Single-tier topology & 0.021 & 0.081 & 0.009 \\
\hline Single-tier with extra parent & 0.0087 & 0.0053 & 0.0019 \\
\hline Multi-path topology & 0.0045 & 0.019 & 0.01 \\
\hline
\end{tabular}

\section{A.2 PRRP Results using A-RED}

$\underline{\text { Exponential traffic and Random Service time results }}$

Table A.6

\begin{tabular}{|l|r|r|r|}
\hline \multicolumn{4}{|c|}{ Normalized Throughput } \\
\hline & PCCP & \multicolumn{1}{|c|}{ PBRC-SD } & PRRP \\
\hline Single-tier topology & 0.826 & 0.931 & 0.996 \\
\hline Single-tier with extra parent & 0.686 & 0.824 & 0.885 \\
\hline Multi-path topology & 0.765 & 0.835 & 0.929 \\
\hline
\end{tabular}


Table A. 7

\begin{tabular}{|l|r|r|r|}
\hline \multicolumn{3}{|c|}{ Loss } & \\
\hline & \multicolumn{1}{|c|}{ PCCP } & \multicolumn{1}{l|}{ PBRC-SD } & PRRP \\
\hline Single-tier topology & 0.025 & 0.0005 & 0.00878 \\
\hline Single-tier with extra parent & 0.01 & 0 & 0 \\
\hline Multi-path topology & 0.00078 & 0.00487 & 0.000736 \\
\hline
\end{tabular}

Table A.8

\begin{tabular}{|l|r|r|r|}
\hline \multicolumn{3}{|c|}{ Delay (s) } \\
\hline & \multicolumn{1}{|c|}{ PCCP } & \multicolumn{1}{|c|}{ PBRC-SD } & \multicolumn{1}{|c|}{ PRRP } \\
\hline Single-tier topology & 0.021 & 0.009 & 0.0776 \\
\hline Single-tier with extra parent & 0.00875 & 0.0019 & 0.004194 \\
\hline Multi-path topology & 0.00455 & 0.001 & 0.01463 \\
\hline
\end{tabular}

